UNITED STATES DEPARTMENT OF THE INTERIOR GEOLOGICAL SURVEY

GEOPHYSICAL LOGS FOR DAWSON, GARFIELD, MCCONE, AND PRAIRIE COUNTIES, MONTANA

\author{
Chapter D of \\ Preliminary Report of 1977 Coal Drilling in \\ Eastern Montana and Northeastern Wyoming
}

By U.S. Geological Survey

and

Montana Bureau of Mines and Geology

Open-File Report 77-721-D

1978

This report has not been edited

for conformity with Geological

Survey editorial standards or

stratigraphic nomenclature. 


\section{Contents}

Page

Introduction and acknowledgments. . . . . . . . . D D1

Geophysical logs. . . . . . . . . . . . . $\mathrm{D} 2$

References. . . . . . . . . . . . . . . . D100

\section{EXPLANATION OF DRILL-HOLE LOCATIONS}

The location of a drill hole within a section is identified on the logs by a letter-designated tract system and by footage from section lines. Each section is subdivided into four quadrants, with the northeast quadrant designated as $\mathrm{A}$, and continuing counterclockwise to $\mathrm{D}$. Each quadrant is then subdivided into four equal parts with a similar letter designation. This is repeated until a section is subdivided into 256 units. Using this system, the largest subdivision in a section is listed first. For example, a tract designation of $A B C D$ would be located in the $\mathrm{SE}^{\frac{1}{4}} \mathrm{SW}_{\frac{1}{4}} \mathrm{NW}^{\frac{1}{4}} \mathrm{NE} \frac{1}{4}$ of the section. 
PRELIMINARY REPORT OF 1977 COAL DRILLING IN EASTERN MONTANA AND NORTHEASTERN WYOMING

\author{
GEOPHYSICAL LOGS FOR DAWSON, GARFIELD, MCCONE, AND PRAIRIE \\ COUNTIES, MONTANA
}

\title{
INTRODUCTION AND ACKNOWLEDGMENTS
}

This report is the fourth in a series on the 1977 drilling program (hole nos. 77149-77199) being conducted by the Montana Bureau of Mines and Geology (MBMG) under Grant No. 14-08-0001-G-441 from the U. S. Geological Survey (USGS). Other chapters will follow as data are received. The drilling is being done as part of a USGS program to evaluate and classify mineral lands in the public domain. Similar information from 856 drill holes and approximately 200 coal samples from the same areas and from western North Dakota was presented in earlier reports (USGS/ MBMG 1973, 1974, 1976a, 1976b, 1977a, 1977b, 1977c, 1978; USGS/NDGS . 1976, 1977; and Kistner, 1977).

This report includes only geophysical logs. Core samples from some of the holes have been submitted to the U. S. Bureau of Mines for proximate analyses, determination of $\mathrm{Btu} / \mathrm{lb}$ values, and form of sulfur present. Fieldwork was carried out by the following MBMG personnel: Robert E. Matson, Chief, Energy Division; Miller Hansen and John Pinchock, geologists; and Leigh Sauer and J. Jensen, technicians. Herbert Wincentsen, USGS geologist, was also involved in the fieldwork. Coordination and technical guidance were provided by Robert E. Matson, MBMG, and Elmer M. Sche11, USGS. 
MONTANA BUREAU OF MINES AND GEOLOGY

Montana College of Mineral Science and Technology

Hole no: US-77149 Map: BLM IE 39

Date 9/11/77State: Montana County: Dawson

Elev. $2868 \mathrm{ft}$ Location: T._15 N.R. $52 \mathrm{E}, \mathrm{Sec} .26$, Tract BCCC Drilled depth: $300 \mathrm{ft}$

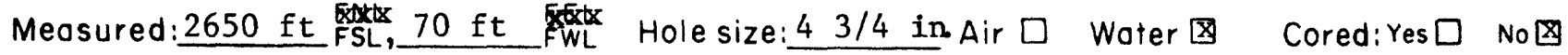
Remarks :

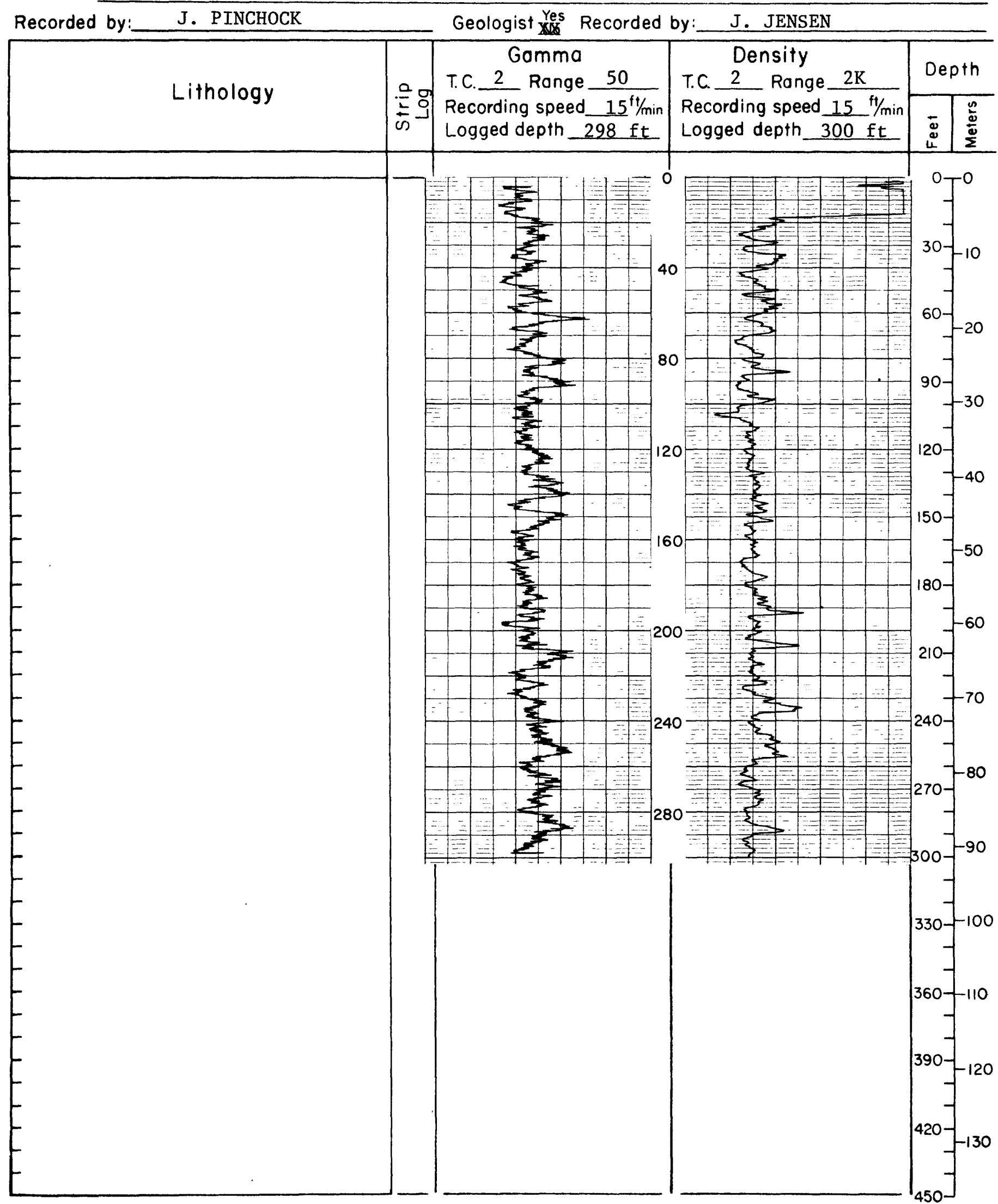


MONTANA BUREAU OF MINES AND GEOLOGY

Montana College of Mineral Science and Technology

Hole no: US-77150 Mop:BJM-NE 39

Date: $9 / 11 / 7$ Btate: Montana County: Dawson

Elev.:2692 ft Location: T. 15 N. R. 52 E. Sec. 24 , Tract DAAA Drilled depth: $420 \mathrm{ft}$

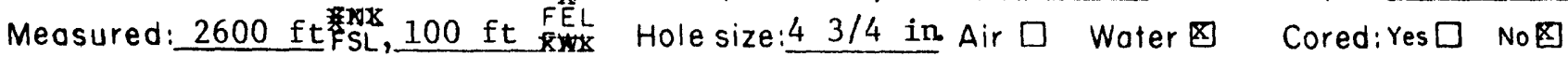

Remorks:

Recorded by:_J. PINCHOCK

Lithology

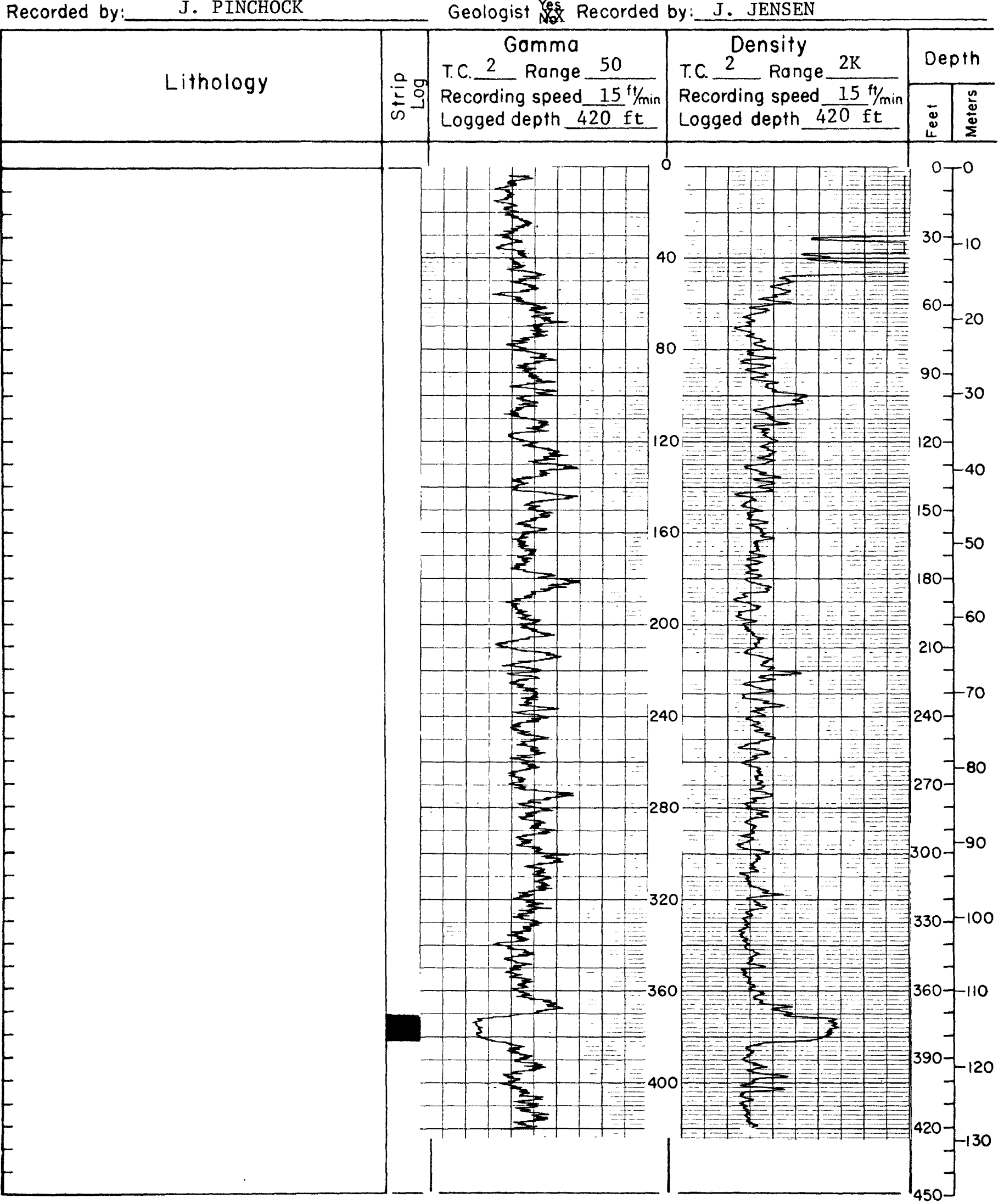


Hole number (continued)

$\underline{\text { US }-77150}$

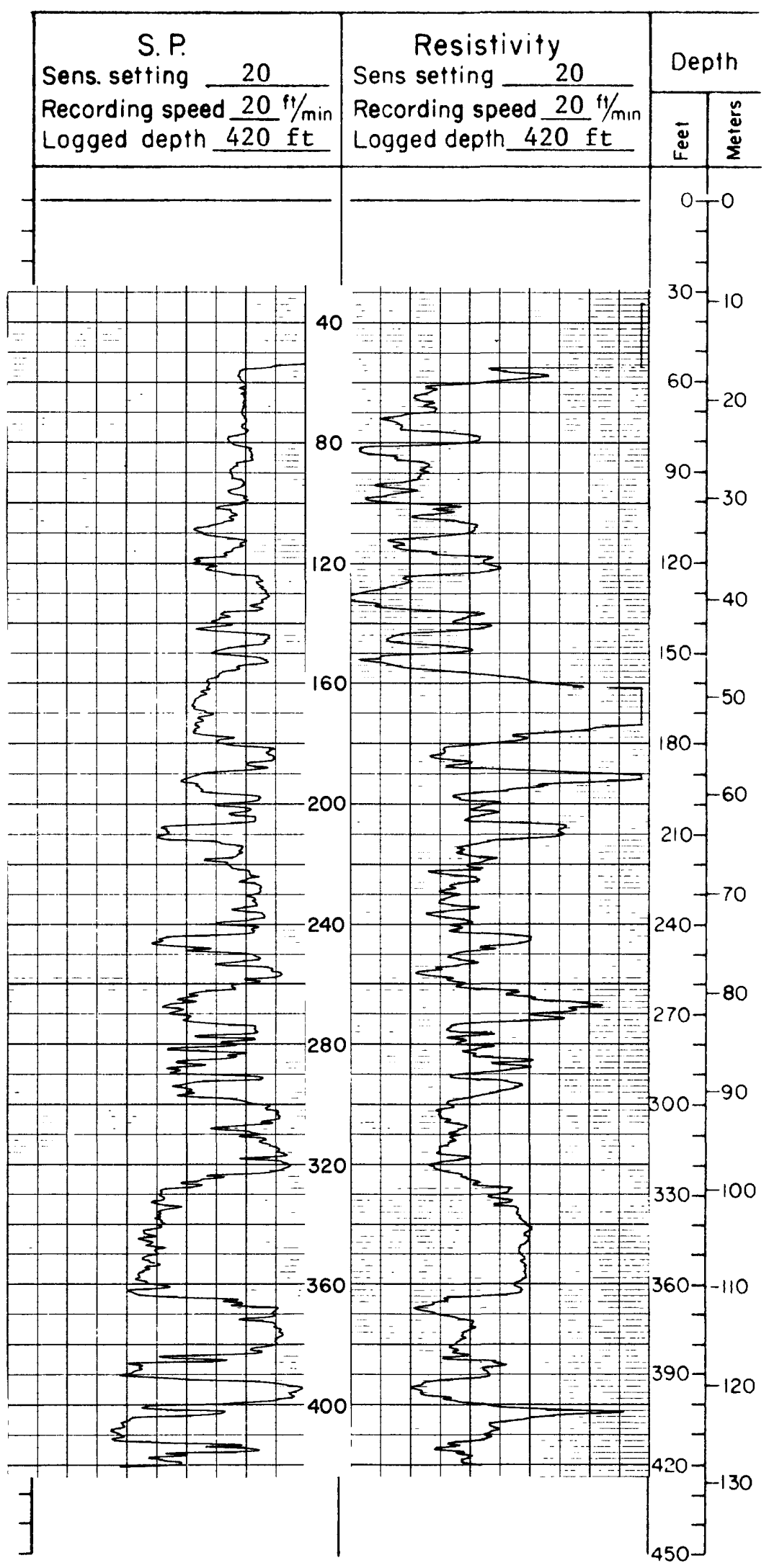




\section{MONTANA BUREAU OF MINES AND GEOLOGY \\ Montana College of Mineral Science and Technology}

Hole no: US-77151 Map: BLM-NE 39

Dote:8/12/775tate: Montana County: Dawson

Elev. $2898 \mathrm{ft}$ Location: T. $15 \mathrm{~N}, \mathrm{R} .52 \mathrm{E}$, Sec. 14 , Troct CBCC Drilled depth: $400 \mathrm{ft}$ Meosured:1330 ftFSL, $25 \mathrm{ft} \quad \mathrm{FWX}$ Hole size:4 3/4 in.Air $\square$ Water $\square$ Cored:Yes $\square$ No曰

Remarks:

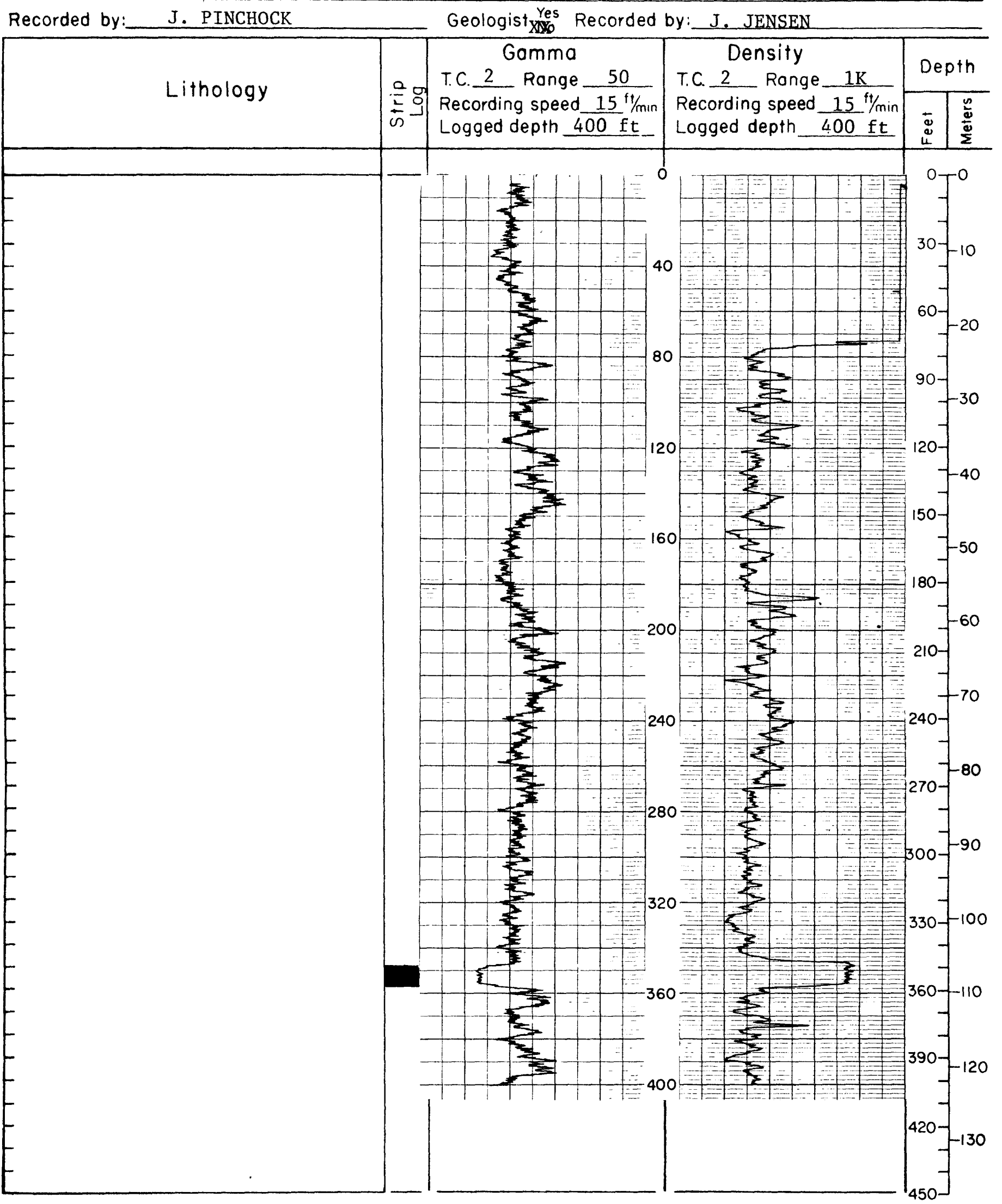




\section{Montana College of Mineral Science and Technology}

Hole no:US-77152 Mop: Fallon NE

Elev.: $2885 \mathrm{ft}$ Locotion:T. 14 N. R. 52 W. Sec. 2 , Tract CAAB _ Drilled depth: $522 \mathrm{ft}$

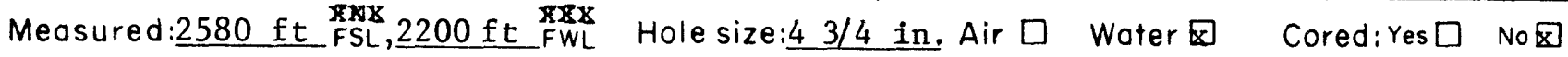
Remorks:

Recorded by:

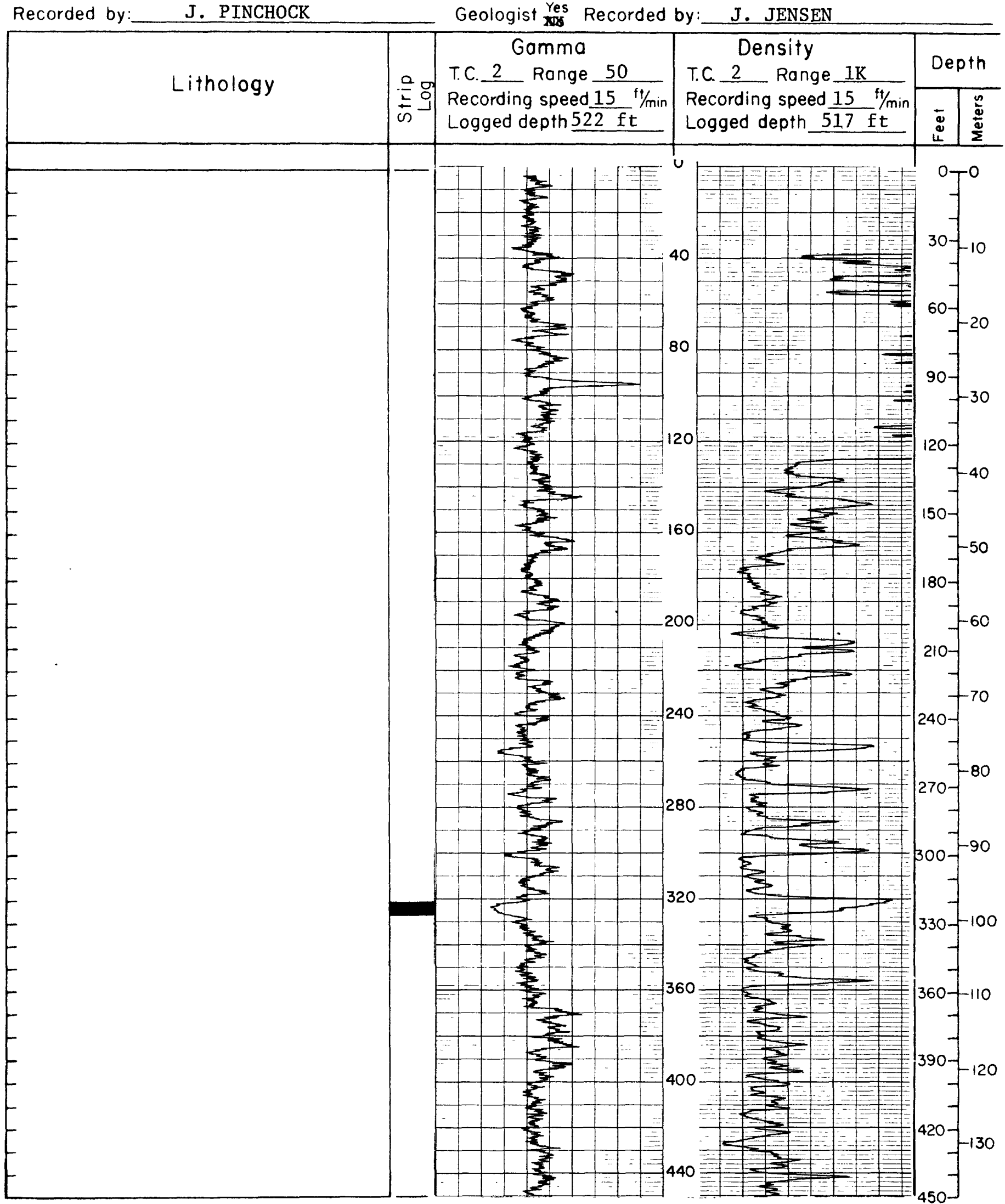


Hole number (continued)

$\underline{U S-77152}$

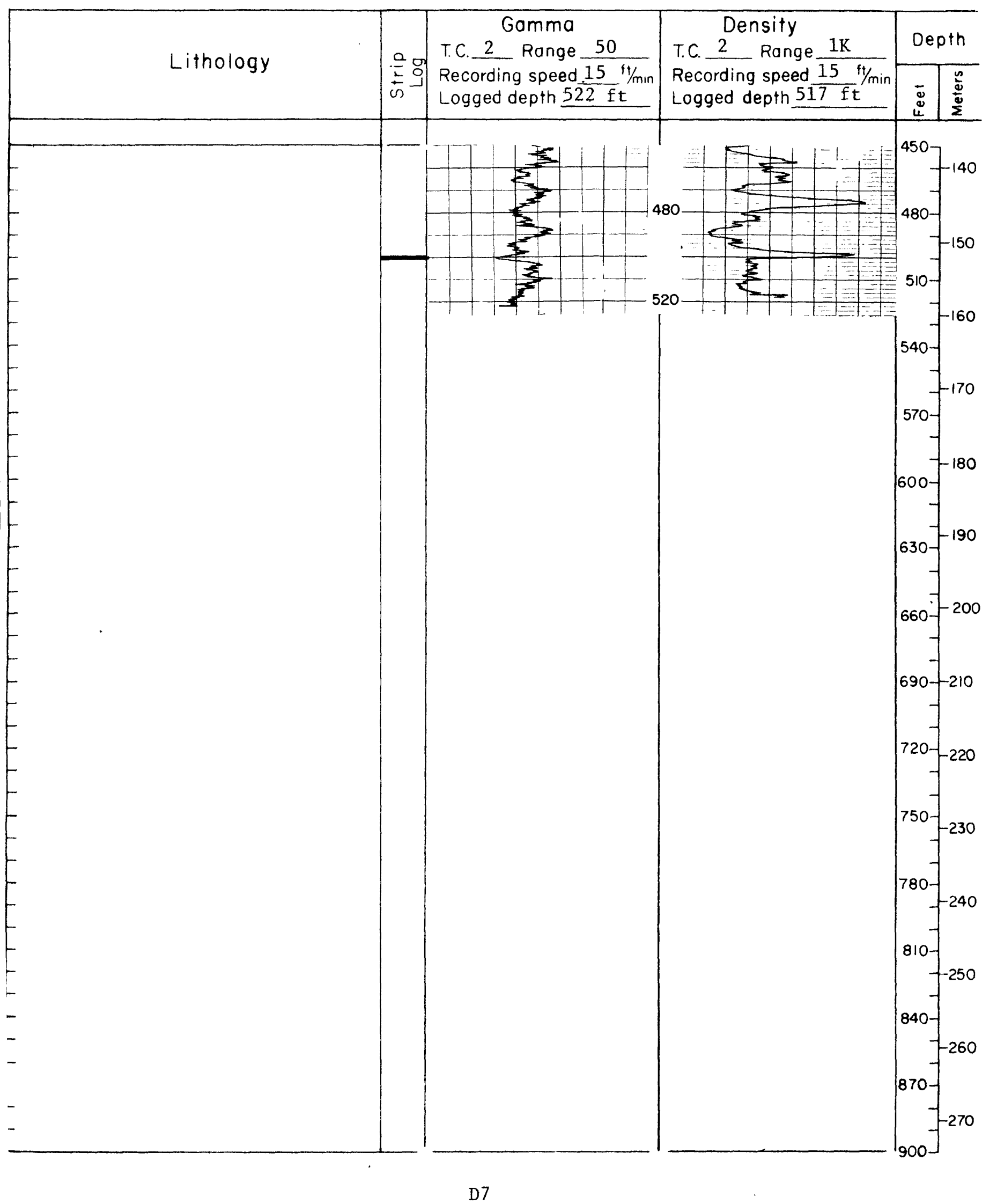


Hole number (continued)

$\underline{\text { US }-77152}$

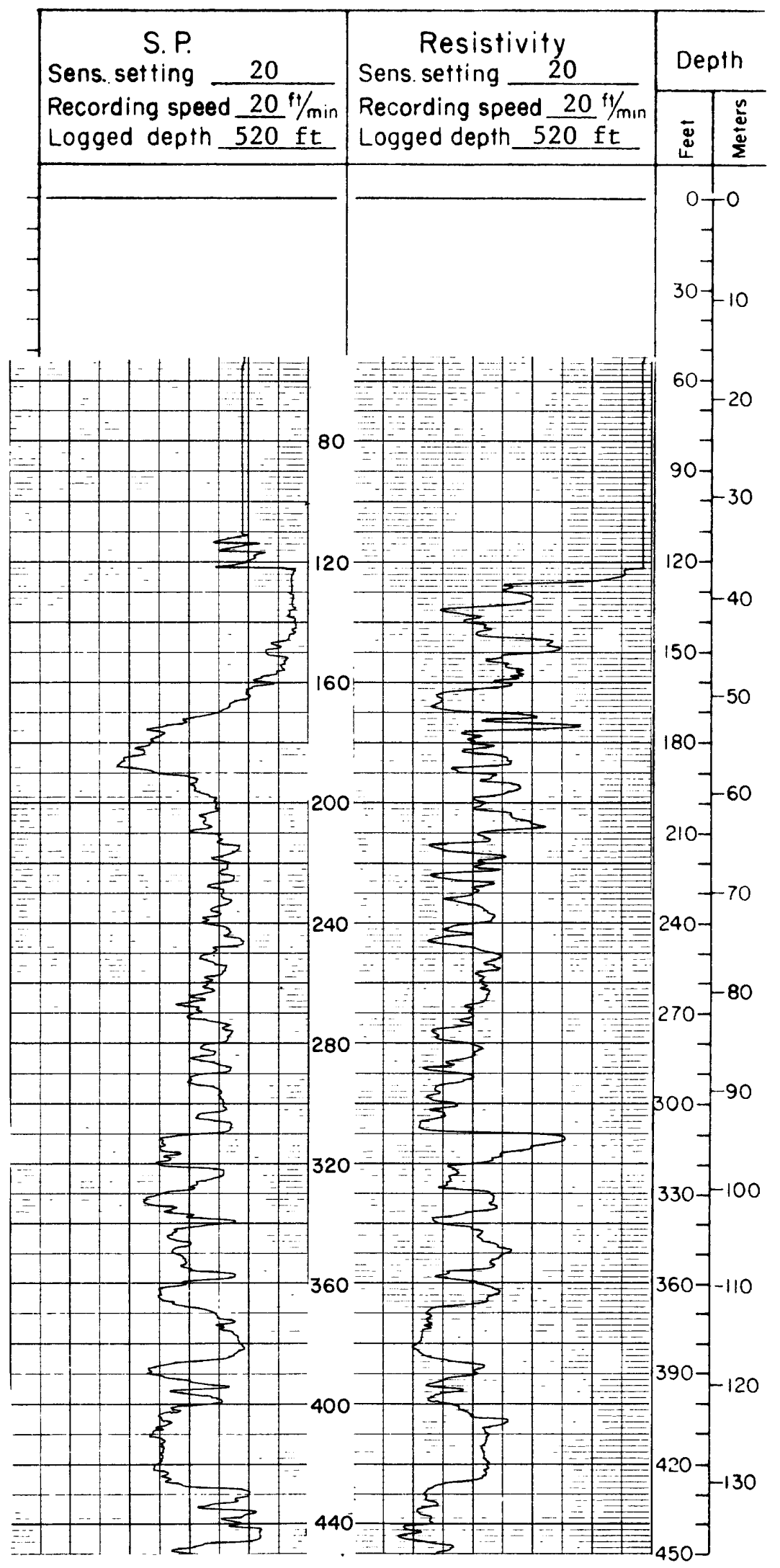


Hole number (continued)

$\underline{U S-77152}$

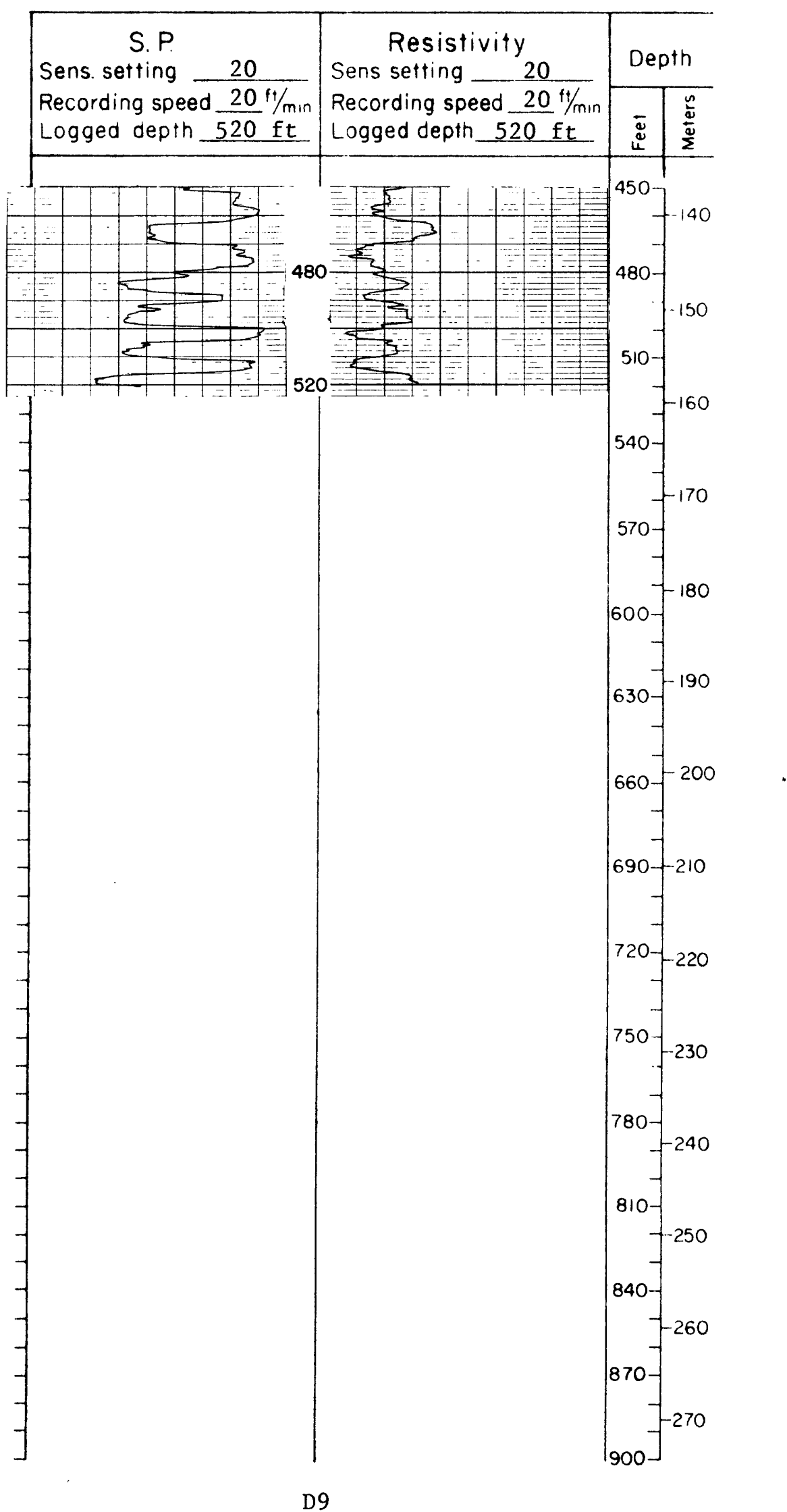


MONTANA BUREAU OF MINES AND GEOLOGY

Montana College of Mineral Science and Technology

Hole no: US-77153 Map: BLivi-ĩE 39 Date8/14/775tate:Montana County: Dawson. Elev. $2685 \mathrm{ft}$ Location: T. 15 N. R. $52 \underset{\mathrm{E}}{\mathrm{E}}$ Sec. 2 , Tract BBBB Drilled depth: $240 \mathrm{ft}$

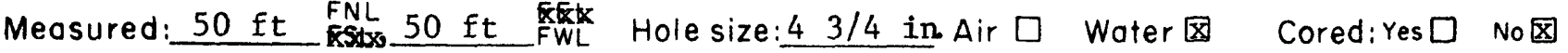
Remarks :

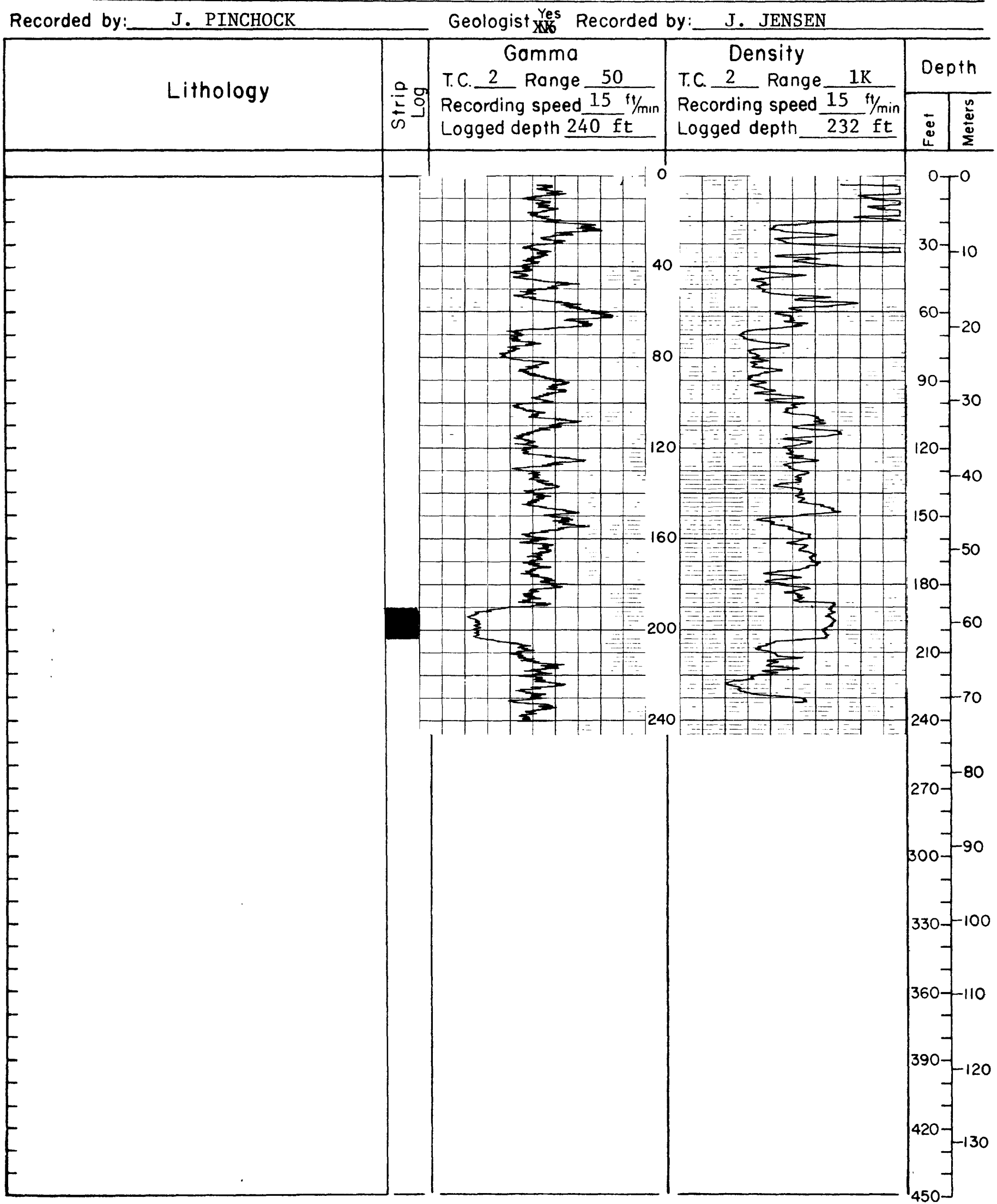


Hole number (continued)

$\underline{U S-77153}$

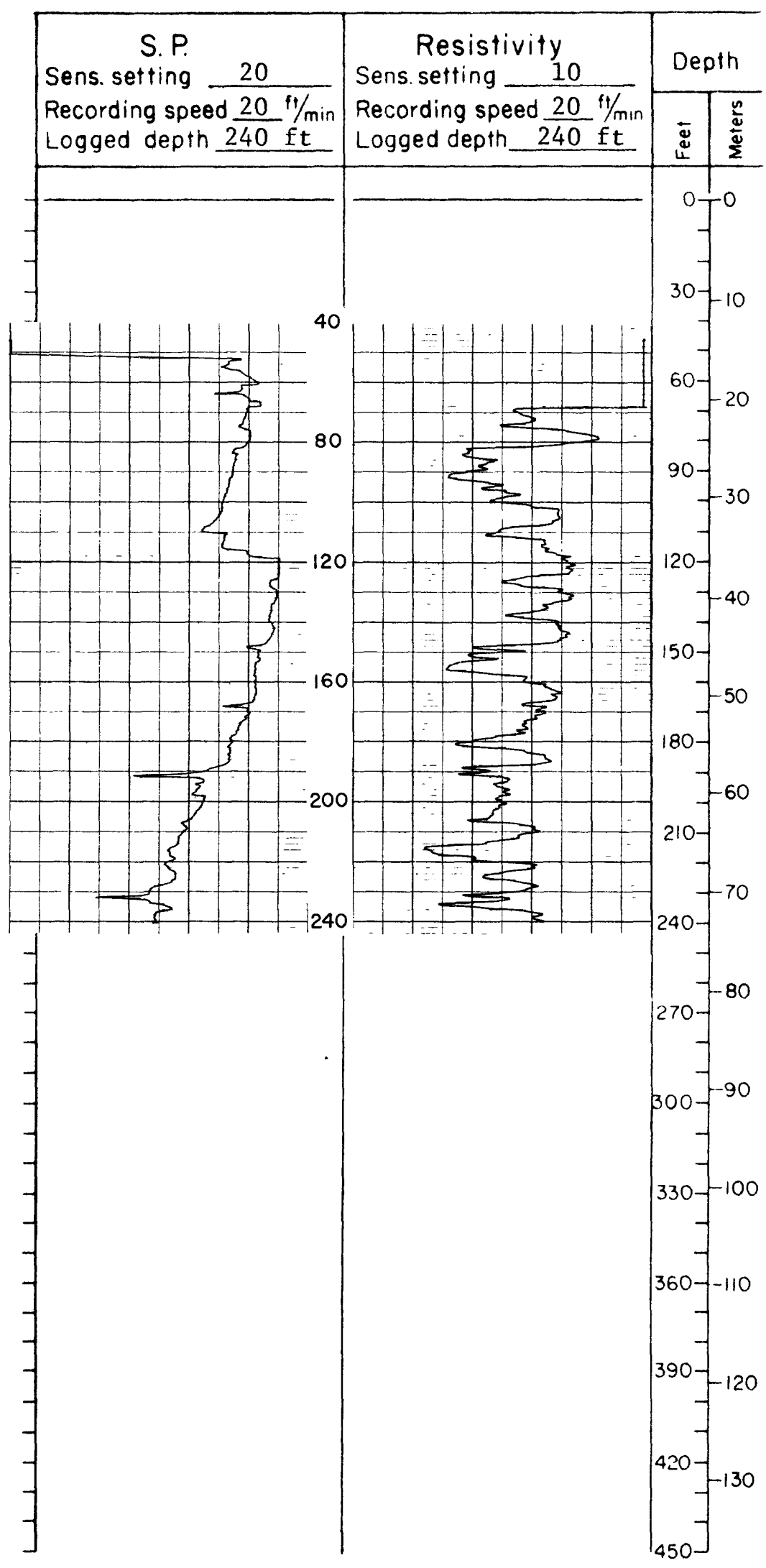

D11 
Hole no: US-77154 Map:BLM-NE 39

Elev. $2726 \mathrm{ft}$ Location: T. $15 \mathrm{~N}$ \$XR. 52 E. Sec. 4 , Tract CBBB Drilled depth: $600 \mathrm{ft}$ Meosured: $2641 \mathrm{ft}$ FNL, $50 \mathrm{ft}$ Holesize4 3/4 in. Air $\square$ Water $\square$ Cored:Yes $\square$ No $\square$ Remarks:

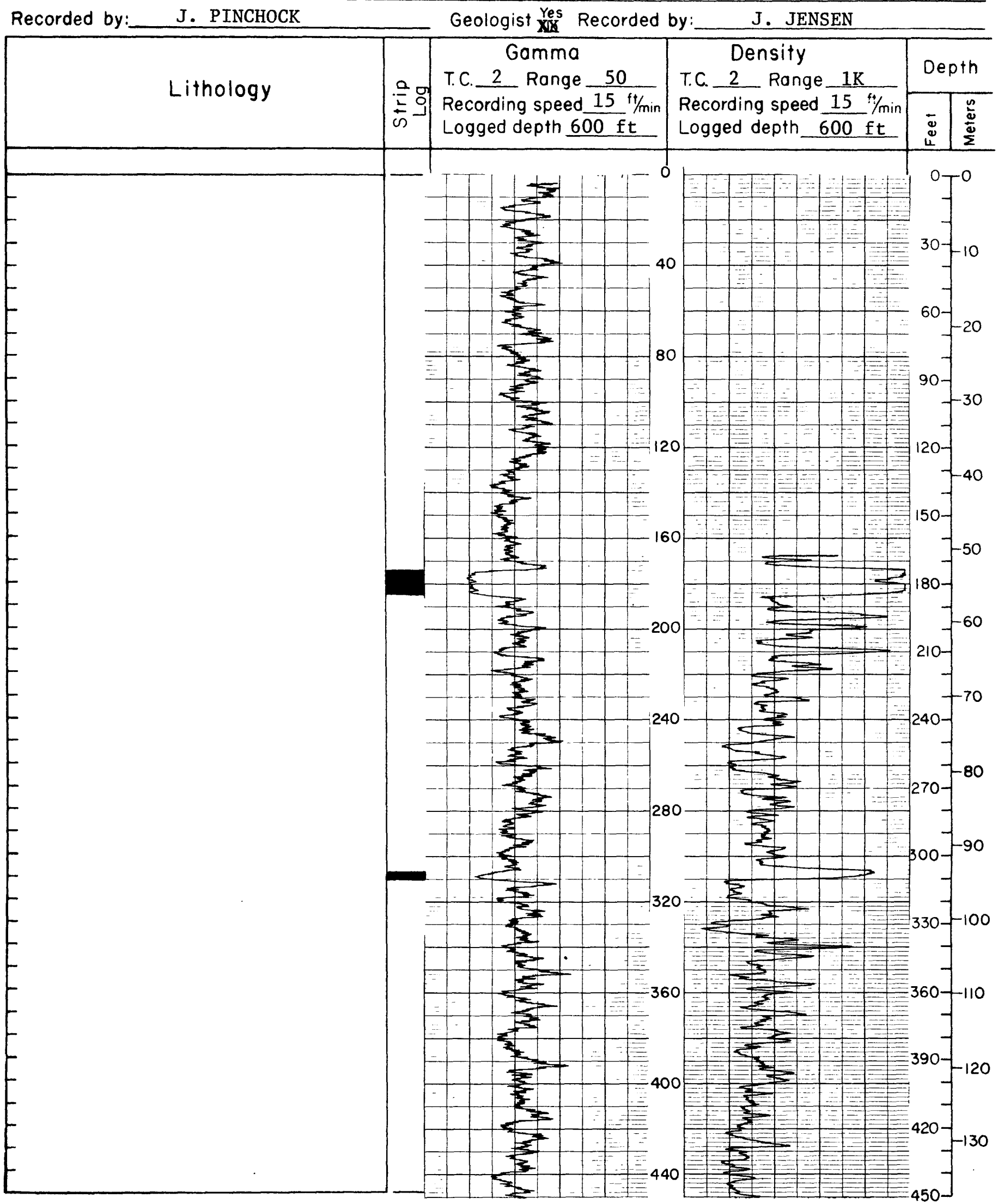


Hole number (continued)

US - 77154

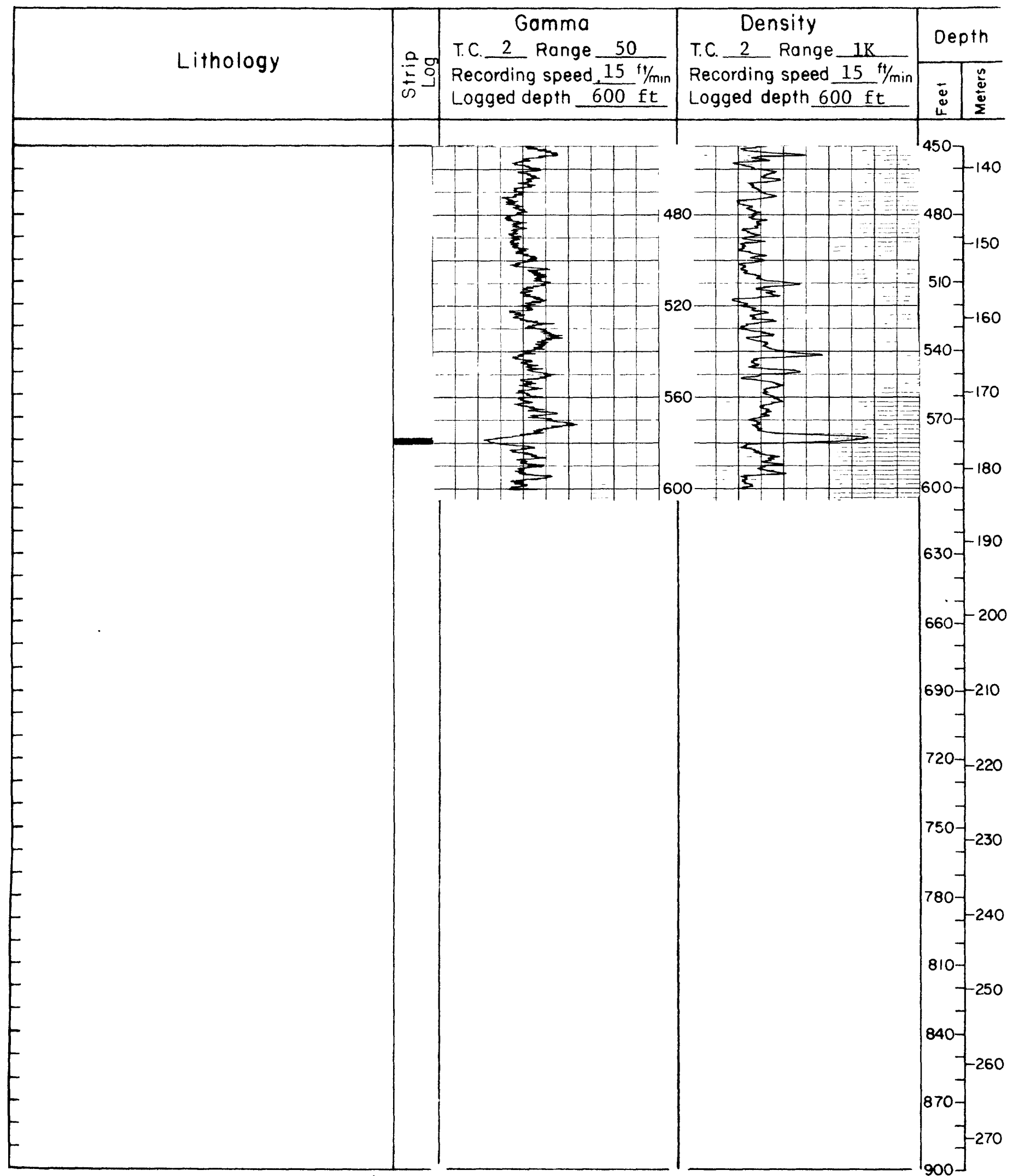


Hole number (continued)

US-77154

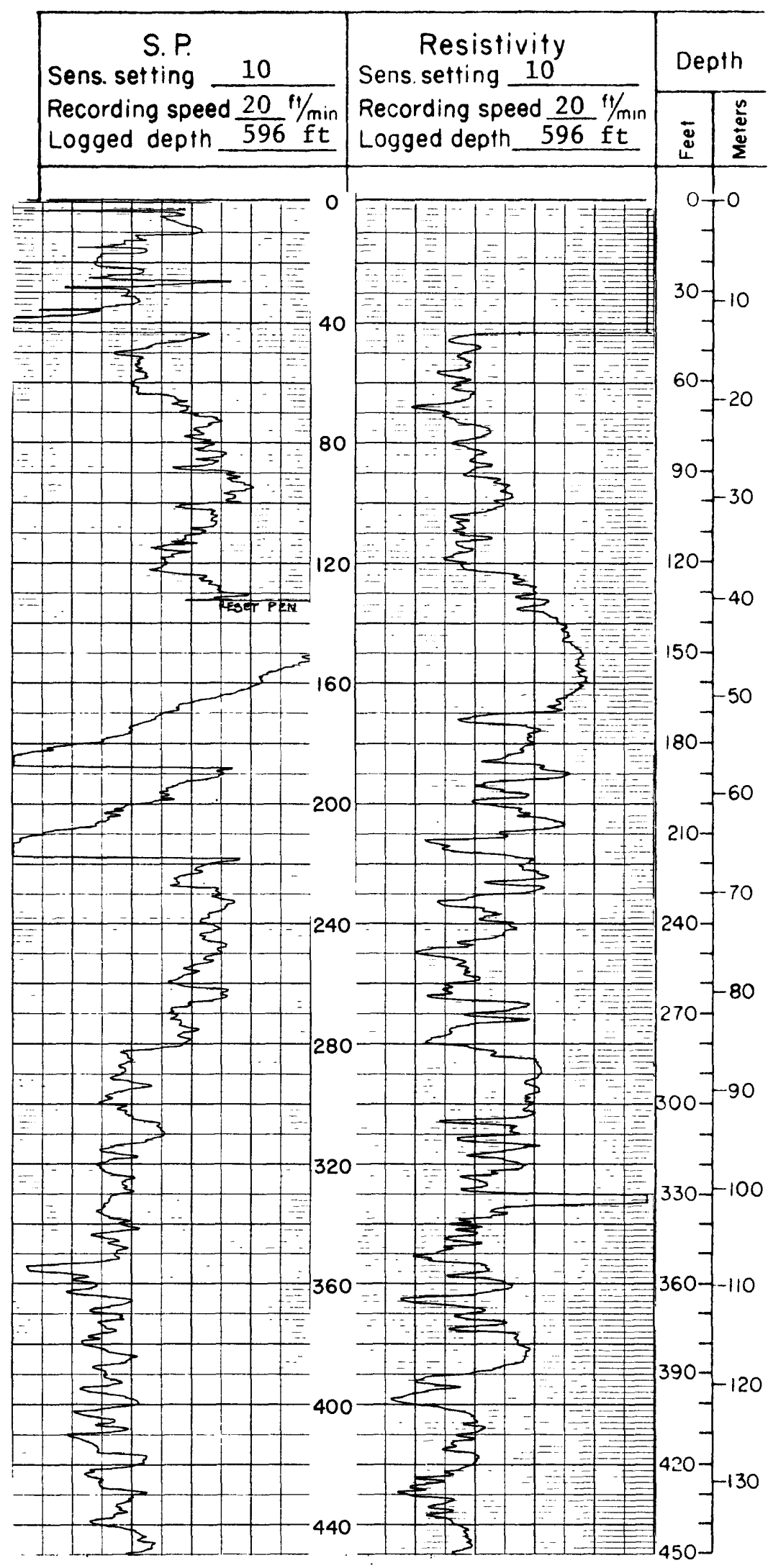


Hole number (continued)

US -77154

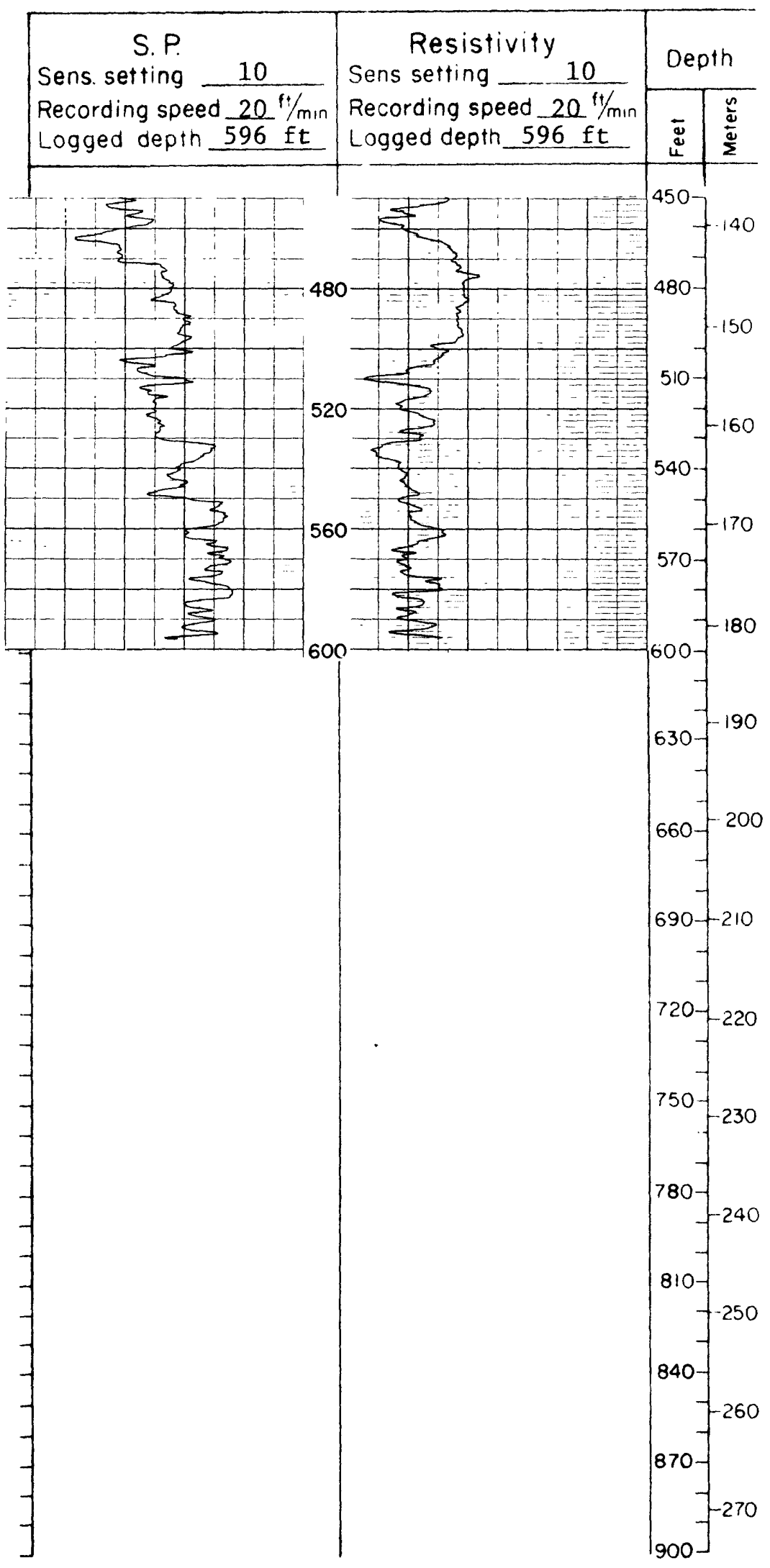

$\mathrm{D}_{15}$ 
MONTANA BUREAU OF MINES AND GEOLOGY

Montana College of Mineral Science and Technology

Hole no: US-77155 Map: BLM-NE 39

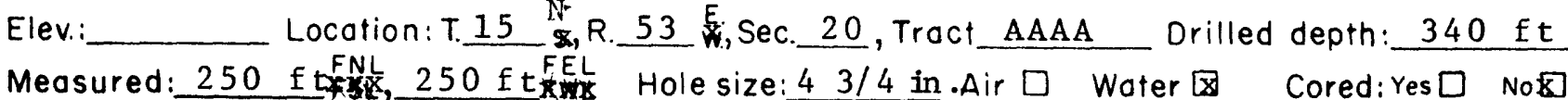

Date:9/20/77State: MontanaCounty: Dawson

Remarks:

Recorded by

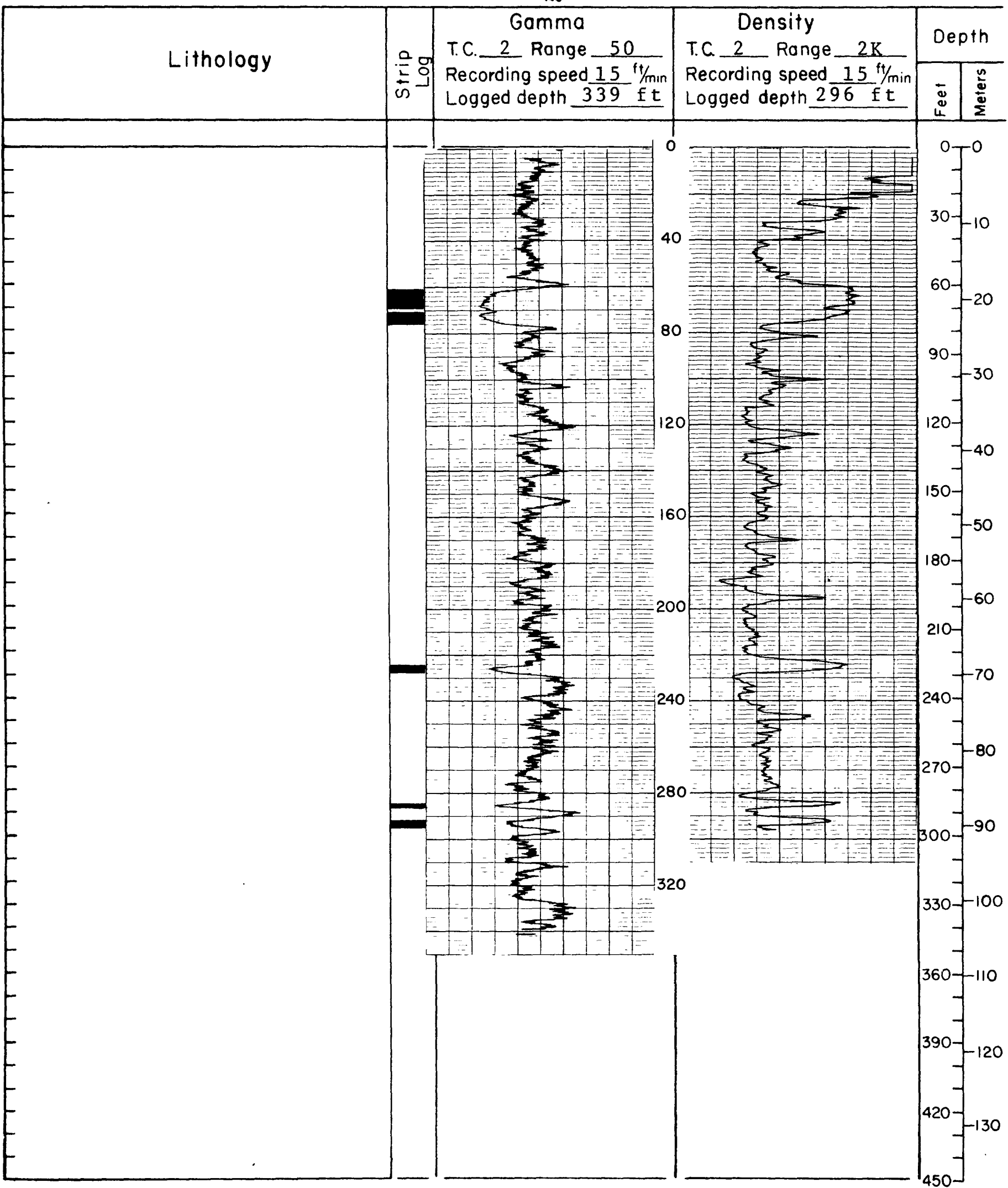


Hole number (continued)

$\underline{U S-77155}$

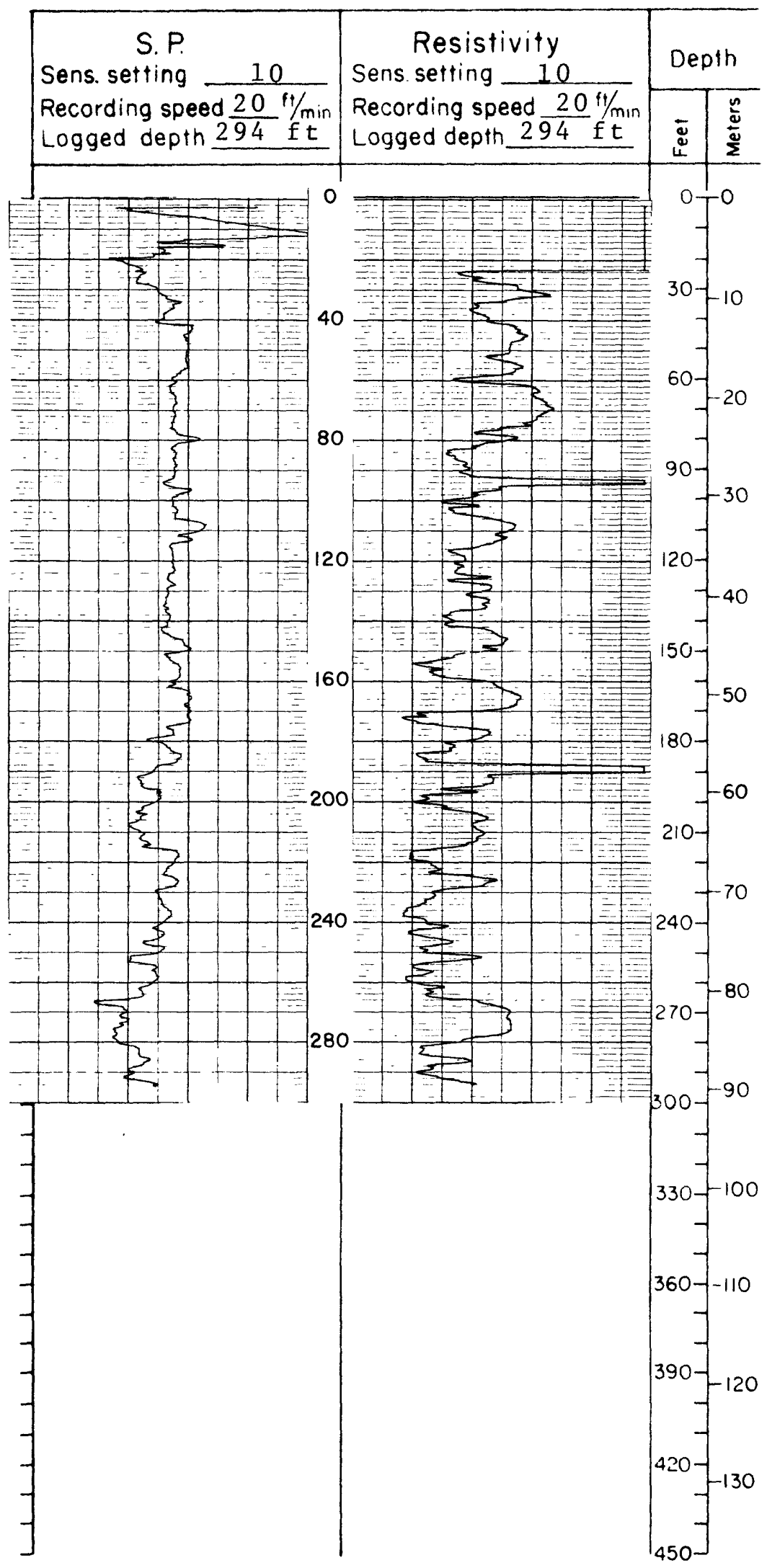


Hole no: US-77156 MOP:BLM-NE 39

Date $9 / 20 / 77$ State: Montana County: Dawson Elev. $2855 \mathrm{ft}$ Location: T. 15 N. R. 53 E. Sec. 4 , Tract CCCD Drilled depth: $400 \mathrm{ft}$ Measured: $60 \mathrm{ft}$ FSL, $400 \mathrm{ftFWL}$ Hole size:43/4 in. Air $\square$ Woter $\mathrm{F}$ Cored:Yes $\square$ No

Remorks:

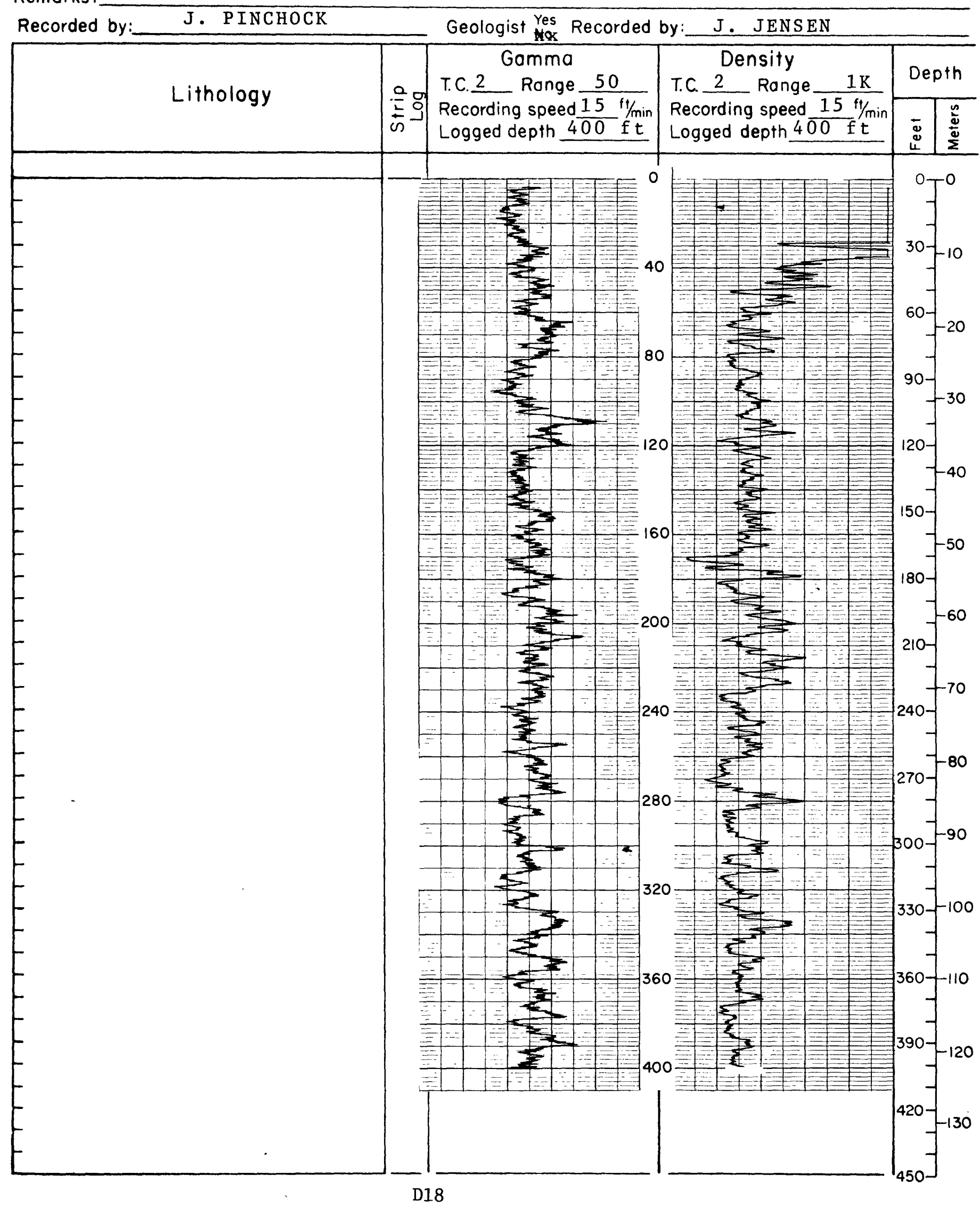


Hole number (continued)

$\underline{U S-77156}$

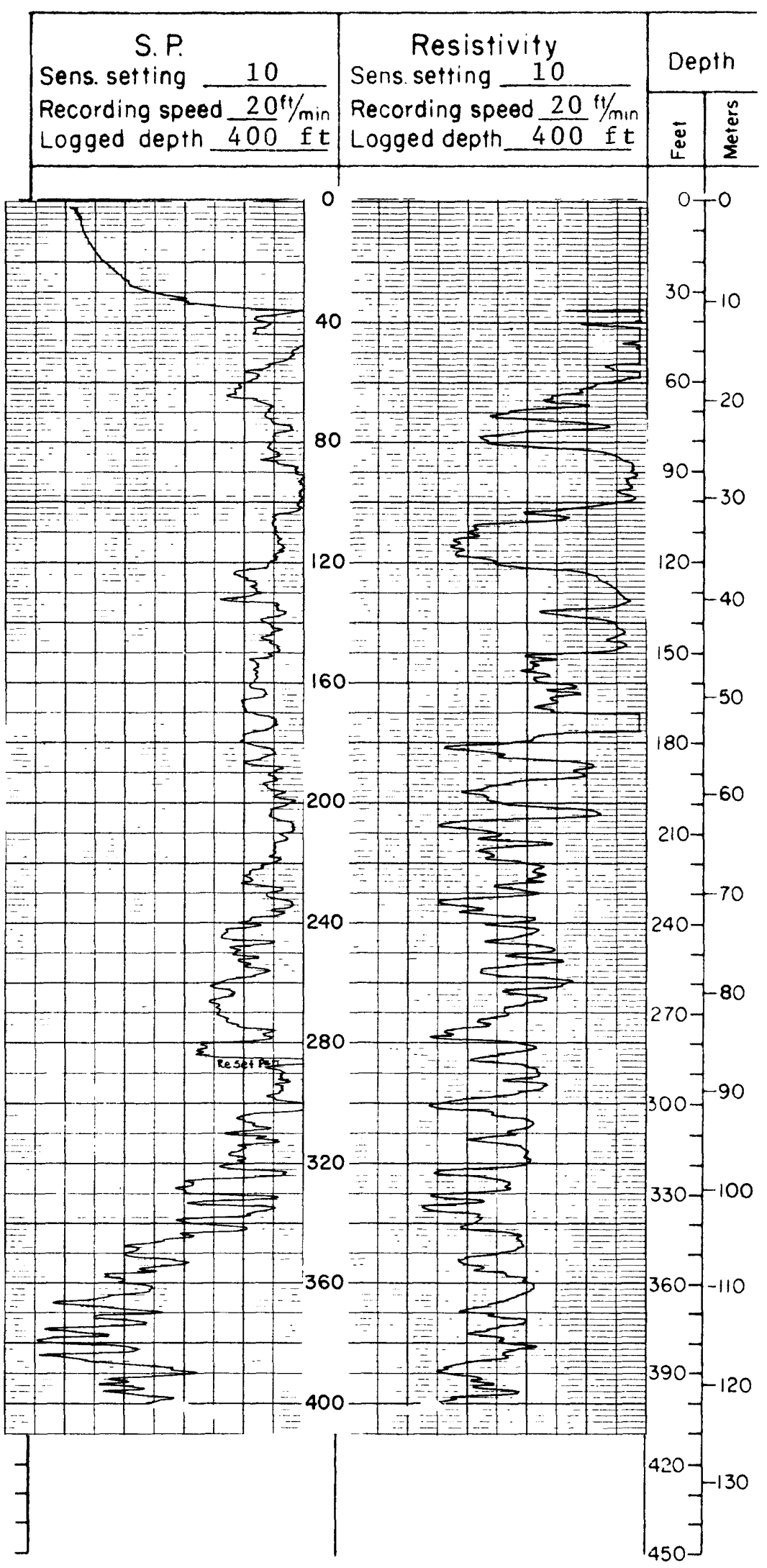




\section{MONTANA BUREAU OF MINES AND GEOLOGY \\ Montana College of Mineral Science and Technology}

Hole no:US-77157 Map:Pleasant View Date9/22/77 State:Montana County:Dawson Elev. $2570 \mathrm{ft}$ Locotion: T. $16 \mathrm{~N}, \mathrm{~N}$. $54 \mathrm{E}, \mathrm{Eec} .32$, Tract_ CCCC Drilled depth: $400 \mathrm{ft}$

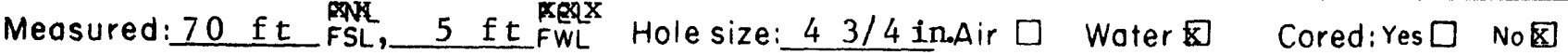
Remarks:

Recorded by: J. PINCHOCK Geologist $\underset{\text { No }}{\text { Yes }}$ Recorded by: J. JENSEN

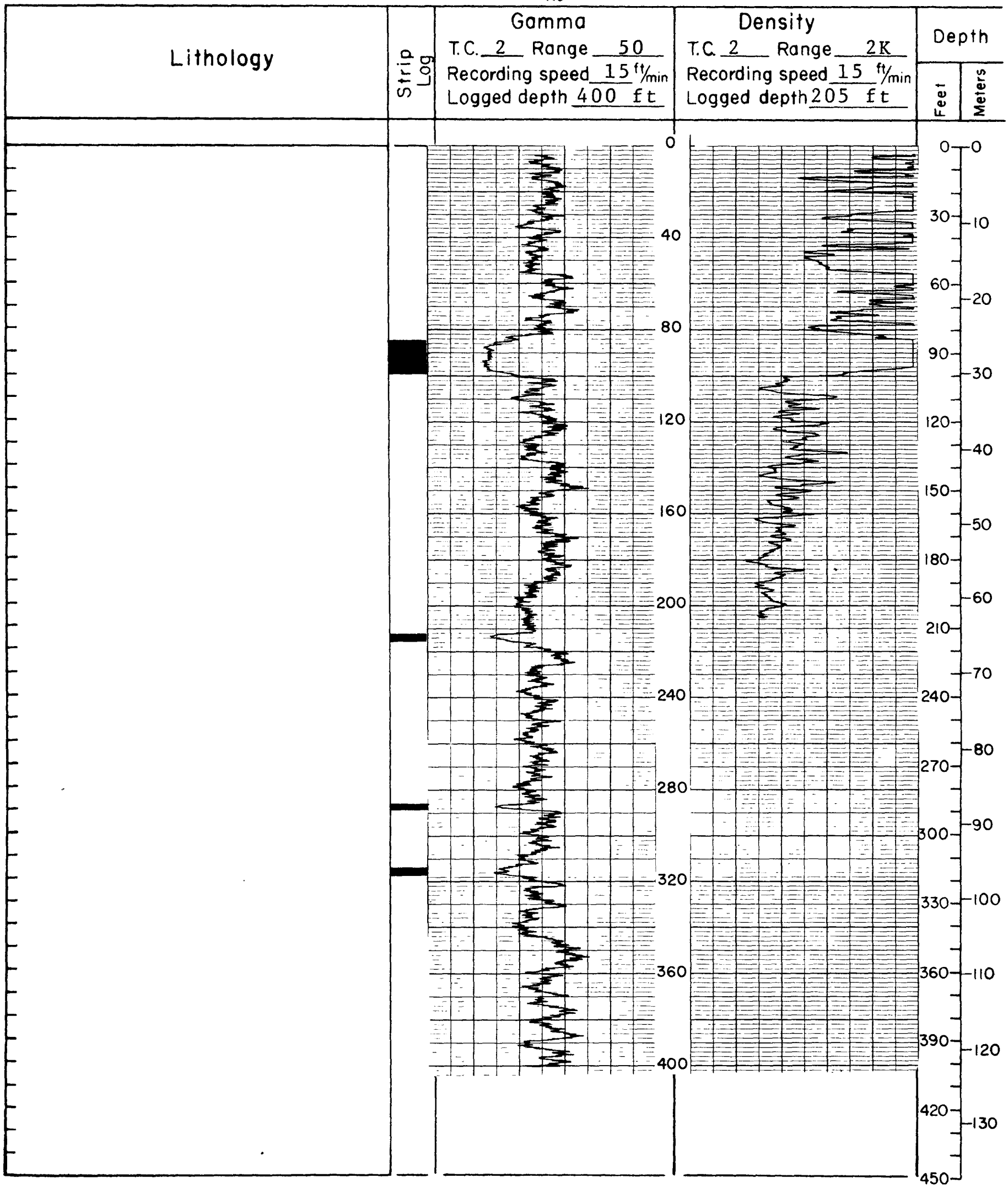


Hole number (continued)

US-77157

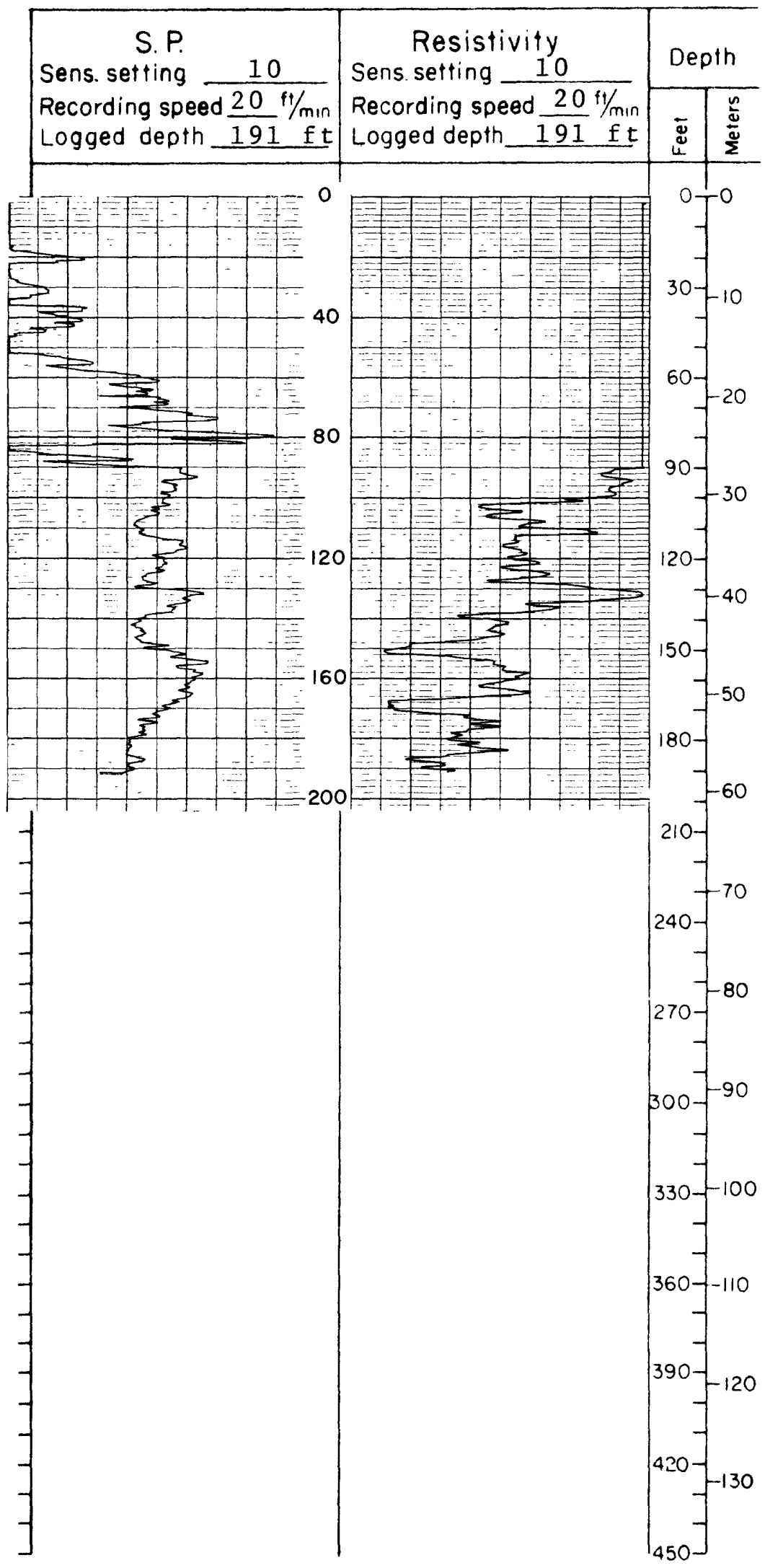


Hole no:US-77158 Map:Poverty Flat WestDote $9 / 21 / 77$ State: Montana County: Dawson Elev. $2446 \mathrm{ft}$ Location: T. $16 \mathrm{~N}$. R. $53 \mathrm{E}$, Sec. 12 , Tract CCBC Drilled depth: $100 \mathrm{ft}$

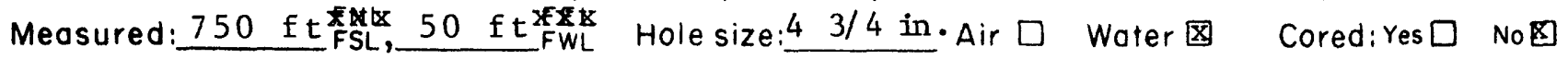

Remorks:

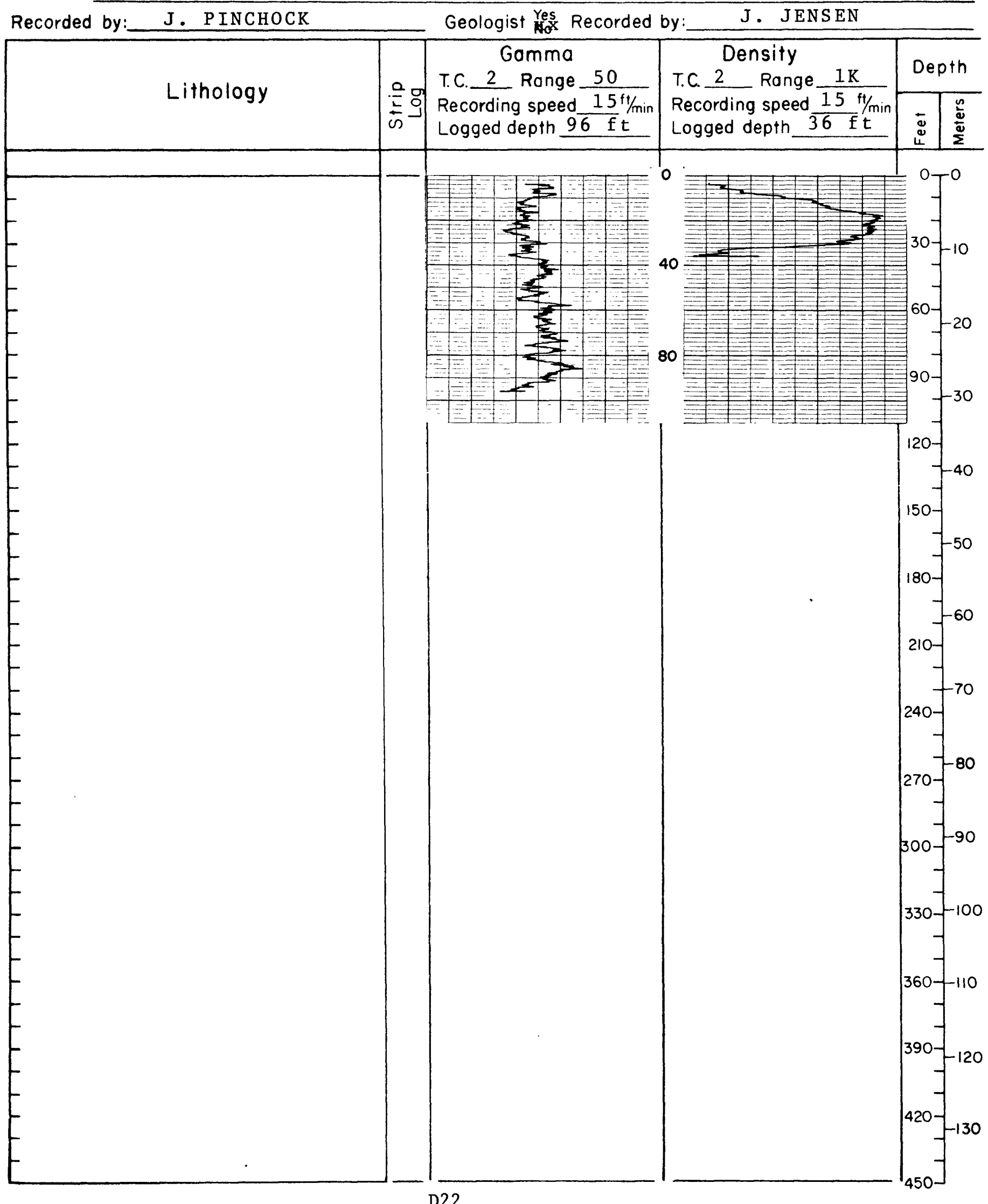


Hole number (continued)

$\underline{U S-77158}$

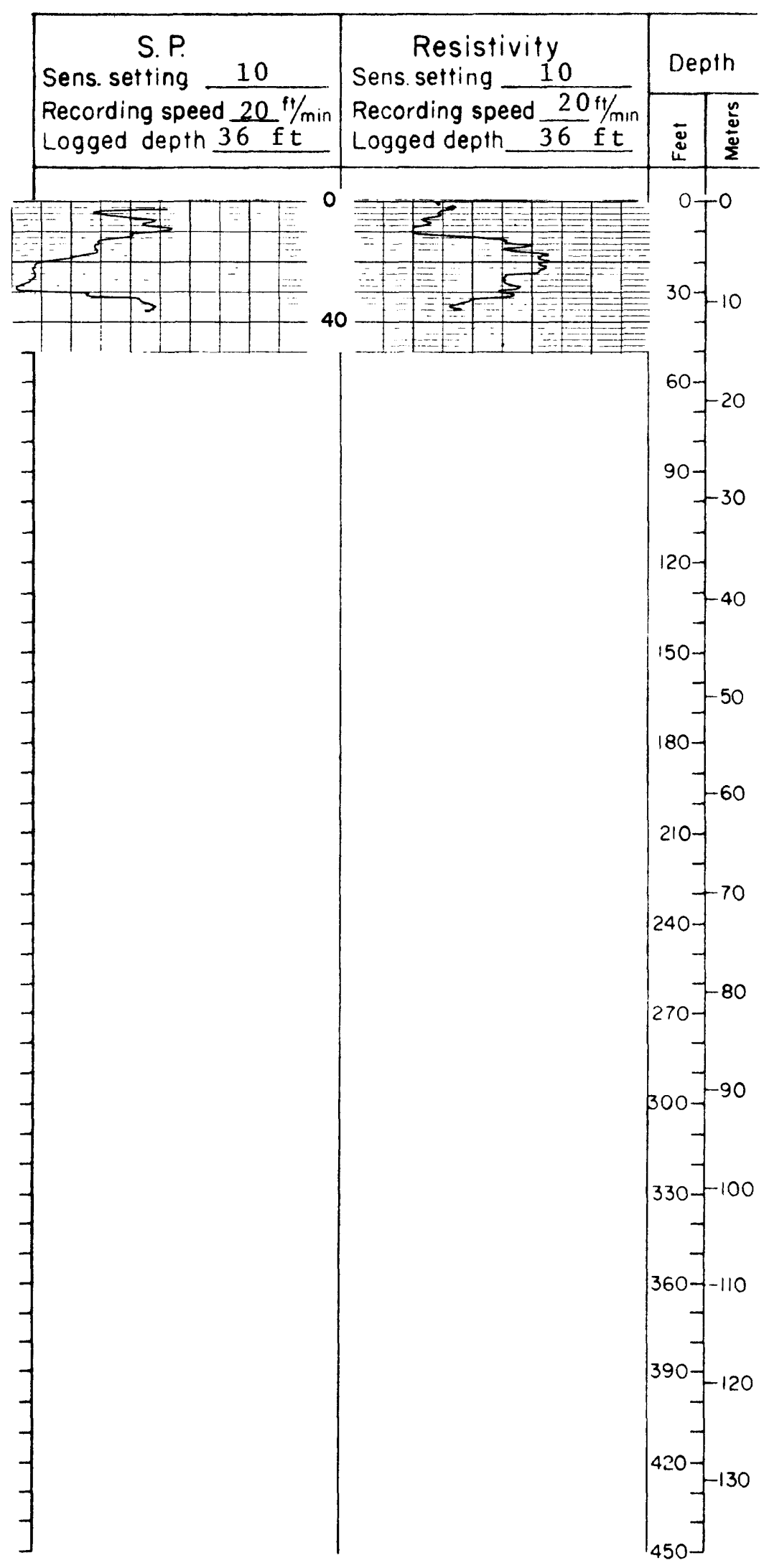




\section{MONTANA BUREAU OF MINES AND GEOLOGY \\ Montana College of Mineral Science and Technology}

Hole no:US-77159 MapPoverty Flat West Date9/21/77State:Montana County: Dawson

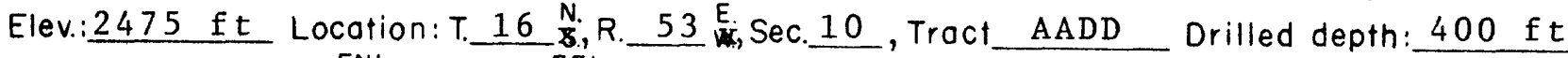

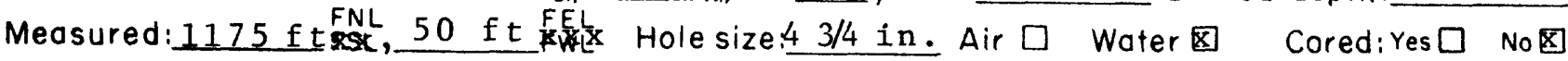
Remarks:

Recorded by:_ J. PINCHOCK

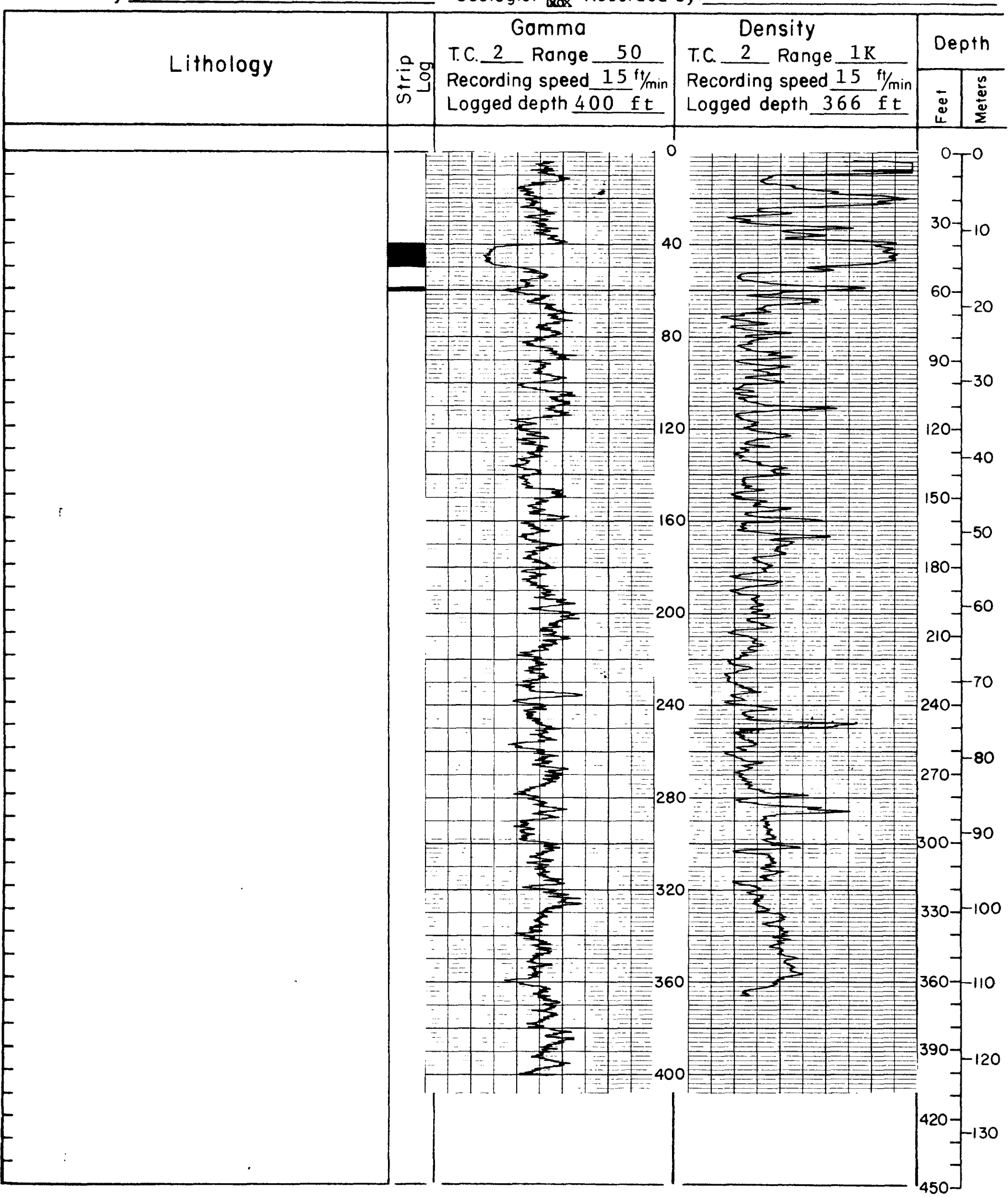


Hole number (continued)

$\underline{U S-77159}$

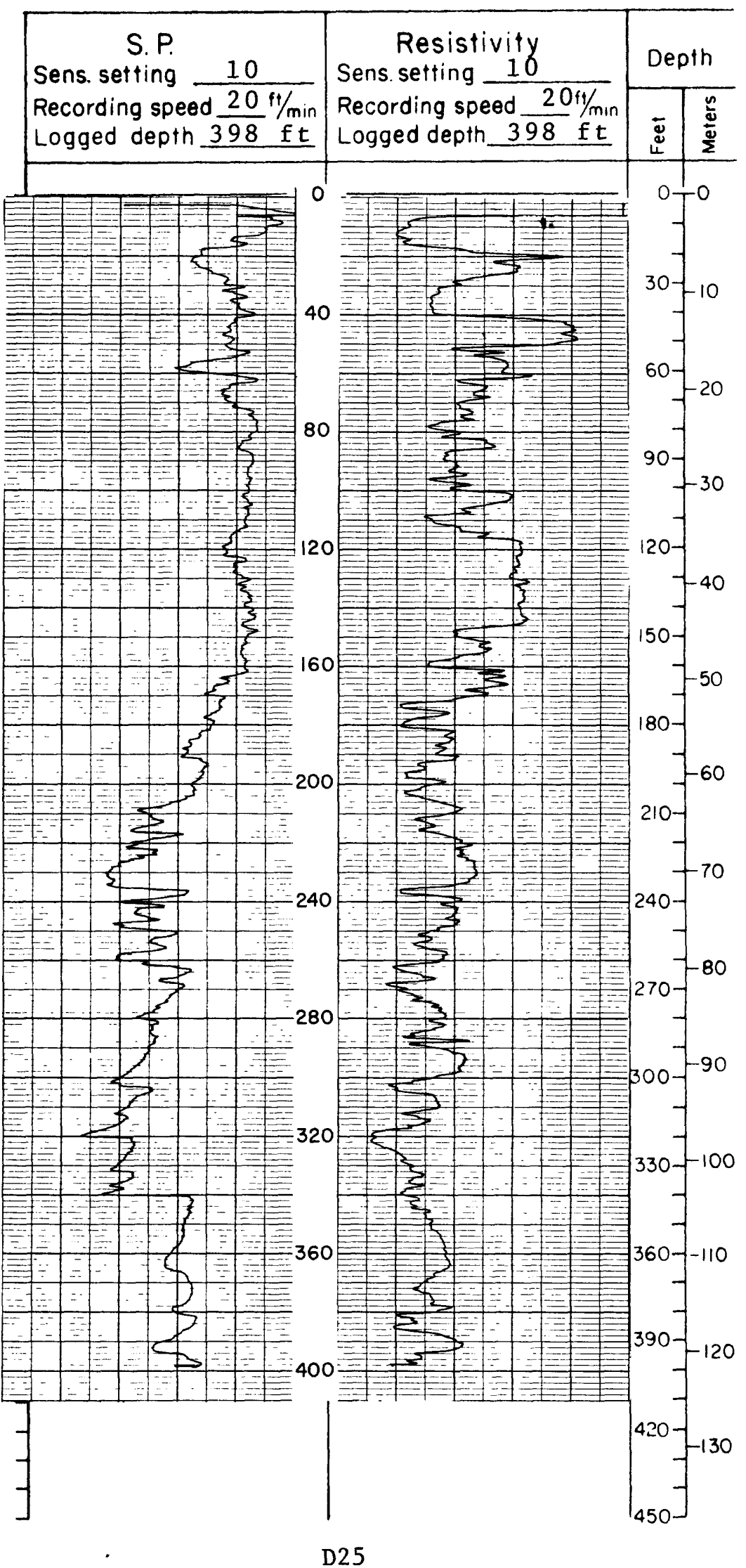




\section{MONTANA BUREAU OF MINES AND GEOLOGY \\ Montana College of Mineral Science and Technology}

Hole no:US-77160 MapPoverty Flat West Date9/25/77 State: MontanaCounty: Dawson Elev. $2750 \mathrm{ft}$ Location: T. 16 N. R. 54 E. Sec. 6, Tract CDDB. Drilled depth: $340 \mathrm{ft}$

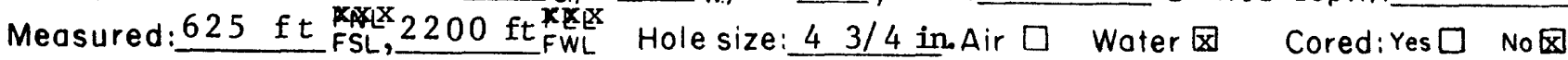

Remarks:

Recorded by: J. PINCHOCK Geologist Yes Recorded by:_J. JENSEN

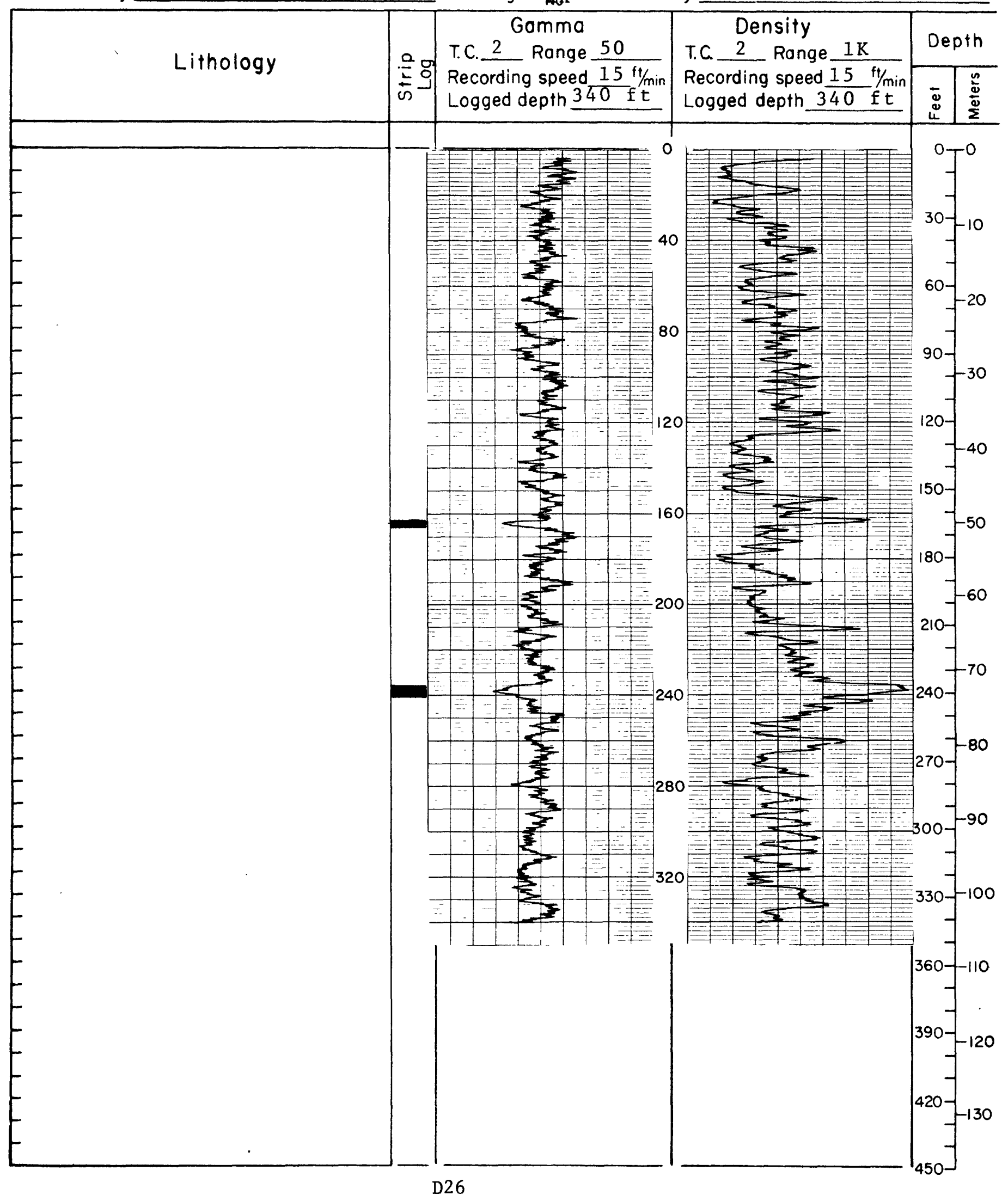


Hole number (continued)

$\underline{U S-77160}$

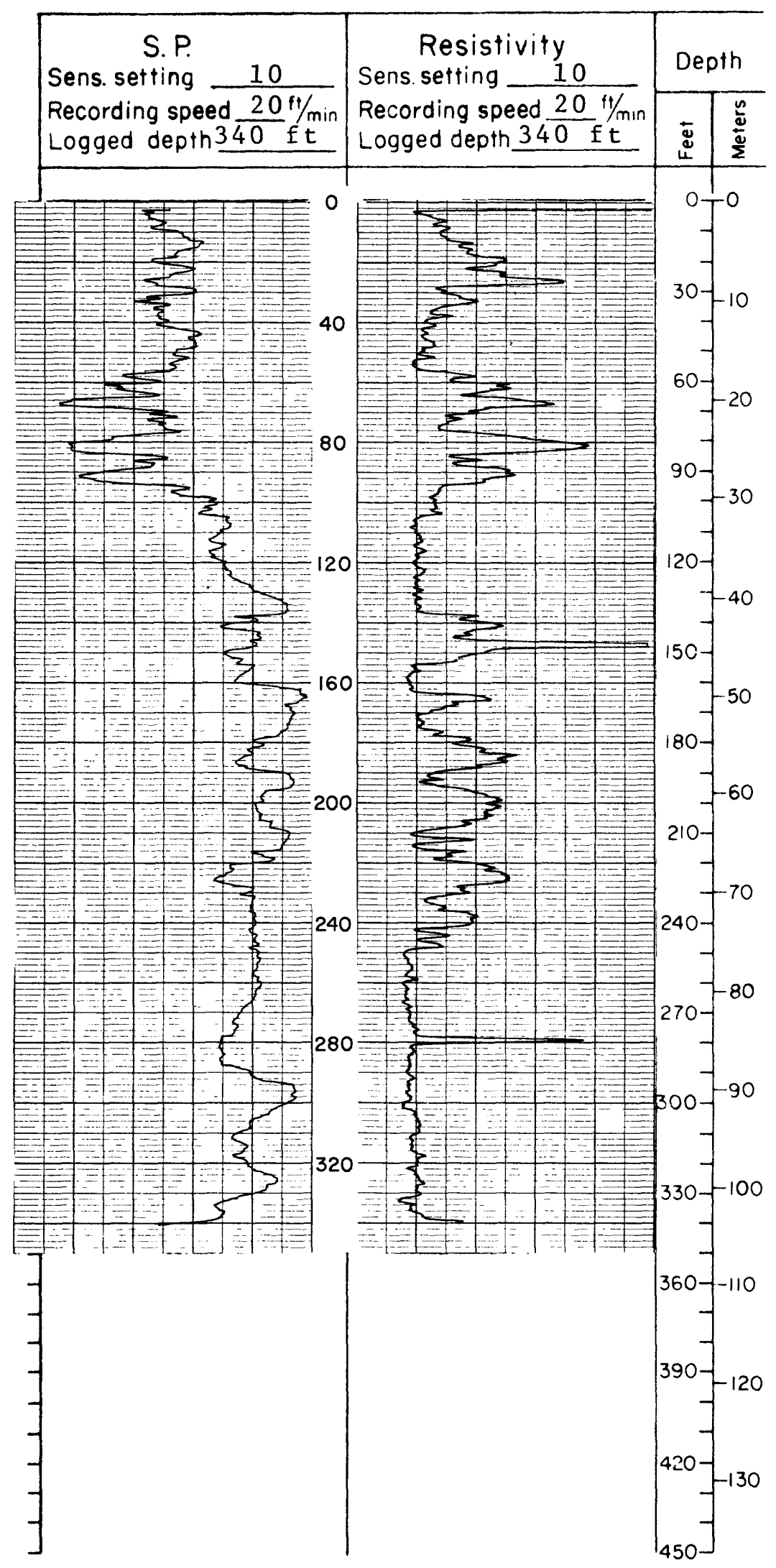




\section{MONTANA BUREAU OF MINES AND GEOLOGY \\ Montana College of Mineral Science and Technology}

Hole no: US-77161 MapKo1berg Ranch Date9/26/77 StateMontana County: Dawson Elev. $2327 \mathrm{ft}$ Location: T. $17 \stackrel{\mathrm{N}}{\mathrm{S}}, \mathrm{R} .55 \mathrm{~W} . \mathrm{Sec} .6$, Tract AAAA Drilled depth: $260 \mathrm{ft}$ Measured: $250 \mathrm{ftFNL} 20 \mathrm{ft} F$

Remarks :

Recorded by: J. PINCHOCK Geologist Yes Recorded by: J. JENSEN

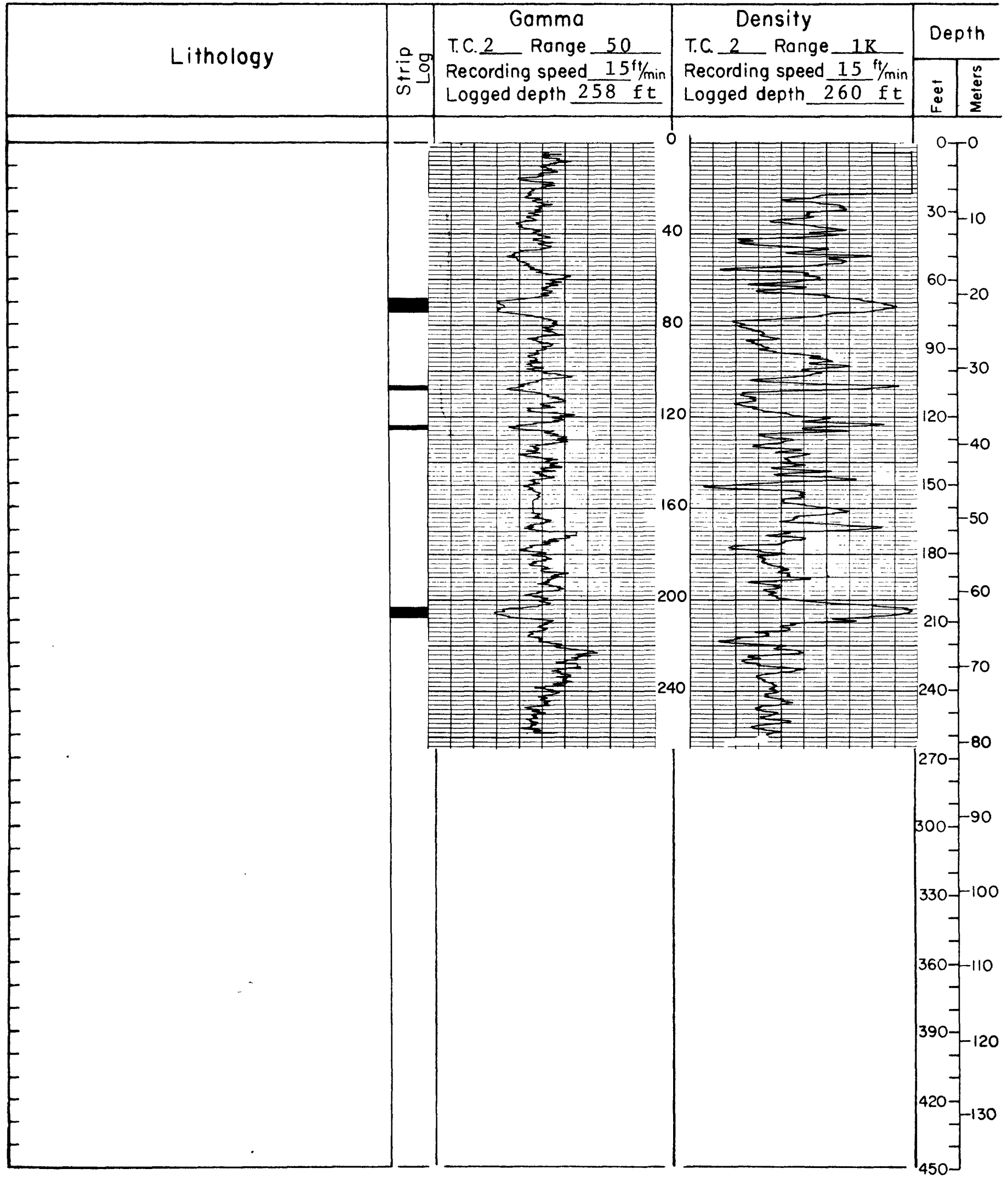


Hole number (continued)

$\underline{U S-77161}$

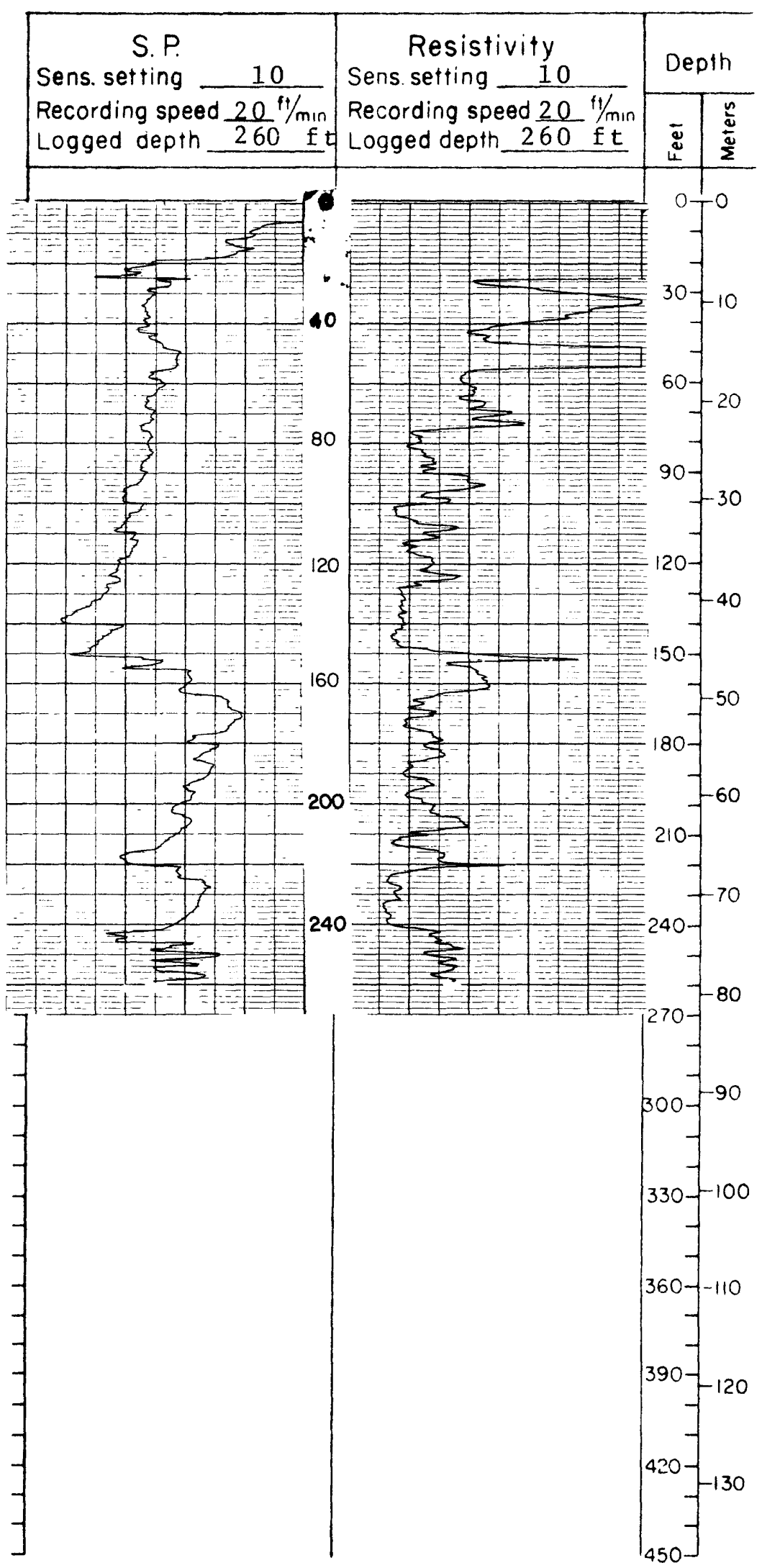




\section{MONTANA BUREAU OF MINES AND GEOLOGY \\ Montana College of Mineral Science and Technology}

Hole no: US-77162 MapPoverty F1at EastDate:9/26/77StateMontana County: Dawson Elev. $2543 \mathrm{ft}$ Location: T. 16 N. R. 54 E. Sec. 10 , Tract DAAA Drilled depth: $260 \mathrm{ft}$

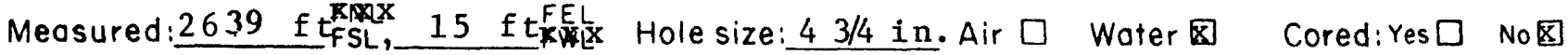

Remarks:

Recorded by:_ J. PINCHOCK

Lithology

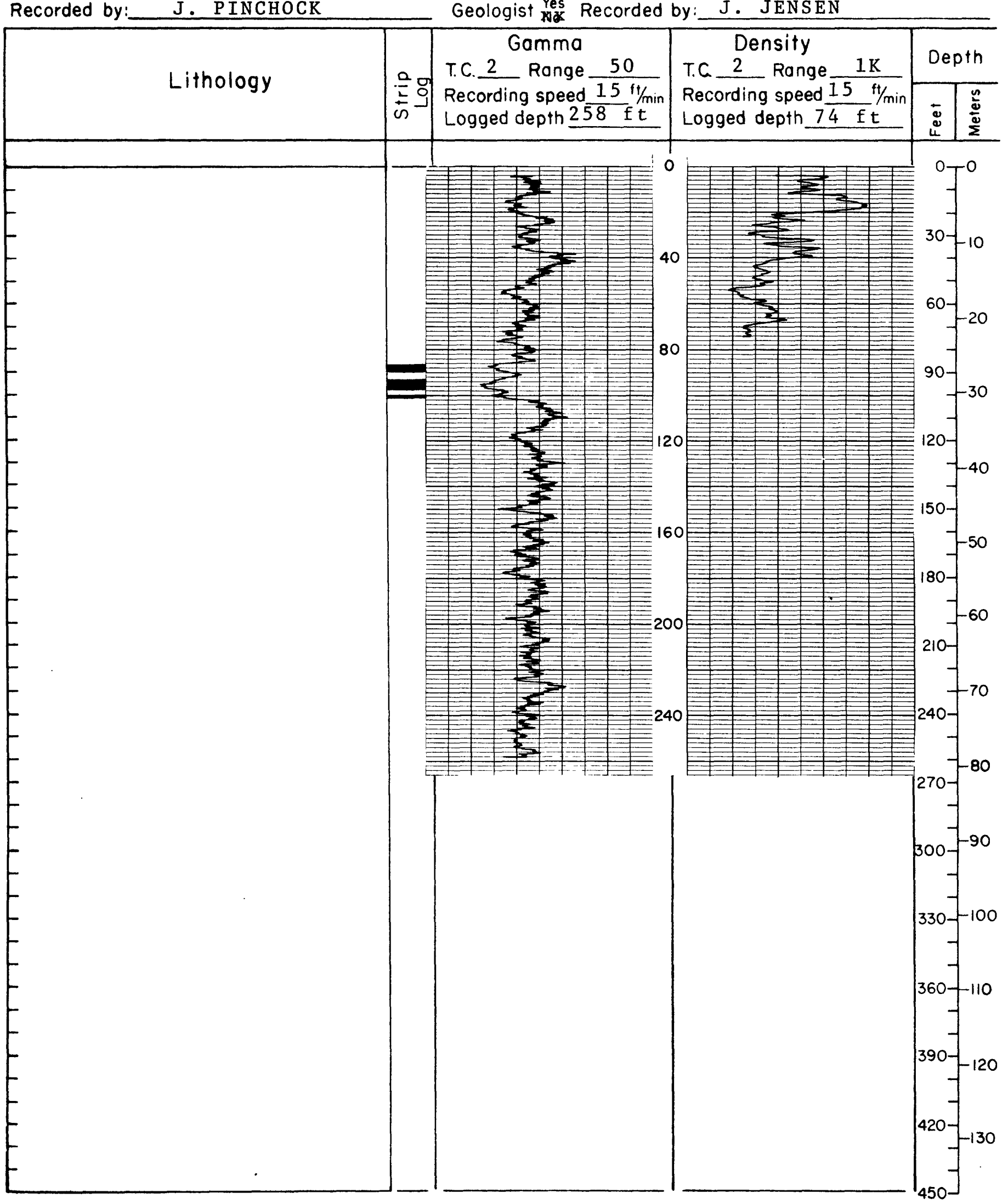




\section{MONTANA BUREAU OF MINES AND GEOLOGY \\ Montana College of Mineral Science and Technology}

Hole no:US-77163 MopPoverty Flat WestDote $9 / 26 / 77$ State:Montana County: Dawson Elev.: $2580 \mathrm{ft}$ Location: T. $16 \stackrel{\mathrm{N}}{\mathrm{z}}, \mathrm{R} . \mathbf{5 4} \mathrm{E}, \mathrm{Sec} .4$, Tract DDCD Drilled depth: $180 \mathrm{ft}$ Measured: $300 \mathrm{ft} F S L, 920 \mathrm{ftFEL}$ Hole size: $43 / 4 \mathrm{in}$. Air $\square$ Water $\mathbf{F}$ Cored:Yes $\square$ No $\otimes$

Remarks :

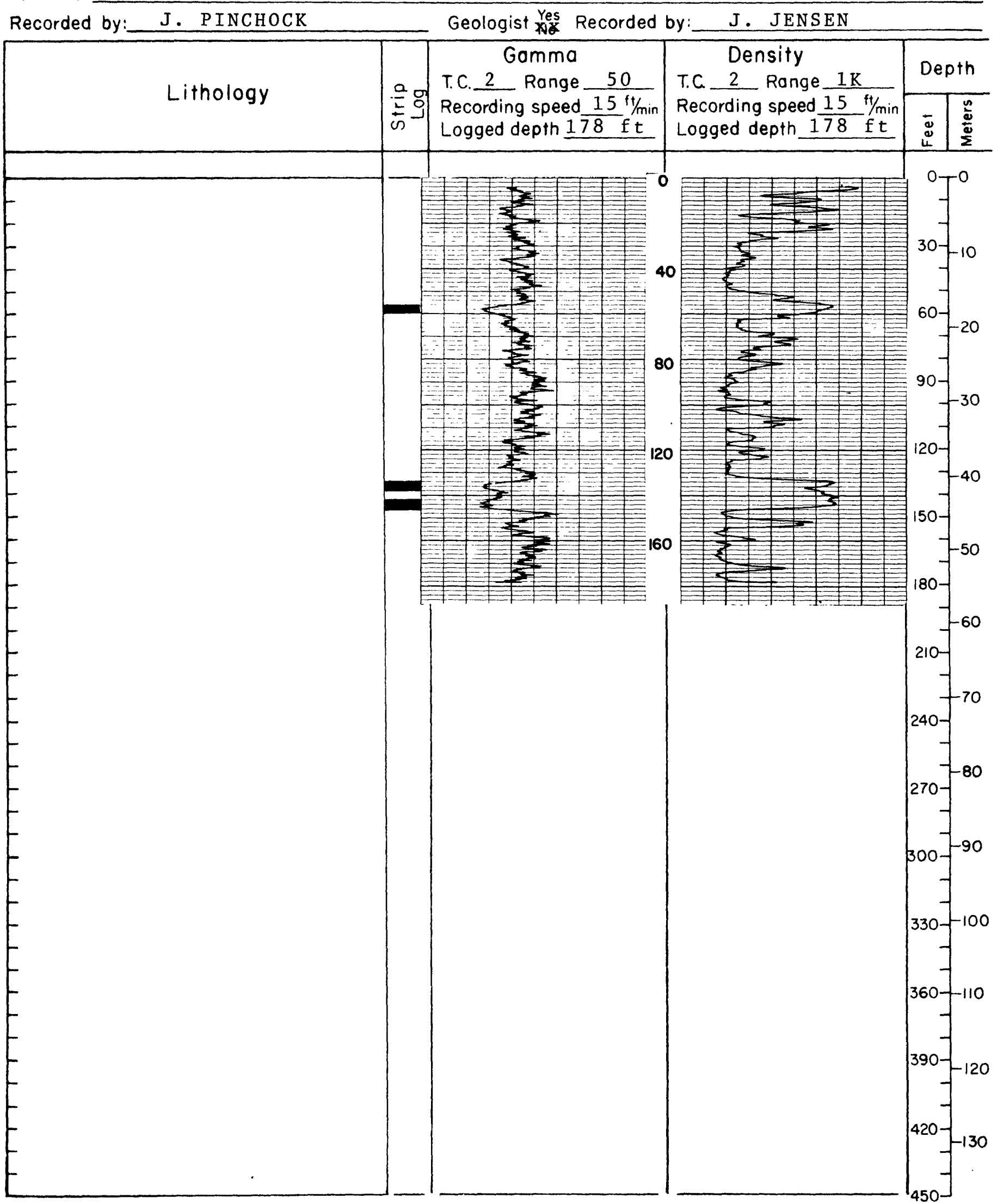


Hole number (continued)

$\underline{U S-77163}$

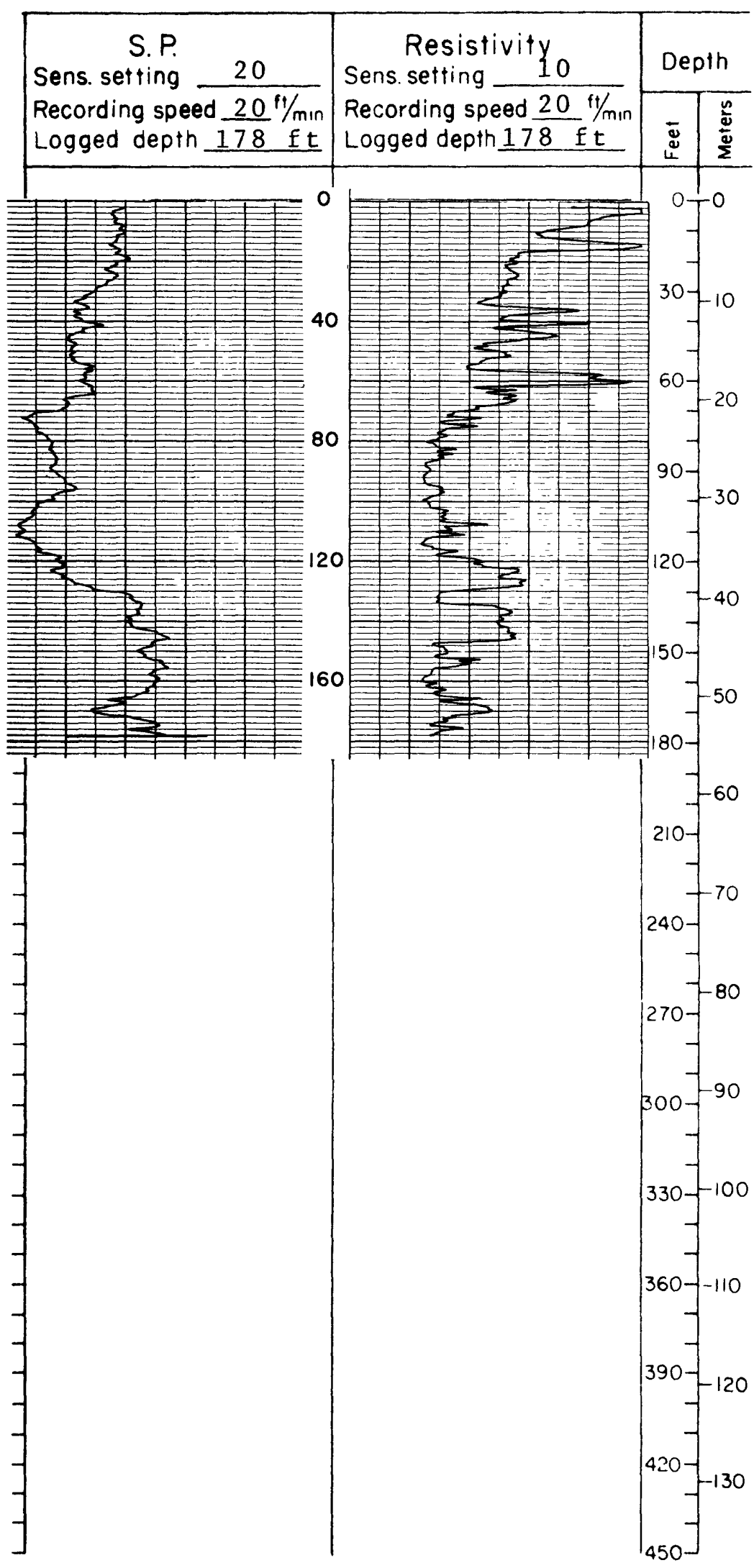


Hole no:Us -77164 Mop:Poverty Flat Eas tDote9/26/77 State:Montana County: Dawson Elev. $2532 \mathrm{ft}$ Location: T. $16 \mathrm{~N}, \mathrm{R} .54 \mathrm{E}, \mathrm{Sec} 12$, Tract BBDB Drilled depth: $200 \mathrm{ft}$ Meosured:800 ft $\mathrm{FNL} 750 \mathrm{ft} F \mathrm{FL}$ Hole size:4 3/4 in. Air $\square$ Water $\otimes$ Cored:Yes $\square$ No⿴囗十 Remarks:

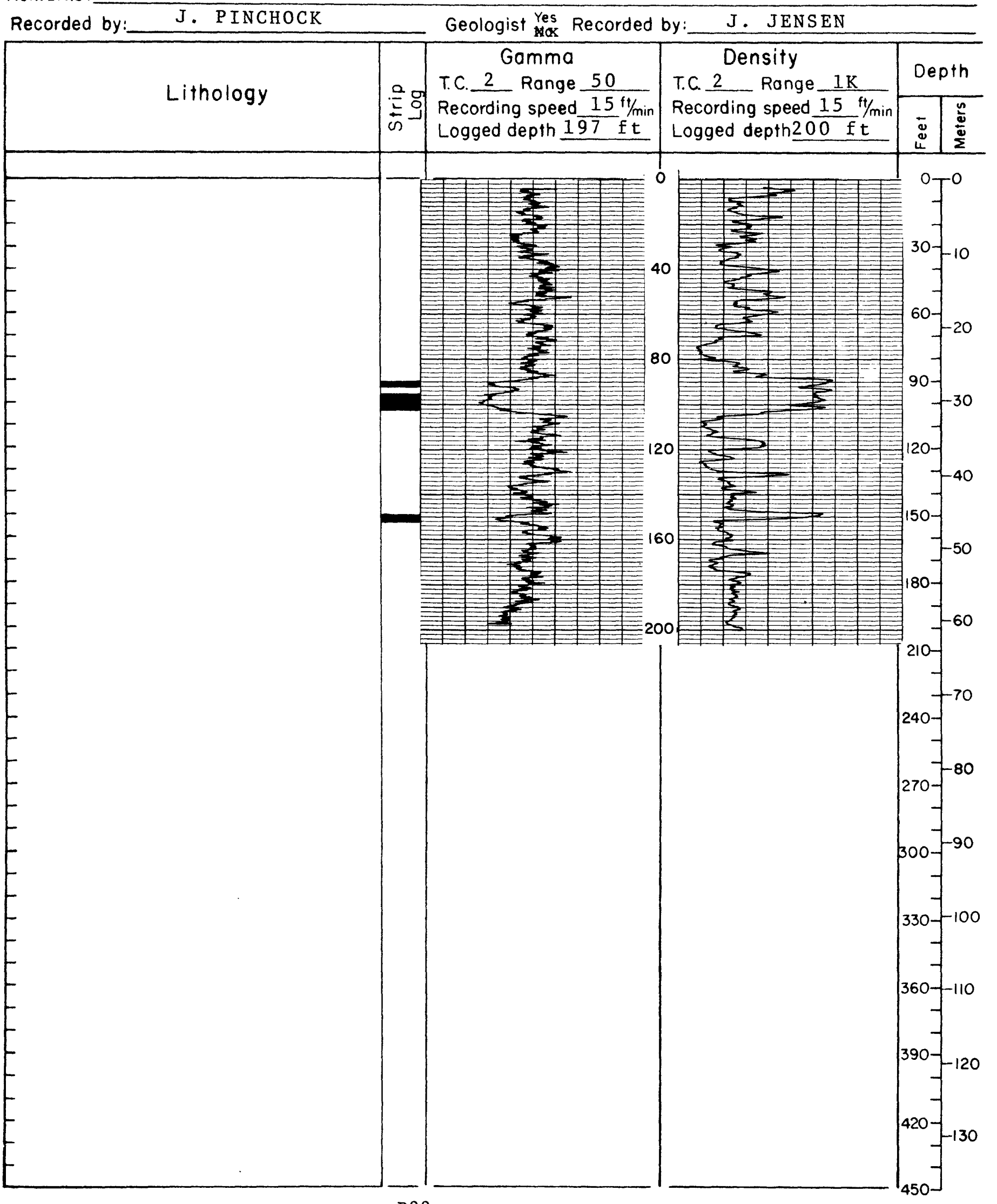


Hole number (continued)

$\underline{U S-77164}$

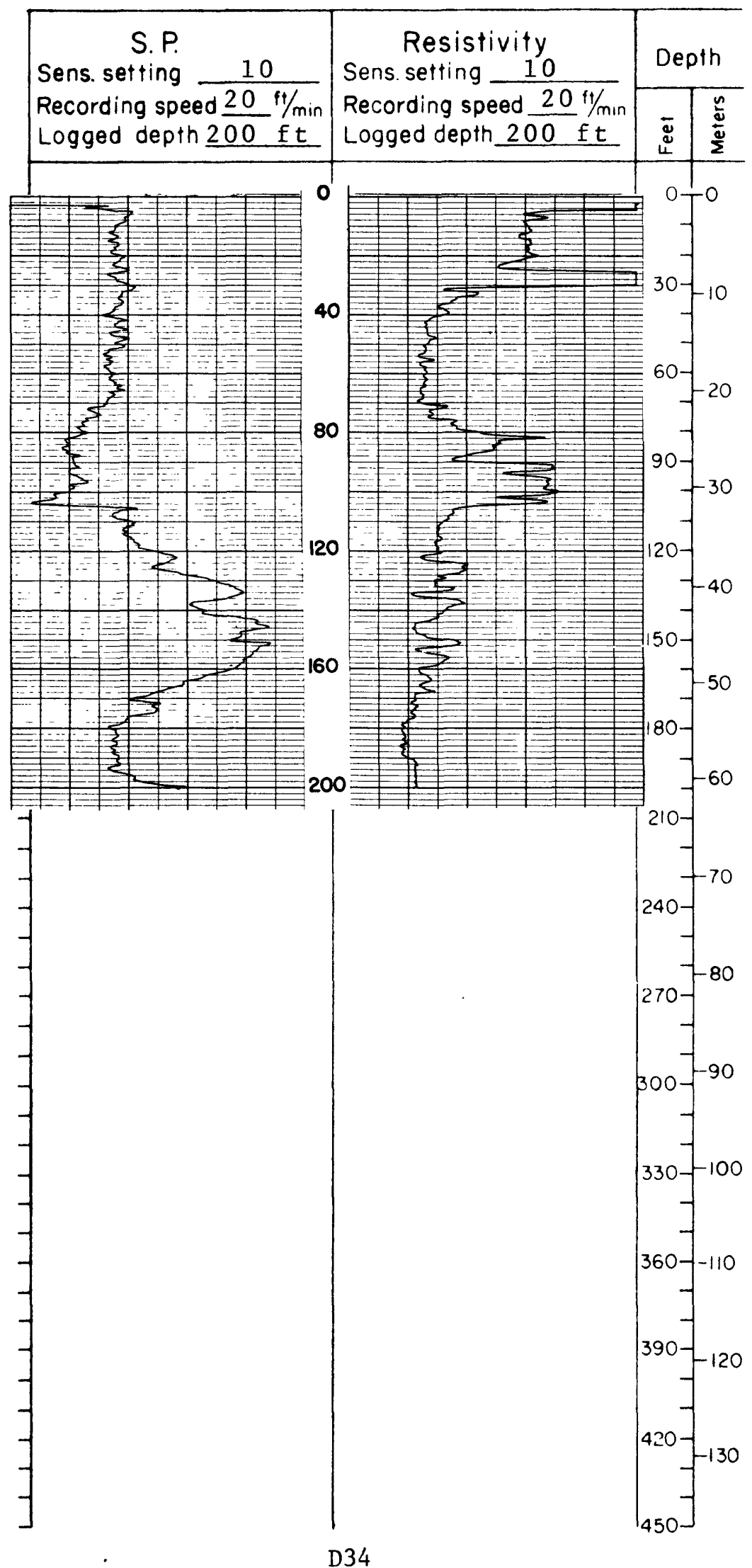




\section{MONTANA BUREAU OF MINES AND GEOLOGY \\ Montana College of Mineral Science and Technology}

Hole no: US -77165 Mop:Poverty Flat East Dote:9/27/77 State:MontanaCounty: Dawson Elev. $2480 \mathrm{ft}$ Locotion: T. $16 \mathrm{~N}, \mathrm{~N} .54 \mathrm{E}, \mathrm{Sec} .14$, Tract BCDD Drilled depth: $260 \mathrm{ft}$

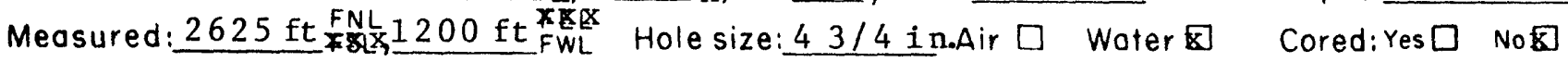
Remarks:

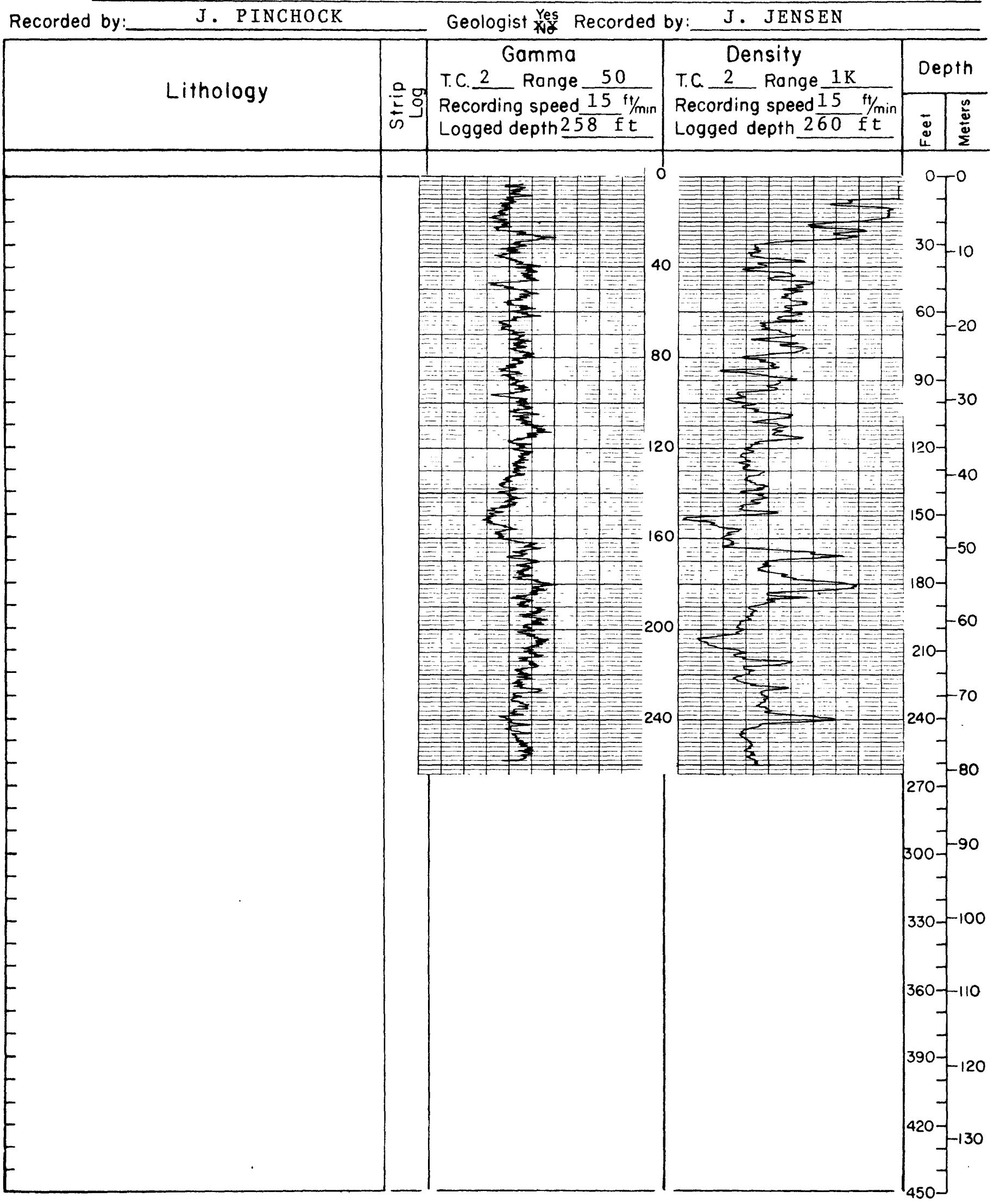


Hole number (continued)

$\underline{U S-77165}$

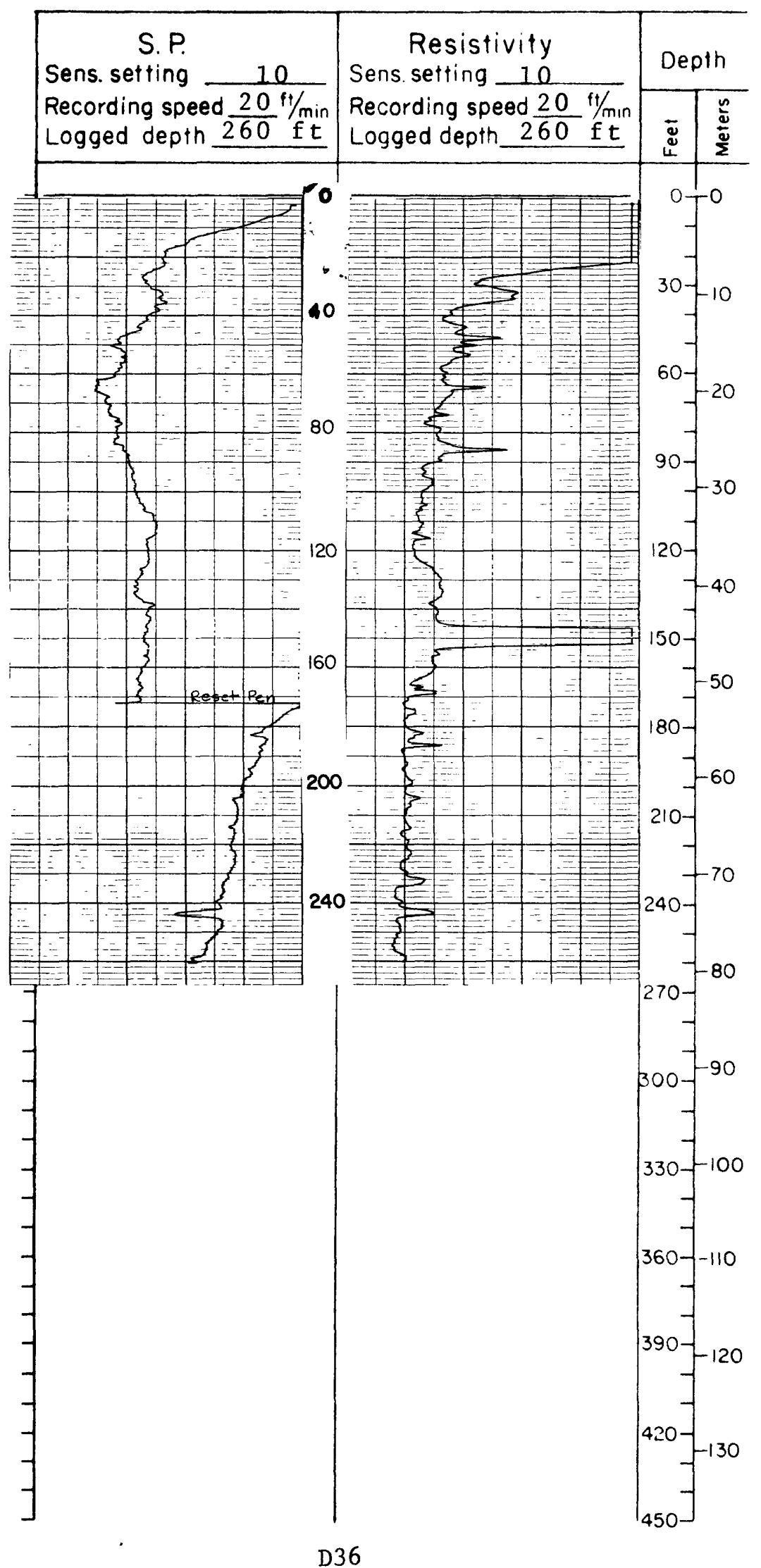




\section{MONTANA BUREAU OF MINES AND GEOLOGY \\ Montana College of Mineral Science and Technology}

Hole no:Ls-77166 Map:Stinking Coule eDote 9/27/77 State: MontanaCounty: Dawson Elev. $2555 \mathrm{ft}$ Locotion: T. 17 N. R. 53 W. Sec.12, Troct AAAA Drilled depth: $220 \mathrm{ft}$

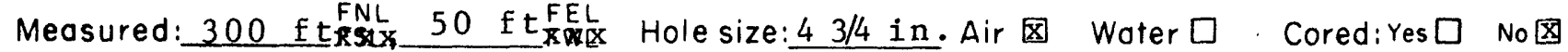
Remarks:

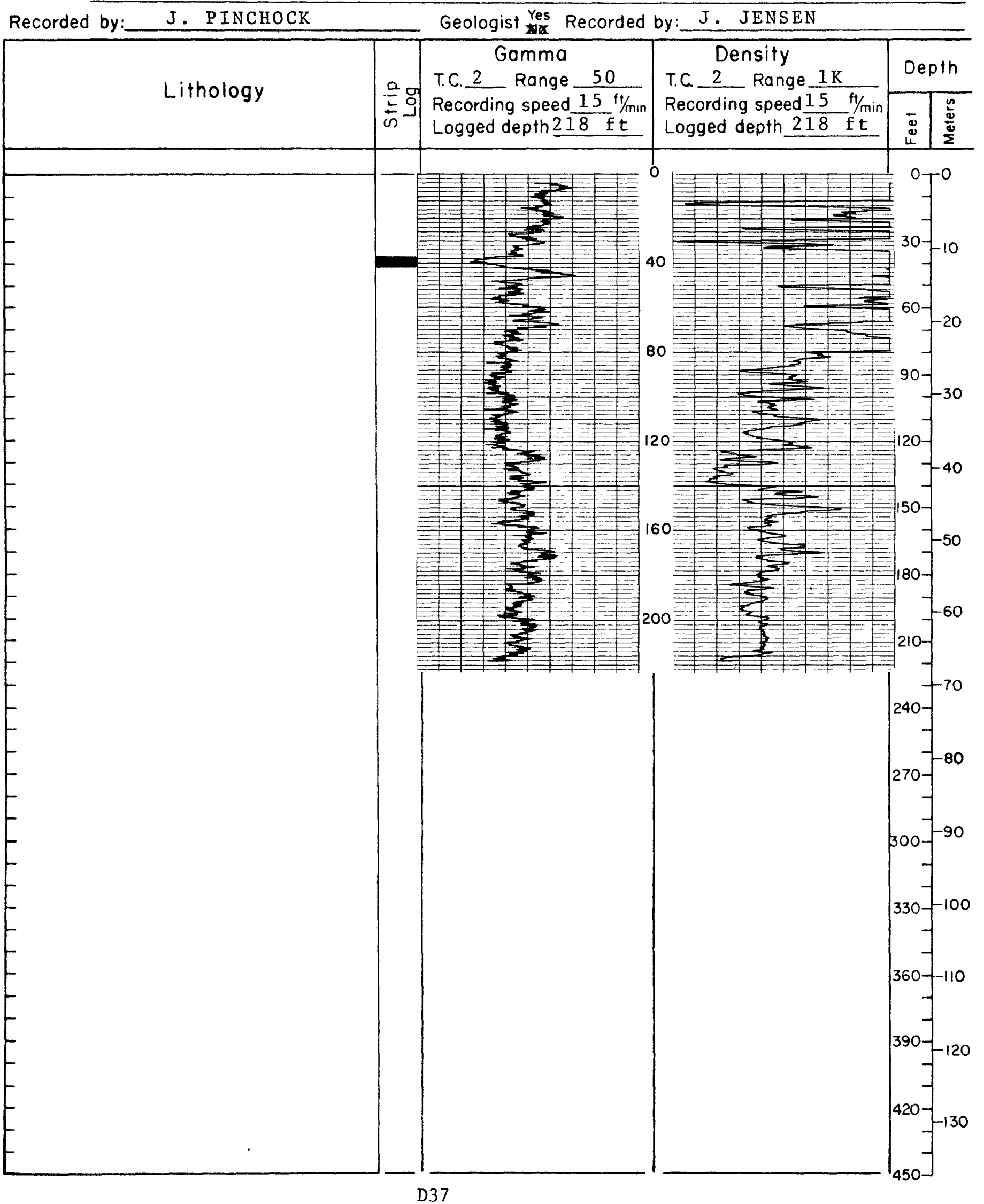


Hole number (continued)

$\underline{\text { US - } 77166}$

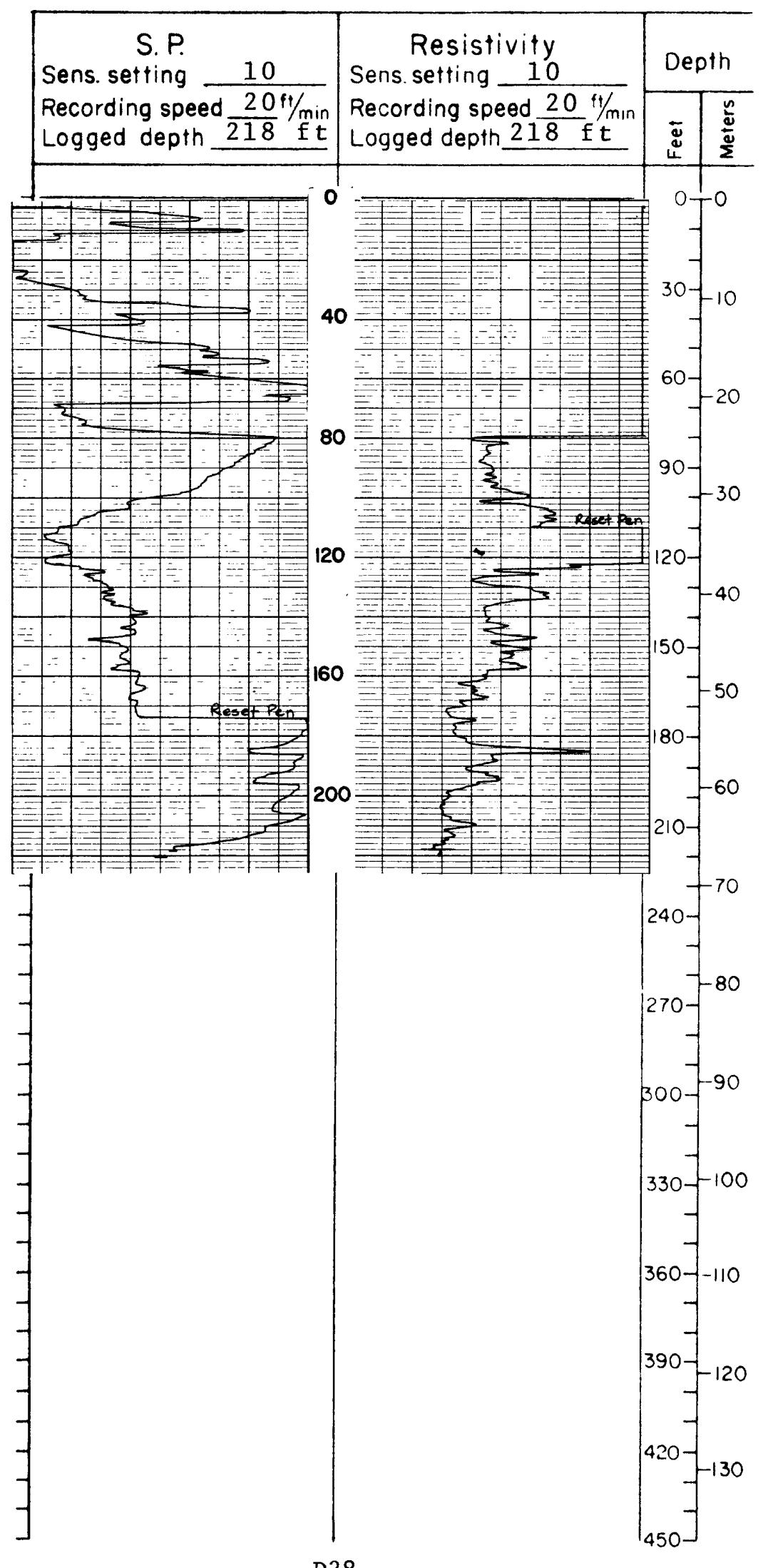


Hole no:US-77167 Mop:Stinking Coulee Date9/27/77 State:Montana County:_Dawson Elev.:2614 ft Location: T._18 $\mathrm{N}, \mathrm{R} .53 \mathrm{E}, \mathrm{Eec} .22$, Tract_BCCC Drilled depth: $600 \mathrm{ft}$

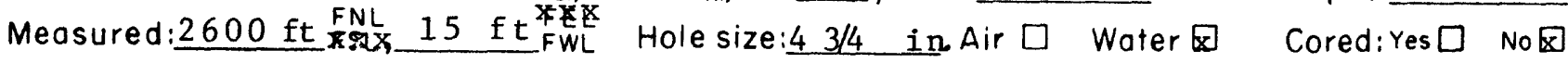

Remarks:

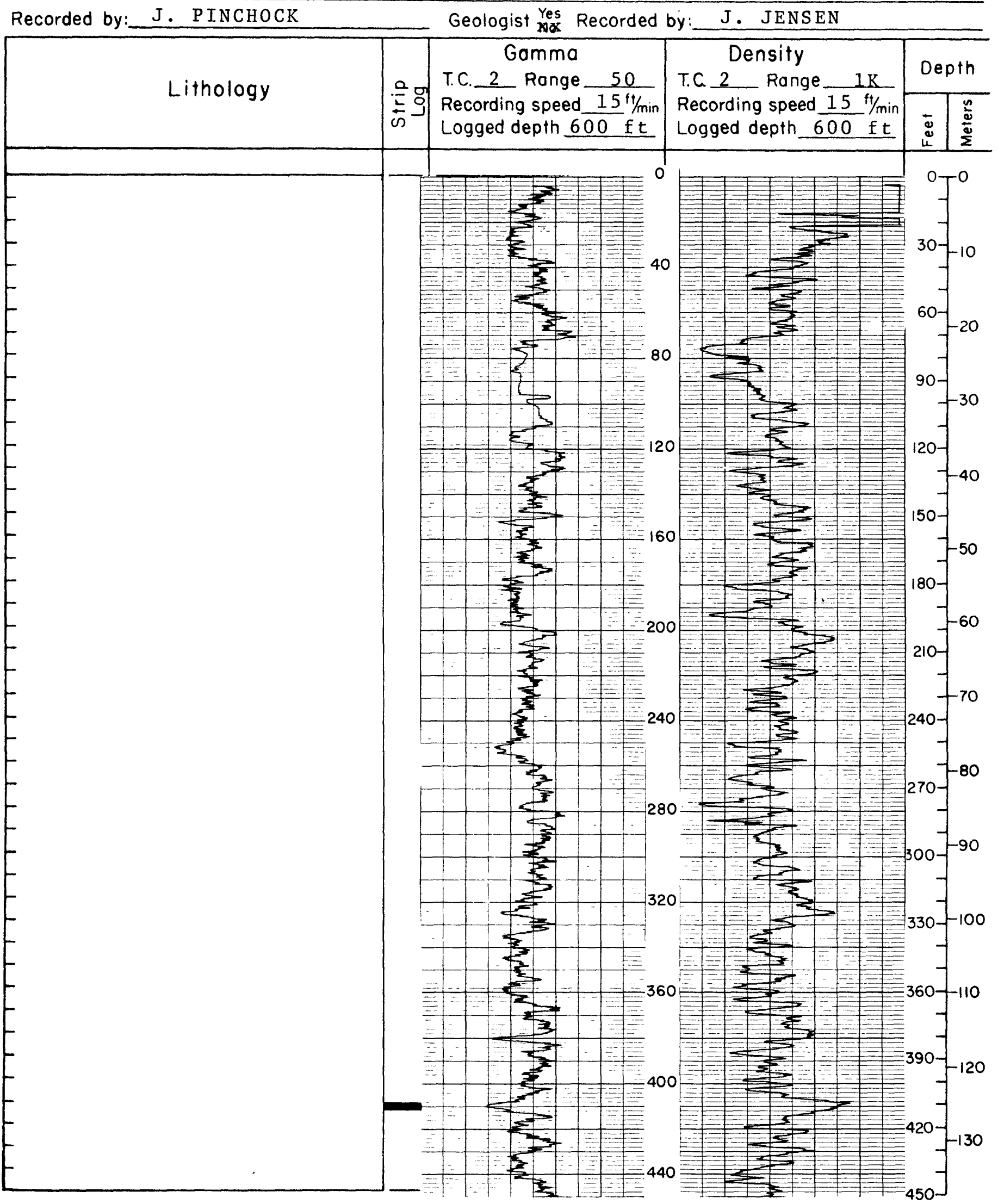


Hole number (continued)

US - 77167

Lithology

\begin{tabular}{|l|l|}
\hline Gamma \\
co & $\begin{array}{l}\text { T.C. } 2 \text { Range } \frac{50}{\text { Recording speed } 15 \mathrm{fl} / \mathrm{min}} \\
\text { Logged depth } 600 \mathrm{ft}\end{array}$ \\
\hline
\end{tabular}

T.C. $2^{\text {Density }}$

Recording speed $15 \mathrm{ft}$

Depth
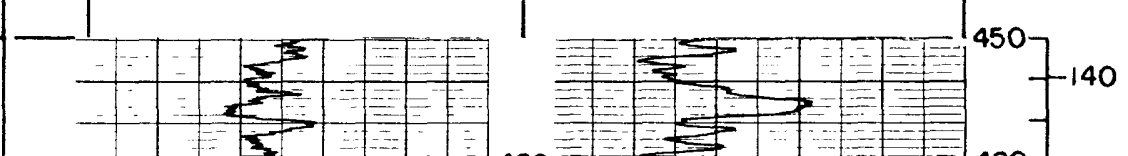

$\therefore-14$

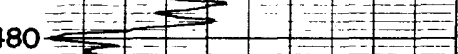

$480-$

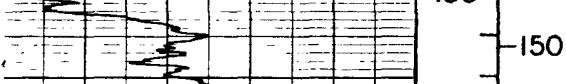

票

520

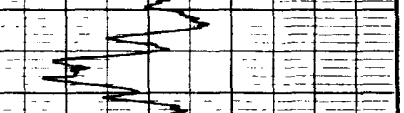

$510-$
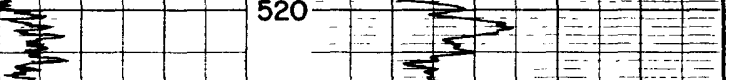

$-160$

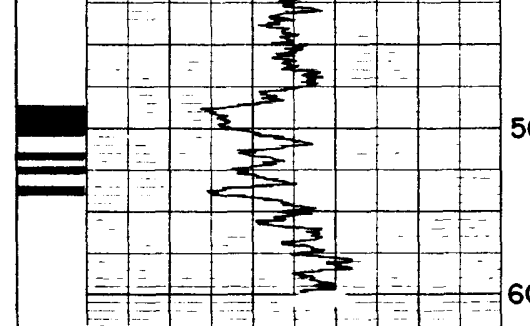

$\mid$
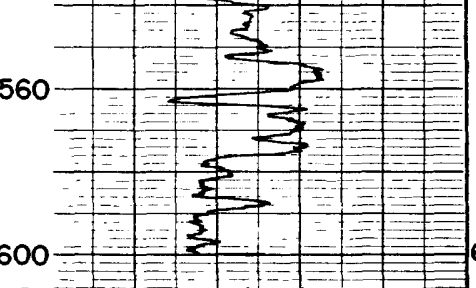

$-170$

$570-$

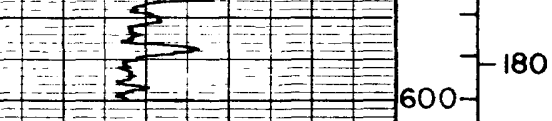

I

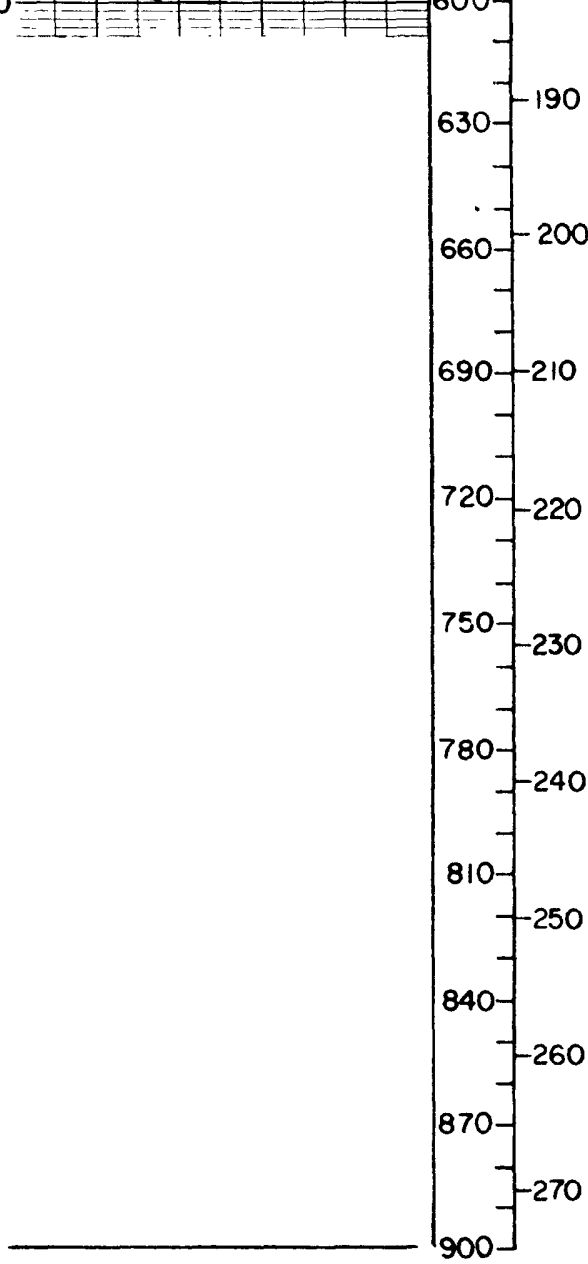


Hole number (continued)

$\underline{U S-77167}$

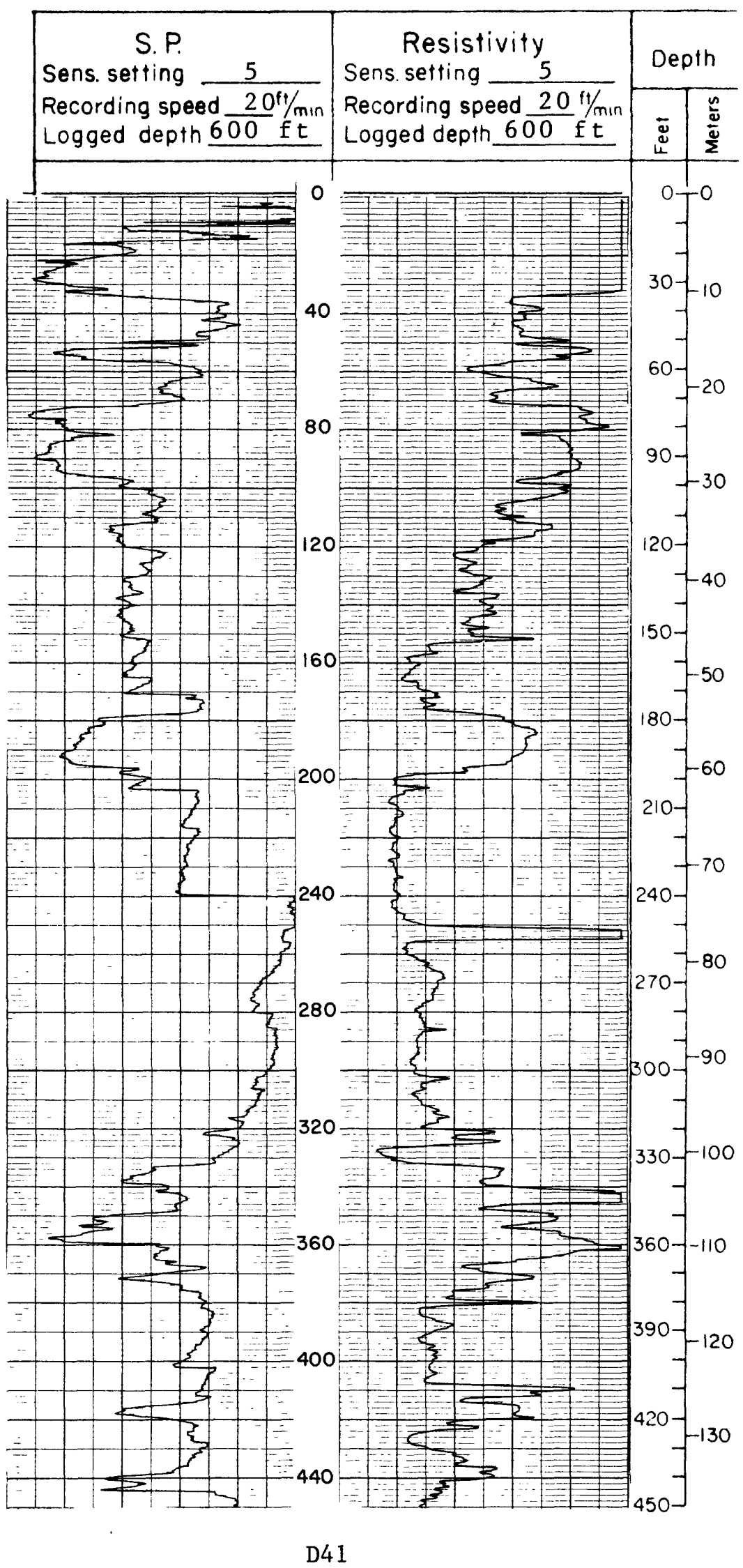


Hole number (continued)

$\underline{U S-77167}$

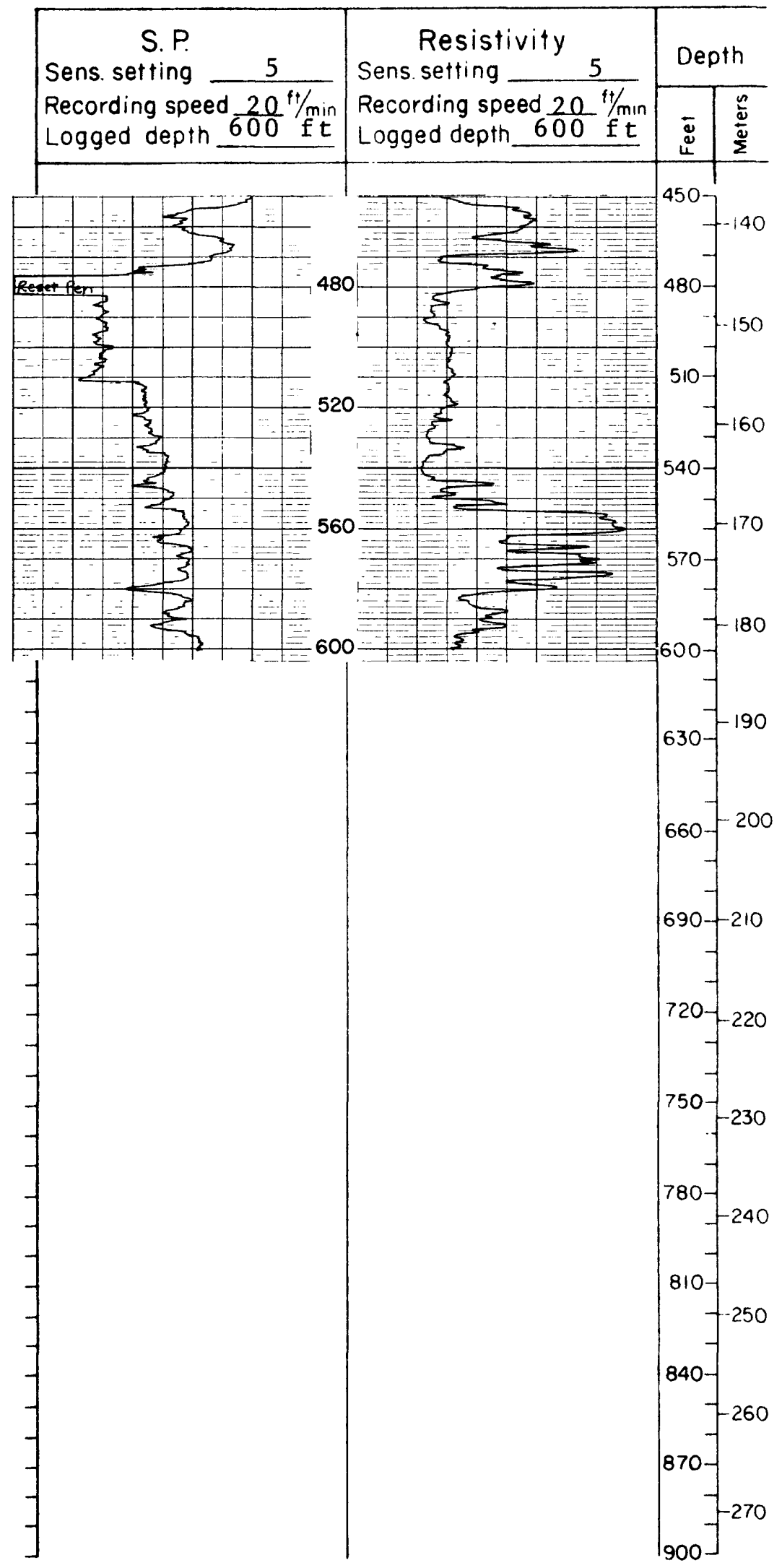




\section{MONTANA BUREAU OF MINES AND GEOLOGY \\ Montana College of Mineral Science and Technology}

Hole no:US-77168 Map: BLM-NE 31

Dote $9 / 28 / 77$ State:Montana County: Dawson

Elev.:2634 ft Location: T. 18 N R. 52 E Sec. 12 , Tract CCCC Orilled depth: $500 \mathrm{ft}$

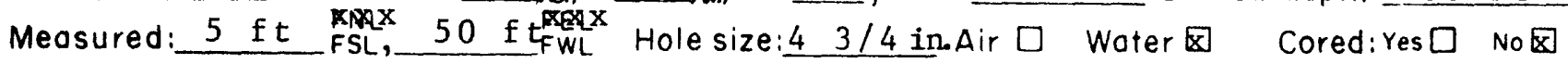
Remorks: Density $\log$ is $3 \mathrm{ft}$ high on chart

Recorded by:

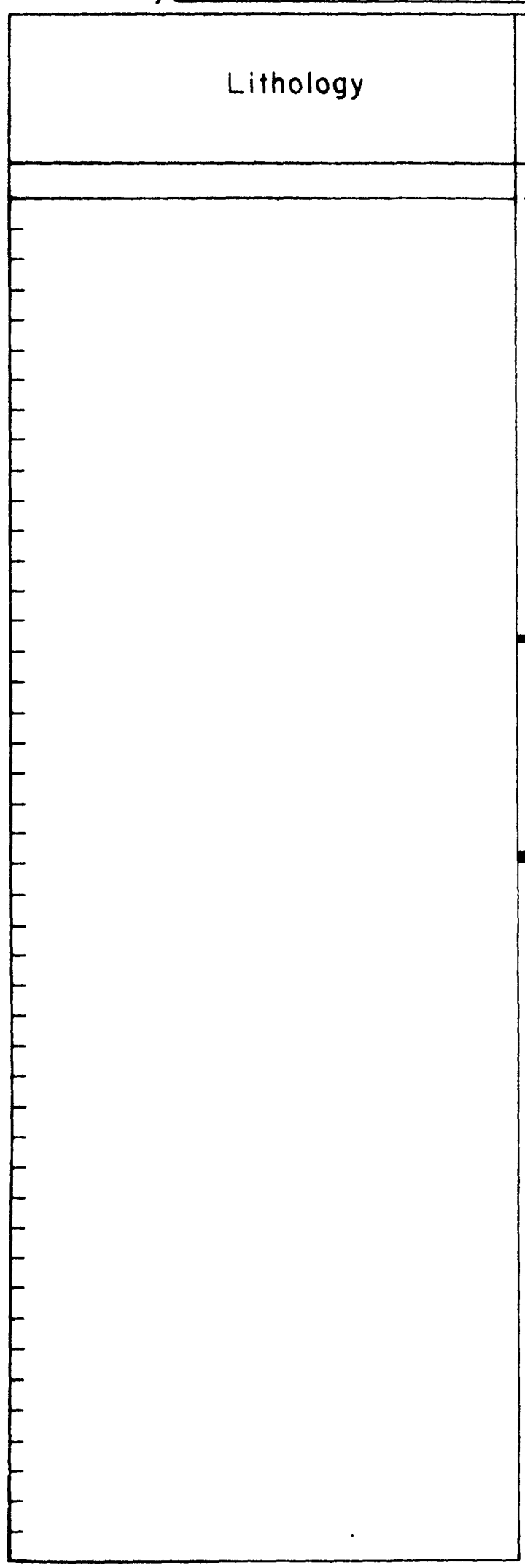

Geologist Yes Recorded by: J. JENSEN

\begin{tabular}{|c|c|c|}
\hline & Gomma & Density \\
\hline & T.C. 2 Range $\quad 50$ & - Range $1 \mathrm{~K}$ \\
\hline & $\begin{array}{l}\text { Recording speed } 15 \mathrm{ft} / \mathrm{min} \\
\text { Logged depth } \overline{500} \mathrm{ft}\end{array}$ & $\begin{array}{l}\text { Recording speed } 15 \mathrm{ft} / \mathrm{min} \\
\text { Logged depth } 5 \overline{00 \mathrm{ft}}\end{array}$ \\
\hline
\end{tabular}

Depth

\begin{tabular}{|c|}
\hline 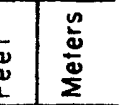 \\
\hline
\end{tabular}

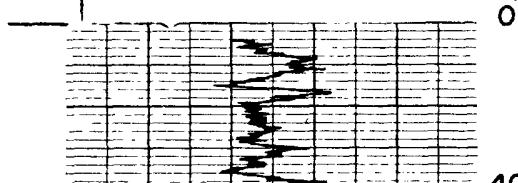

40

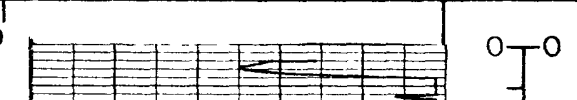

40

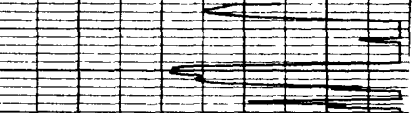

$+\div+2$

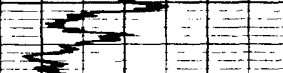

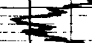

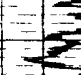

5

1

$\div=$

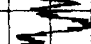

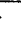

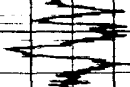

$\sum_{0}^{2}$
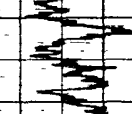

$=$
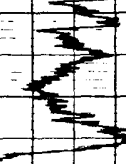

$+$

$\Rightarrow$

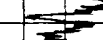

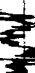

$\Rightarrow$

$=$

2

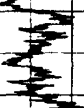

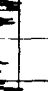

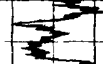

I

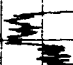

$=$

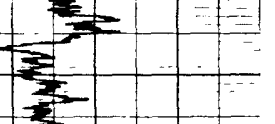

$\div$

$\frac{5}{5}$

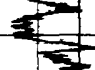

E

440

$\frac{1}{2}=$

400

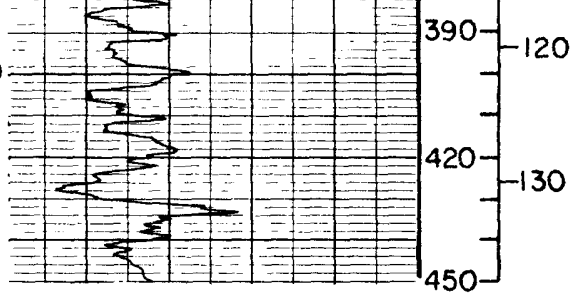

D 43 
Hole number (continued)

US- 77168

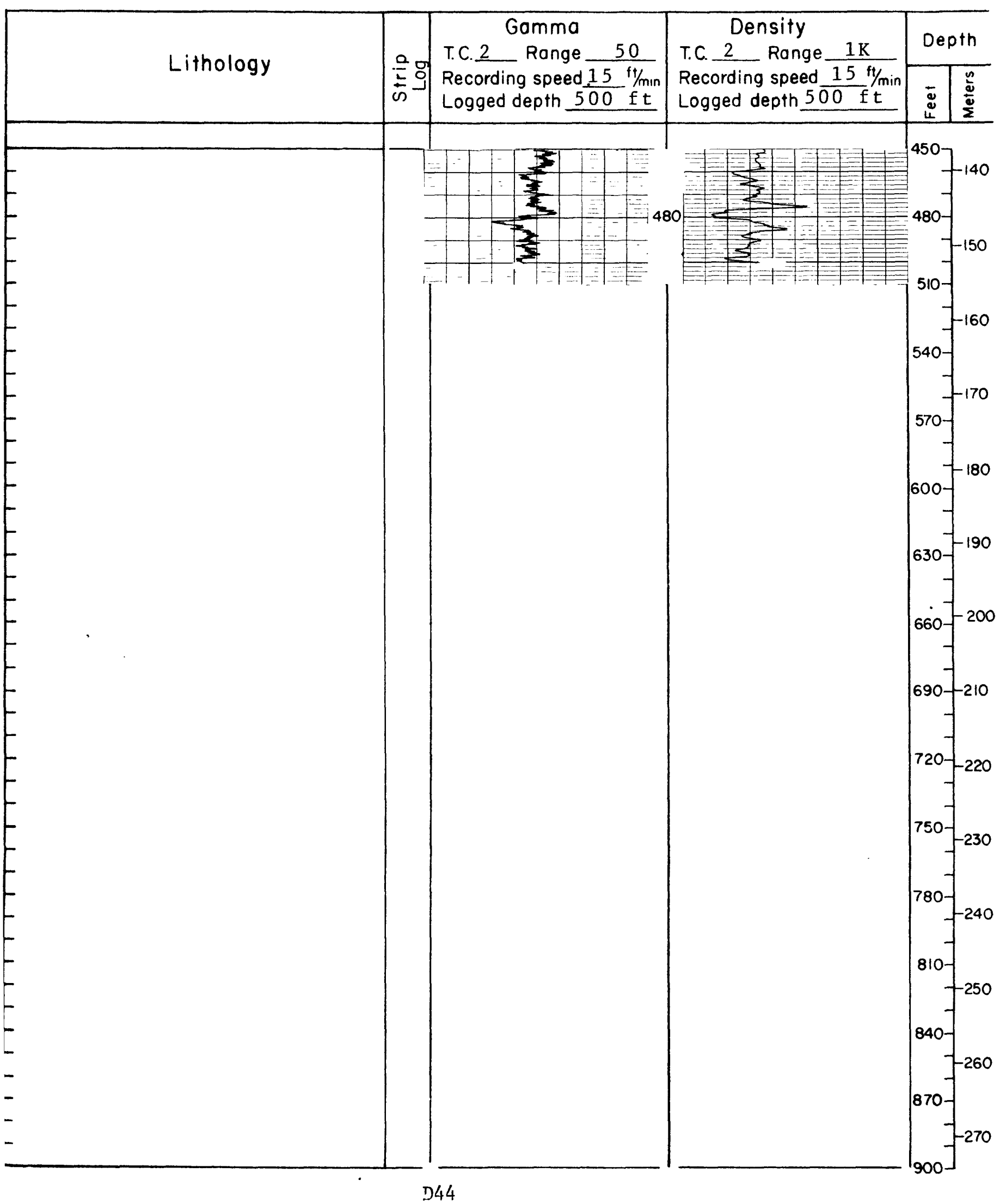


Hole number (continued)

$\underline{U S-77168}$

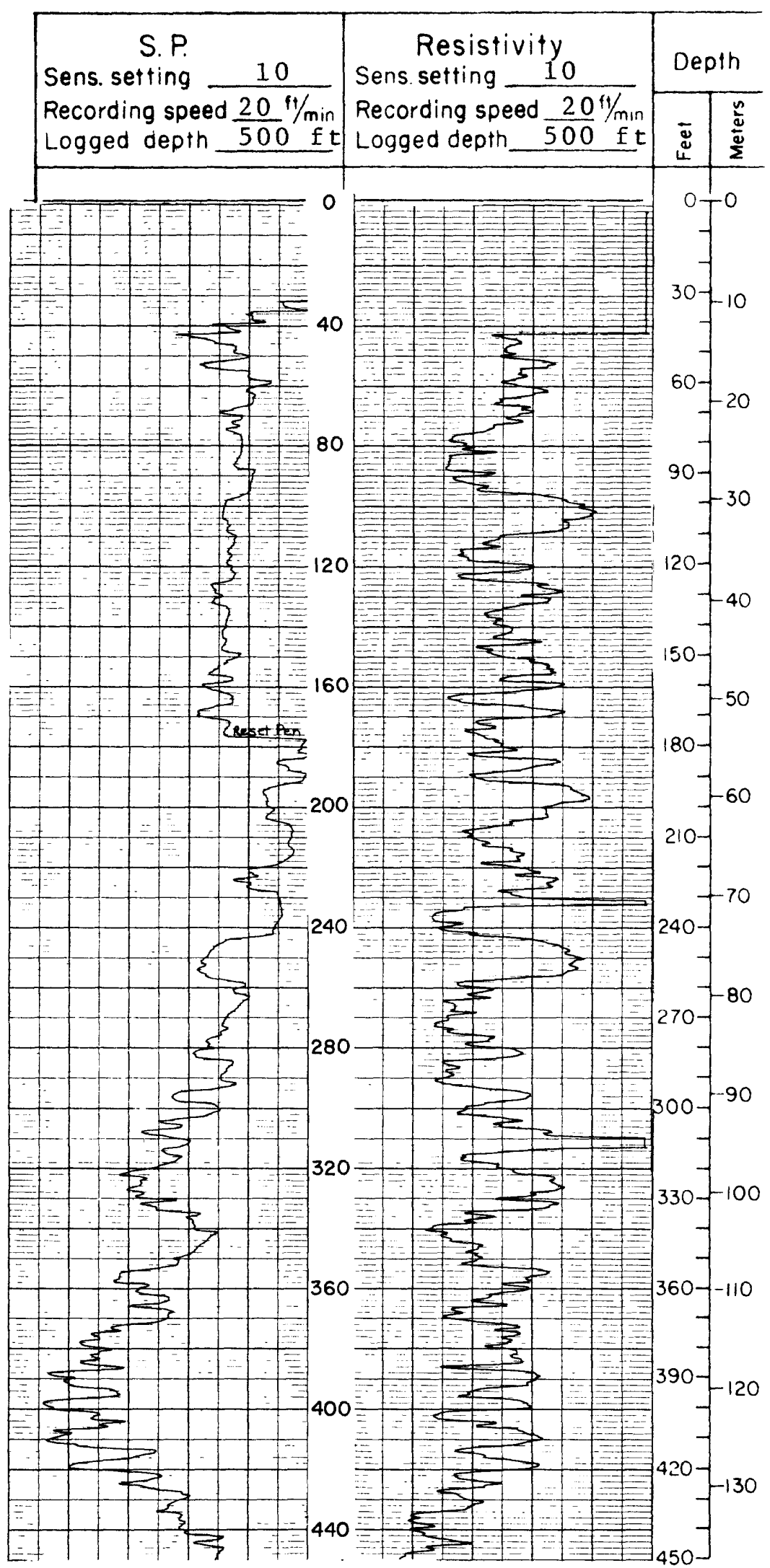


Hole number (continued)

$\underline{U S-77168}$

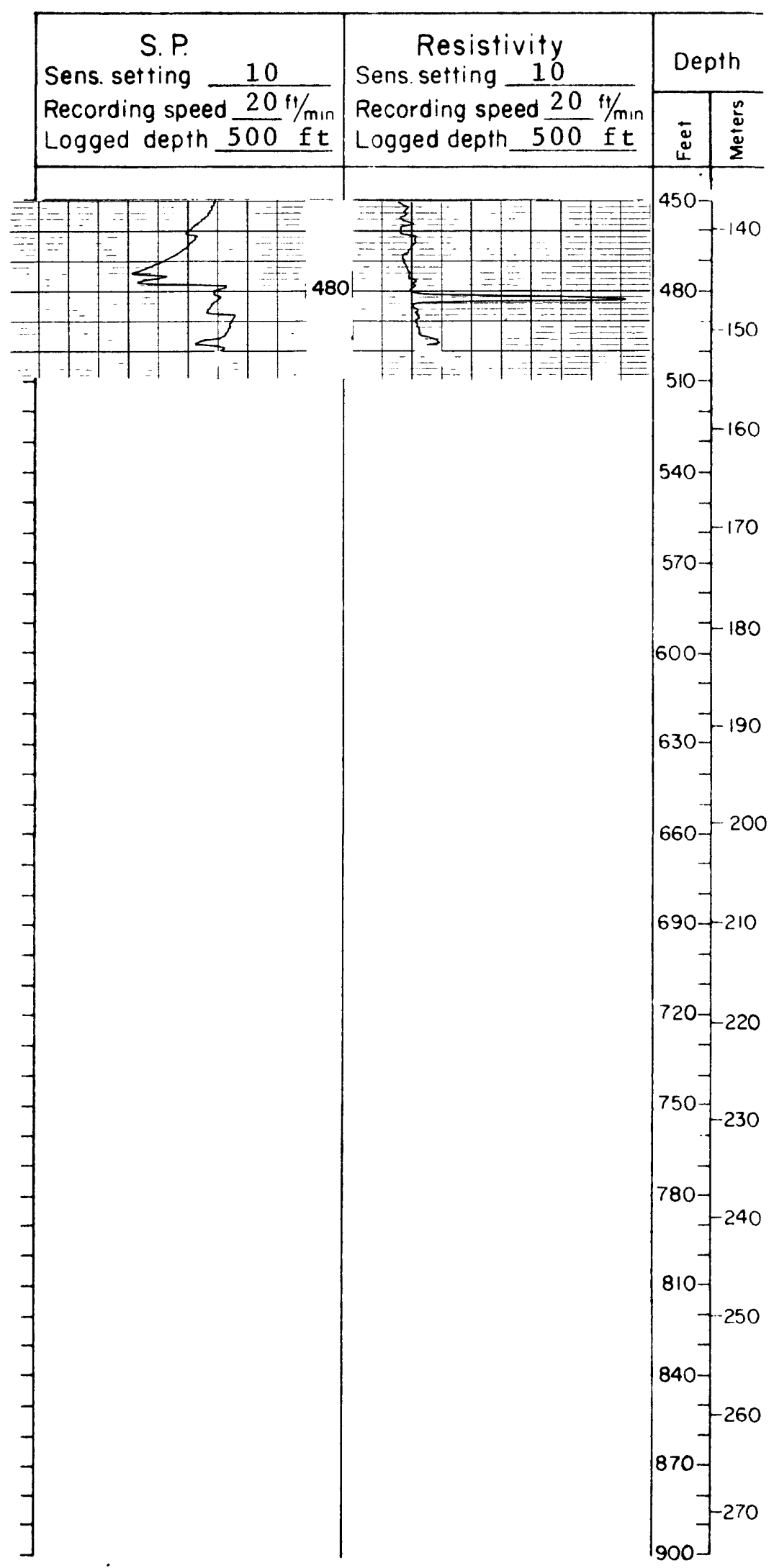

D46 
Montana College of Mineral Science and Technology

Hole nous-77169 Map:Poverty Flat West Dote9/29/77 Stateintana County: Dawson Elev: $2760 \mathrm{ft}$ Location: T. $17 \stackrel{\mathrm{N}}{\mathrm{S}}, \mathrm{R} .53 \mathrm{E}$, Sec. 28, Tract DCBC Drilled depth: $500 \mathrm{ft}$ Measured:680 ft

Remarks:

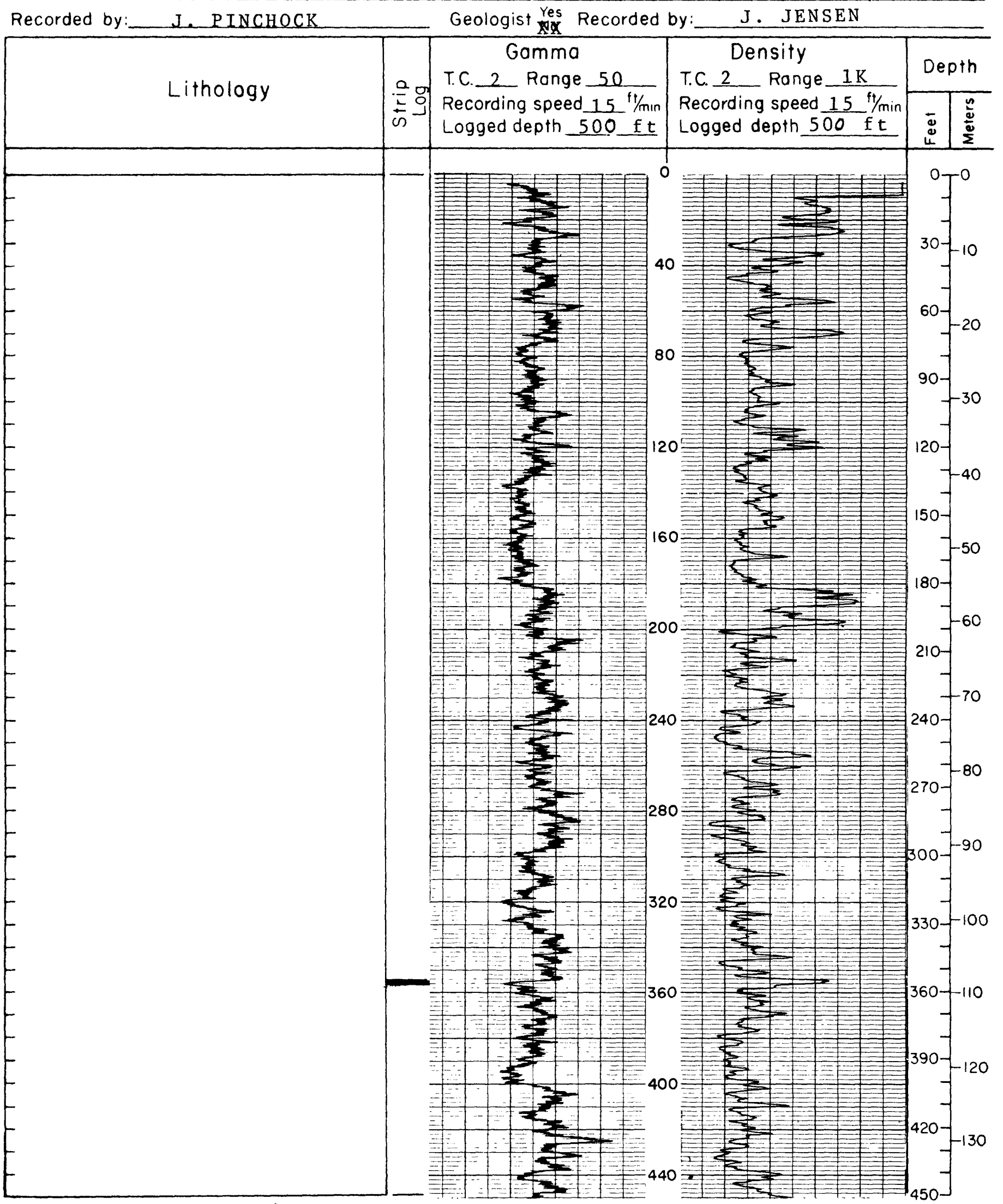


Hole number (continued)

US -77169

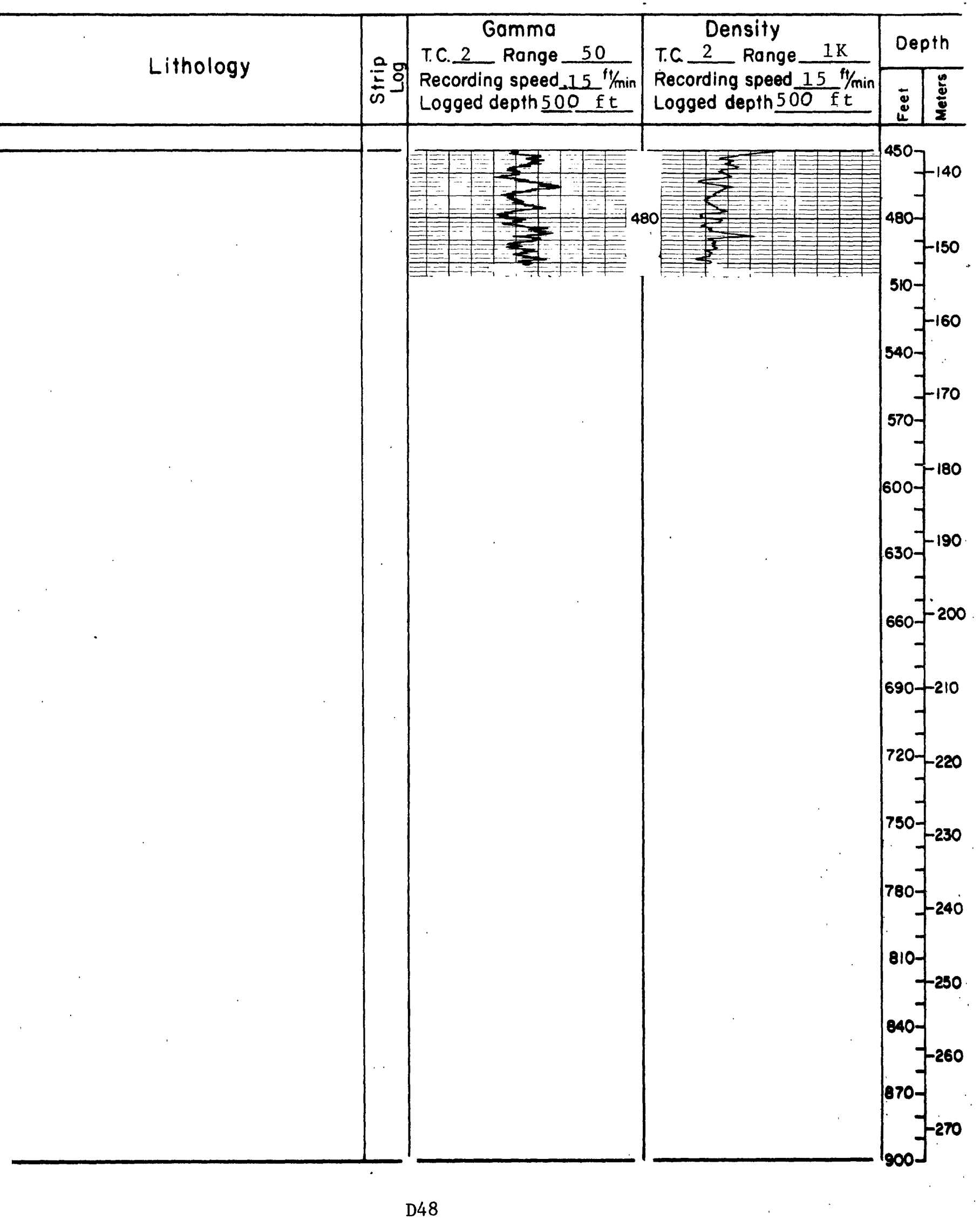


Hole number (continued)

$\underline{U S-77169}$

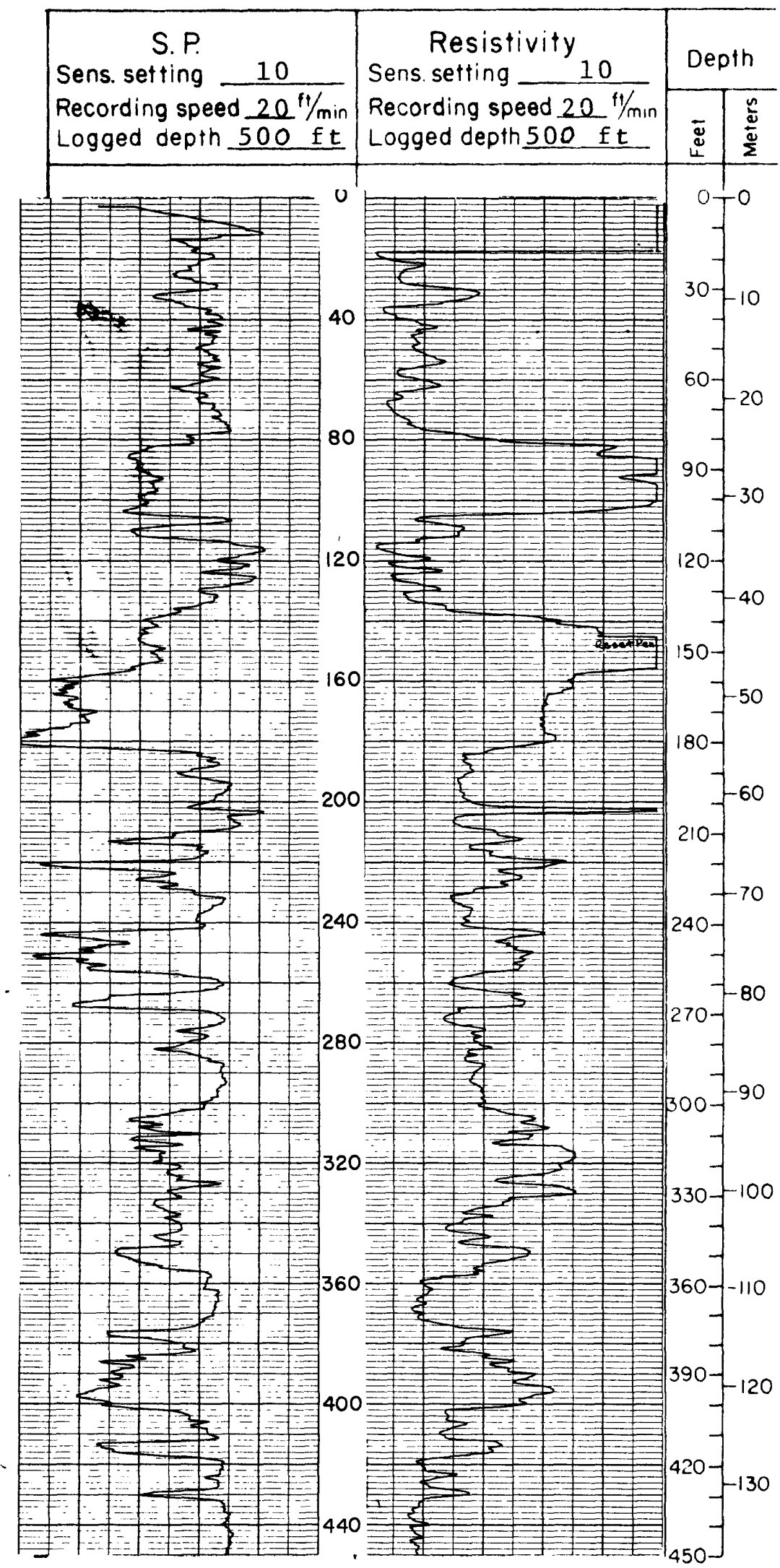


Hole number (continued)

$\underline{U S-77169}$

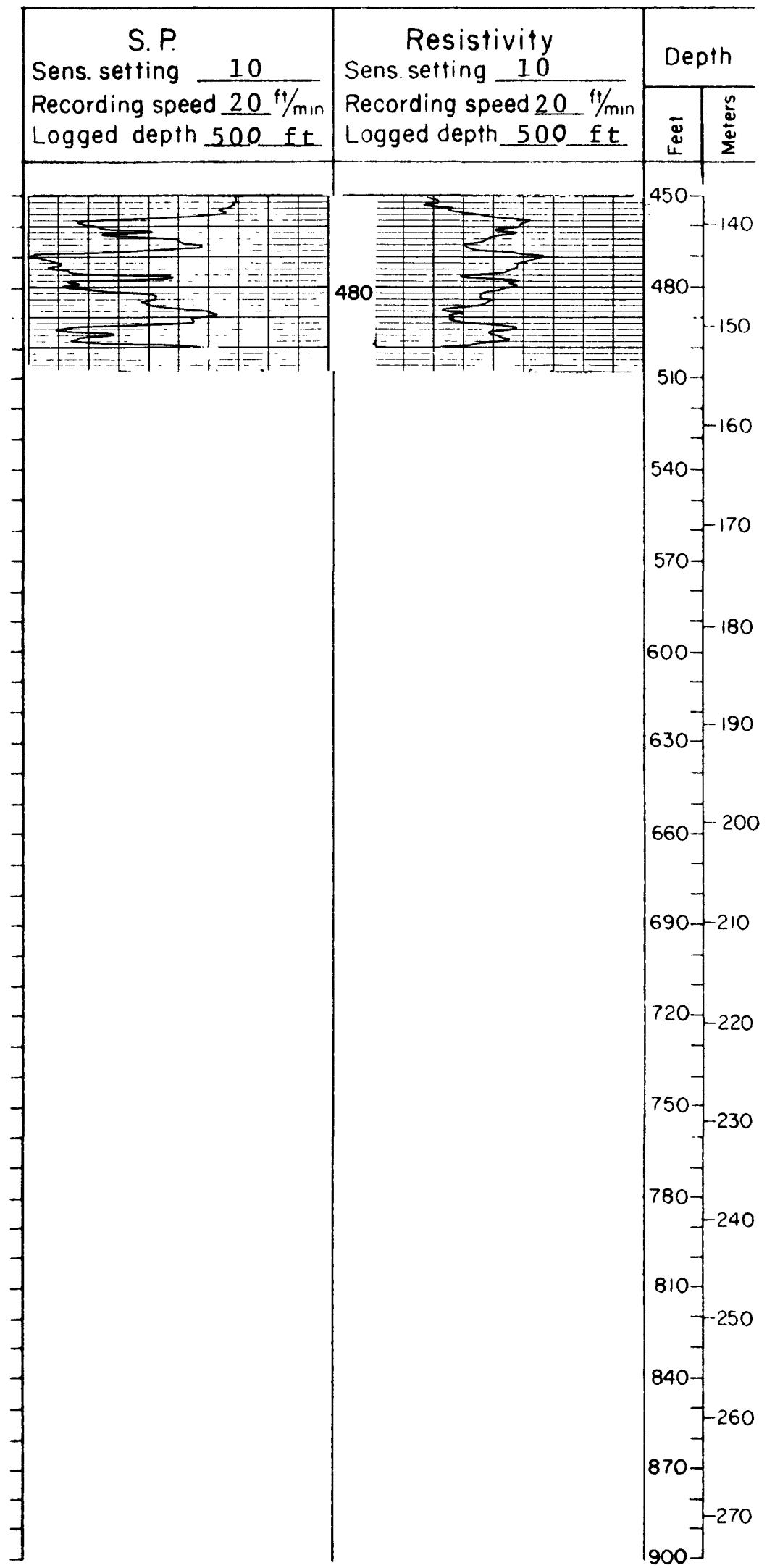


Montana College of Mineral Science and Technology

Hole no:US- 77170 Map:Stinking CouleeDote10/5/77 StateMontana County: Dawson Elev. $2502 \mathrm{ft}$ Location:T. 17 N. R. 53 W. Sec. 8, Tract_AAAB Drilled depth: $502 \mathrm{ft}$

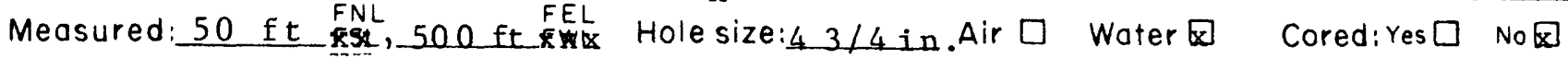
Remorks:

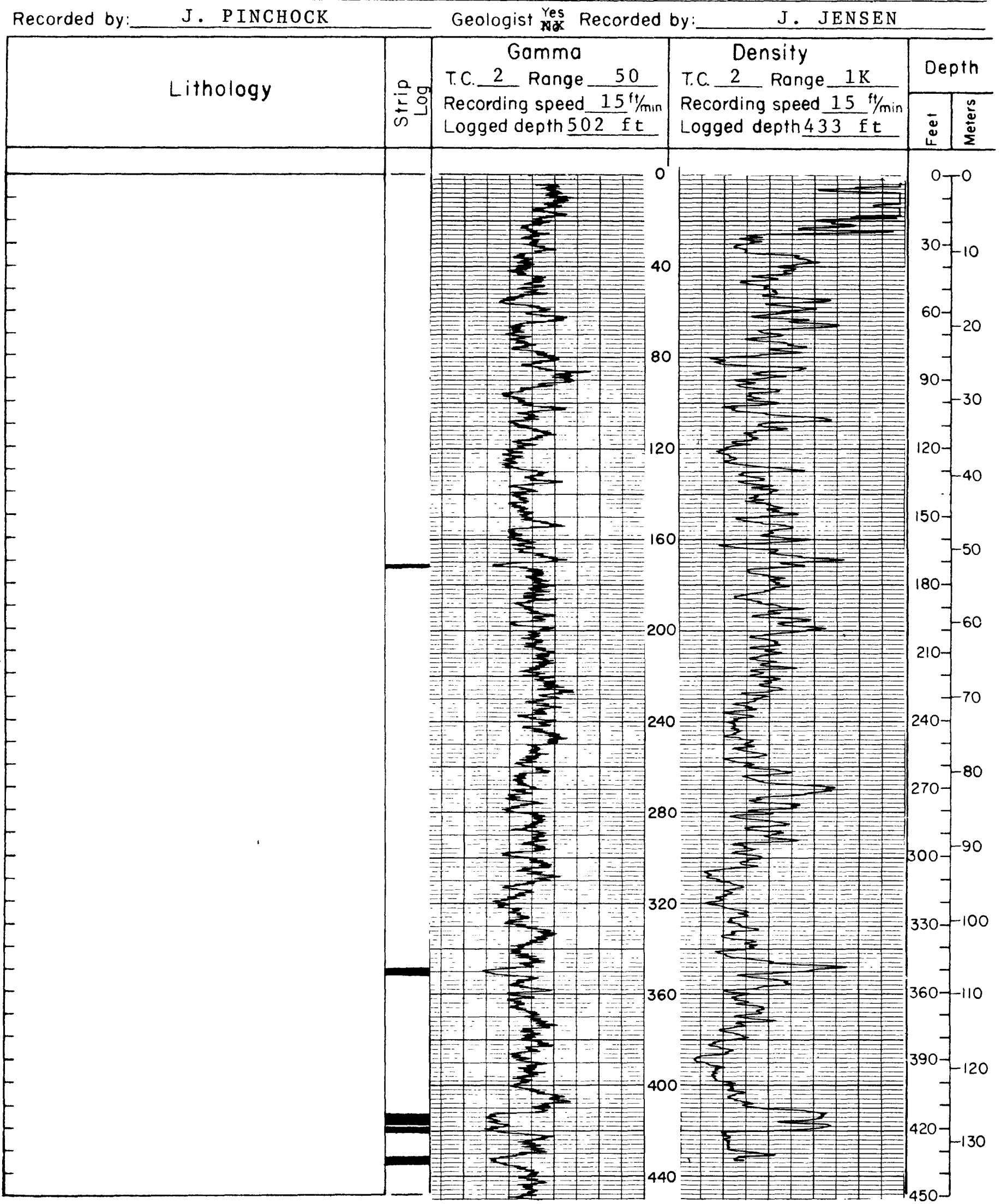


Hole number (continued)

$\underline{U S}-77170$

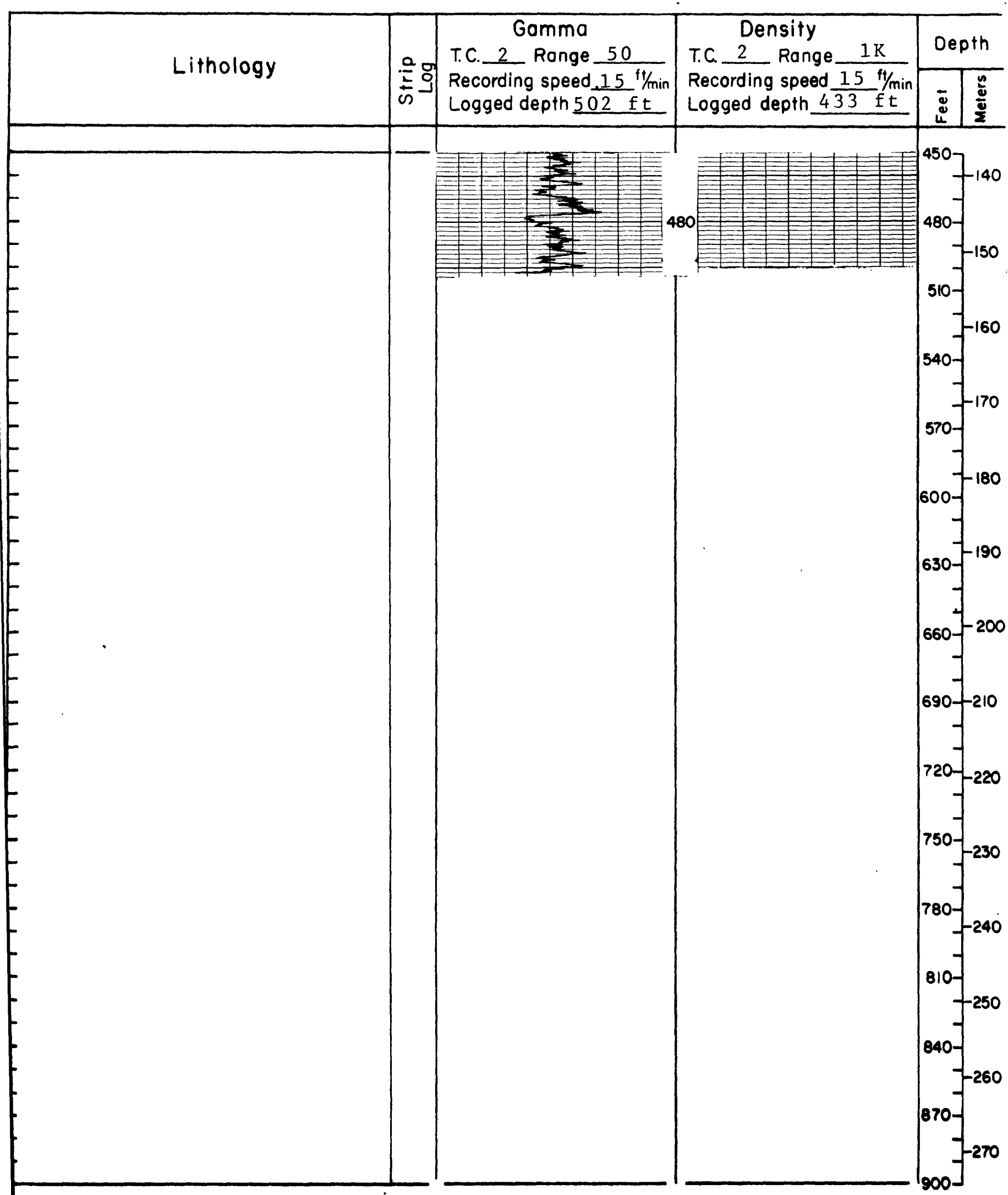


Hole number (continued)

$\underline{U S-77170}$

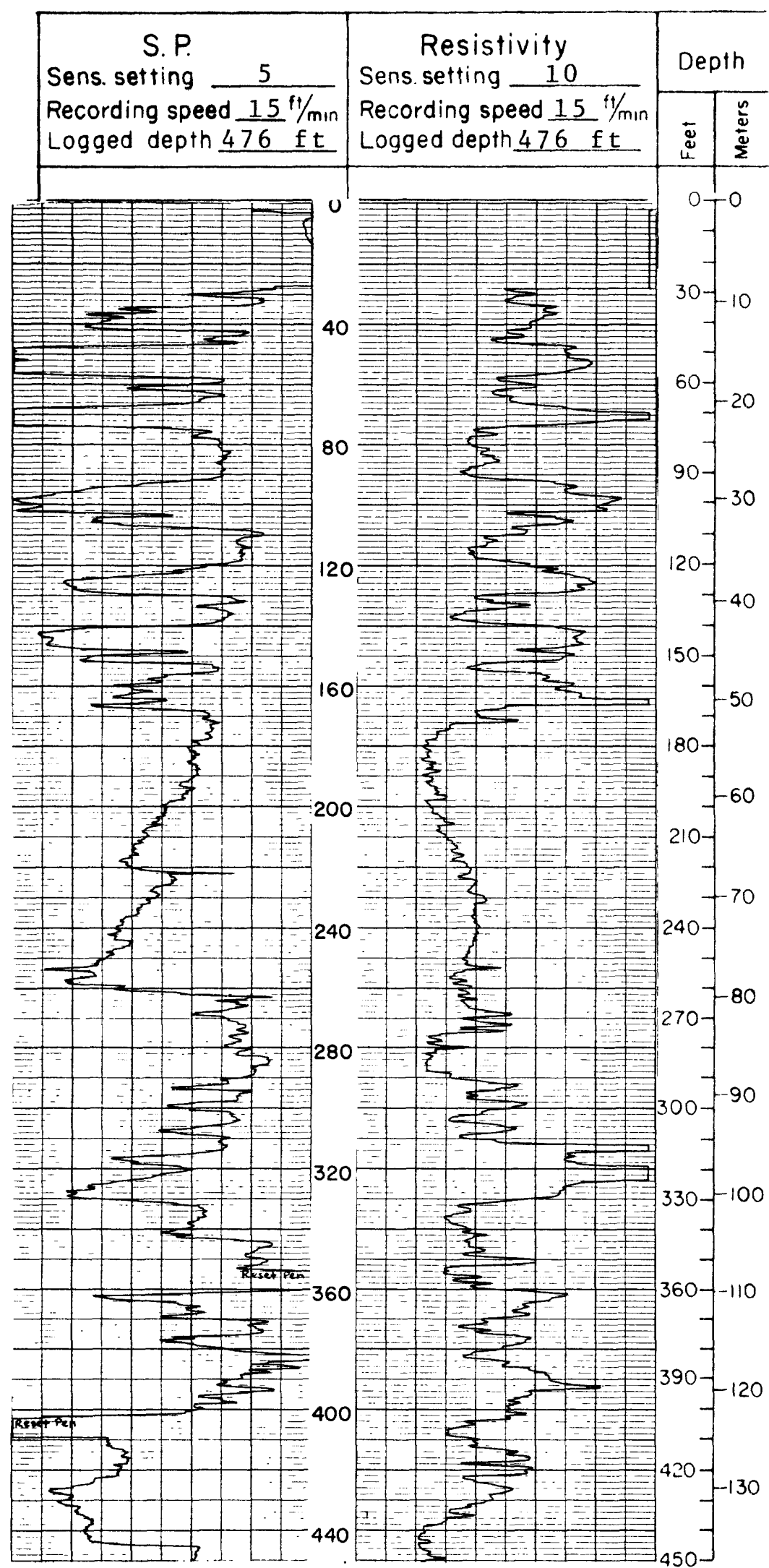


Hole number (continued)

$\underline{U S-77170}$

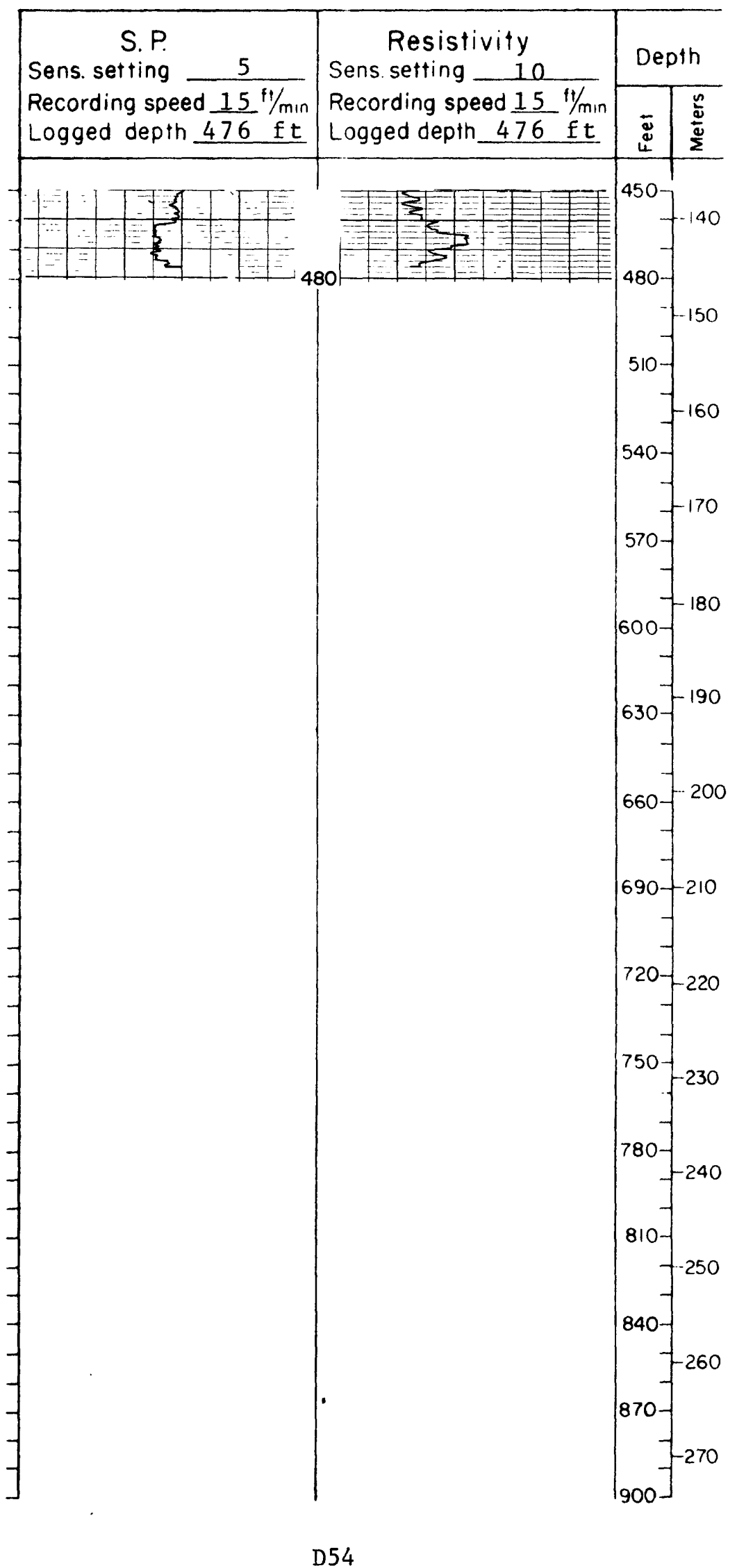


Hole no:US -77171 MOP:BLM-NE39 Dotel $0 / 6 / 7$ State:Mont ana County: Dawson Elev.: $2671 \mathrm{ft}$ Location: T. 17 N. R. 52 E. Sec. 20 , Troct_CCCC Drilled depth: $582 \mathrm{ft}$

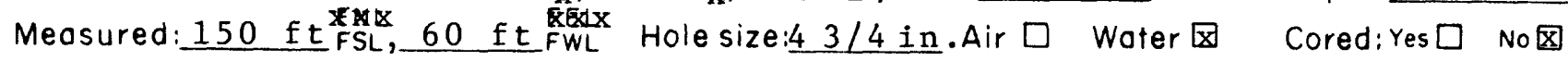

Remarks:

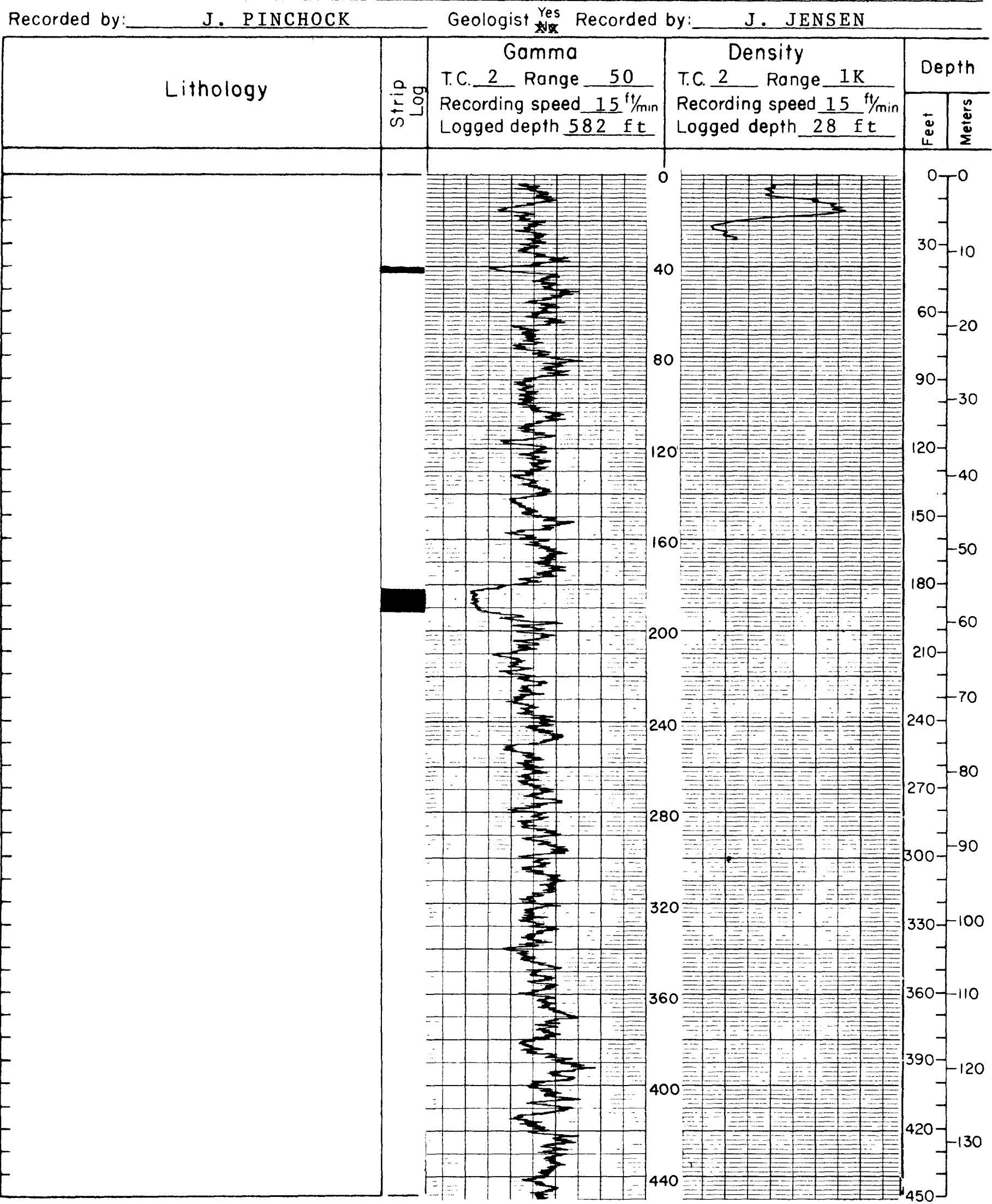


Hole number (continued)

US - 77171

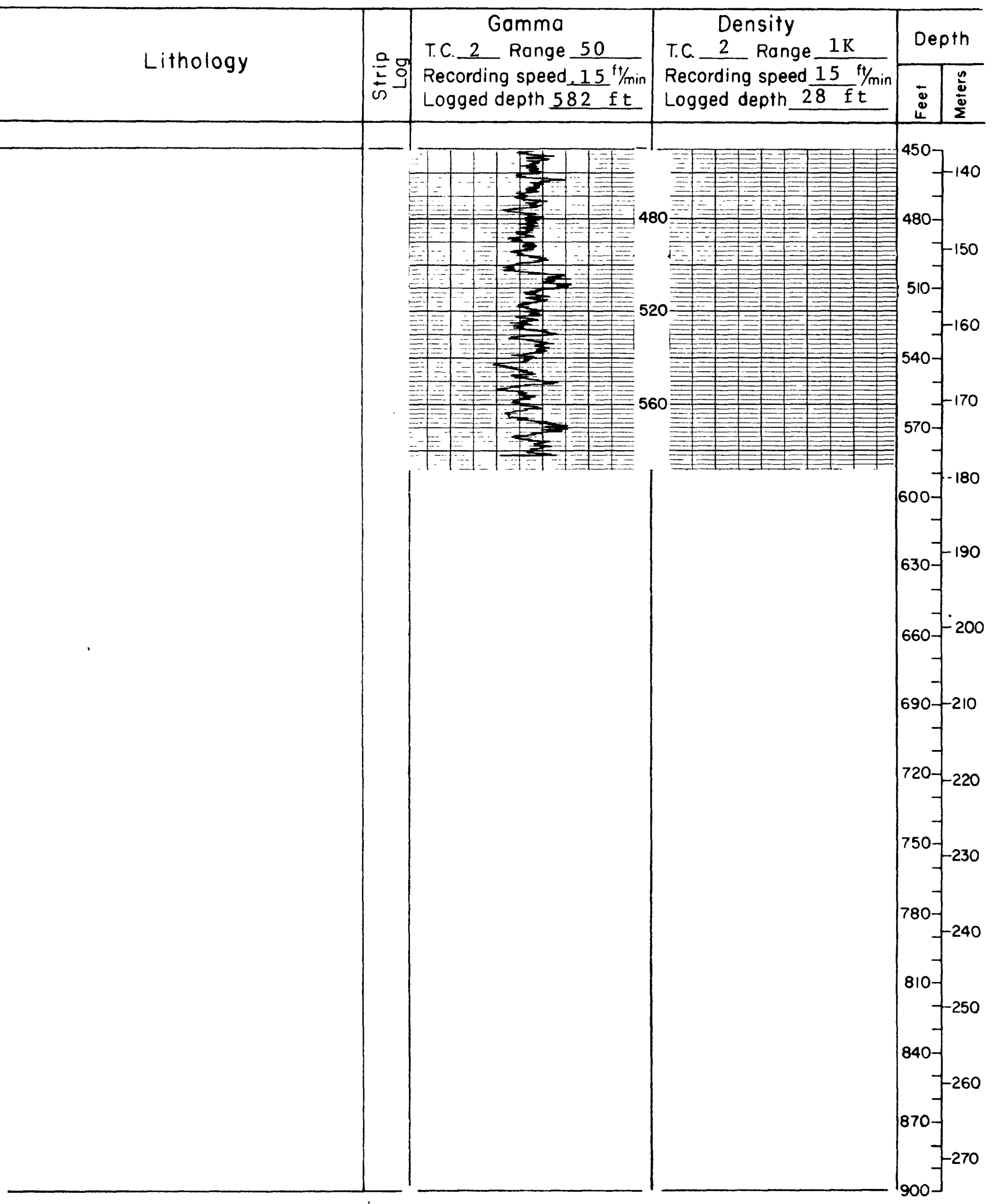


Hole no:US -77172 Map:_BLM-NE39.

Date10/7/7 State Montana County: Dawson

Elev.: Locotion: T. 16 N. R. 52 E. Sec. 10 , Tract B B BC Drilled depth: $572 \mathrm{ft}$

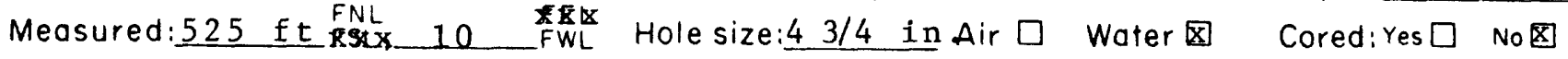
Remarks:

Recorded by: J. PINCHOCK Geologist Yes Recorded by:

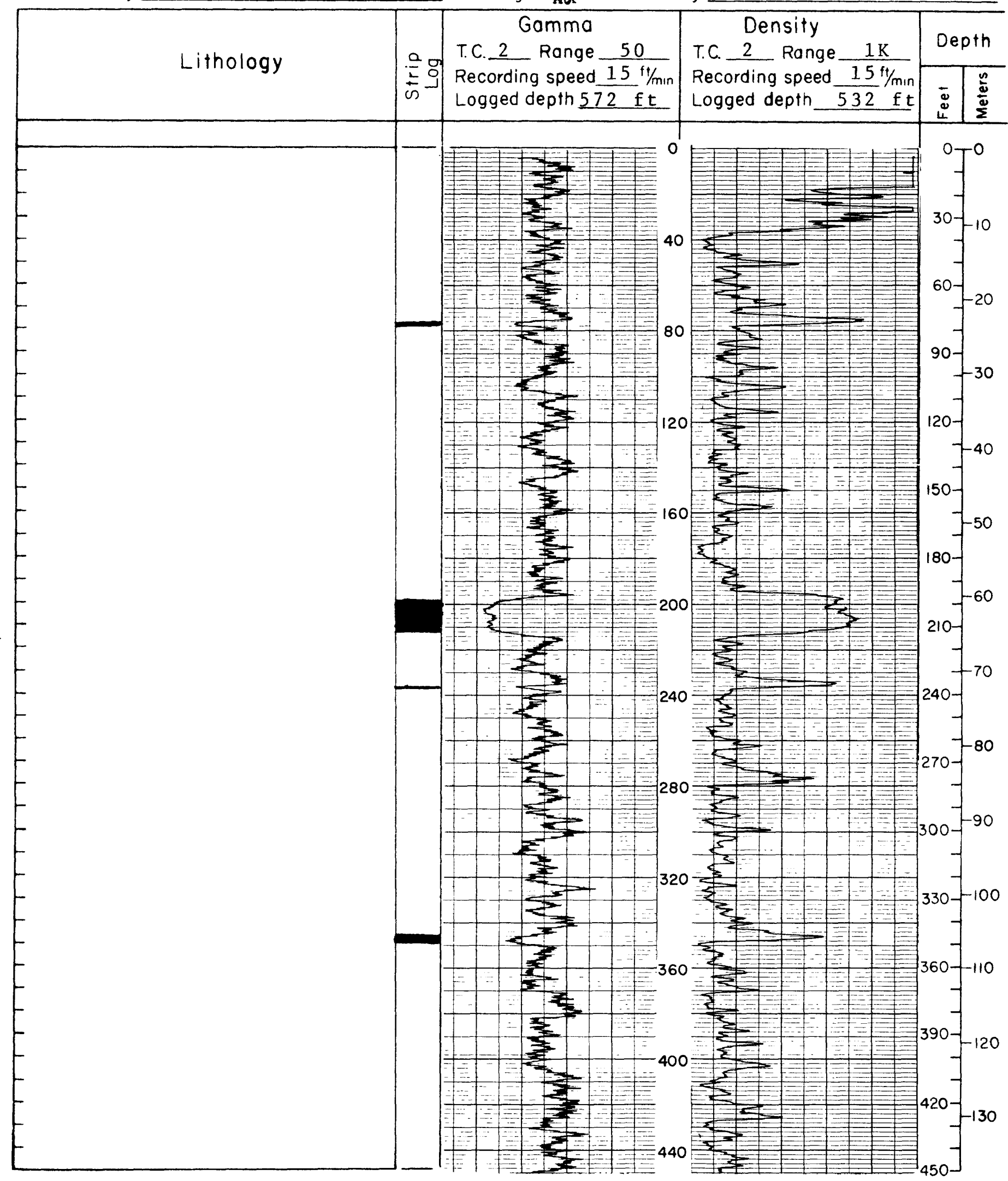


Hole number (continued)

US-77172

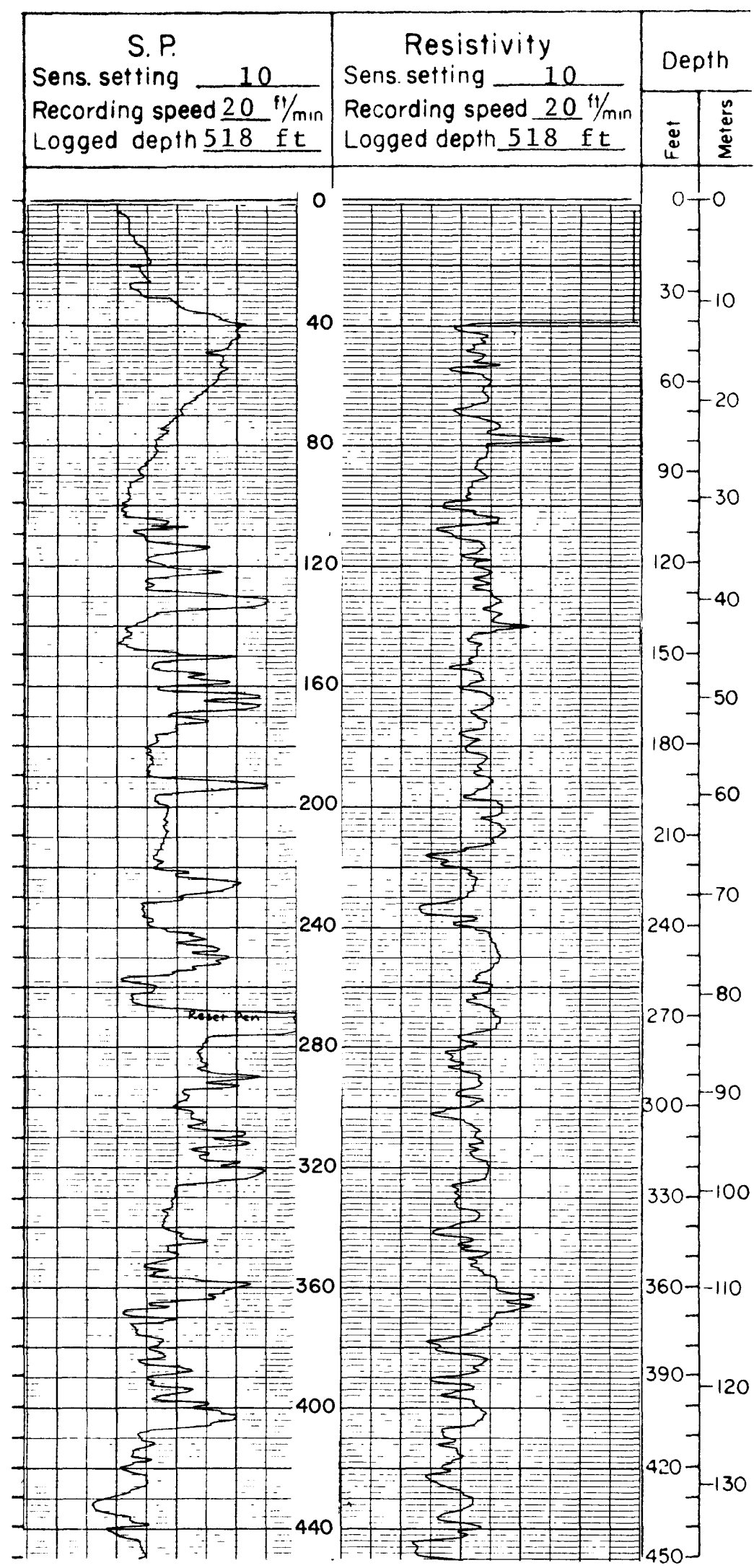


Hole number (continued)

$\underline{U S-77172}$

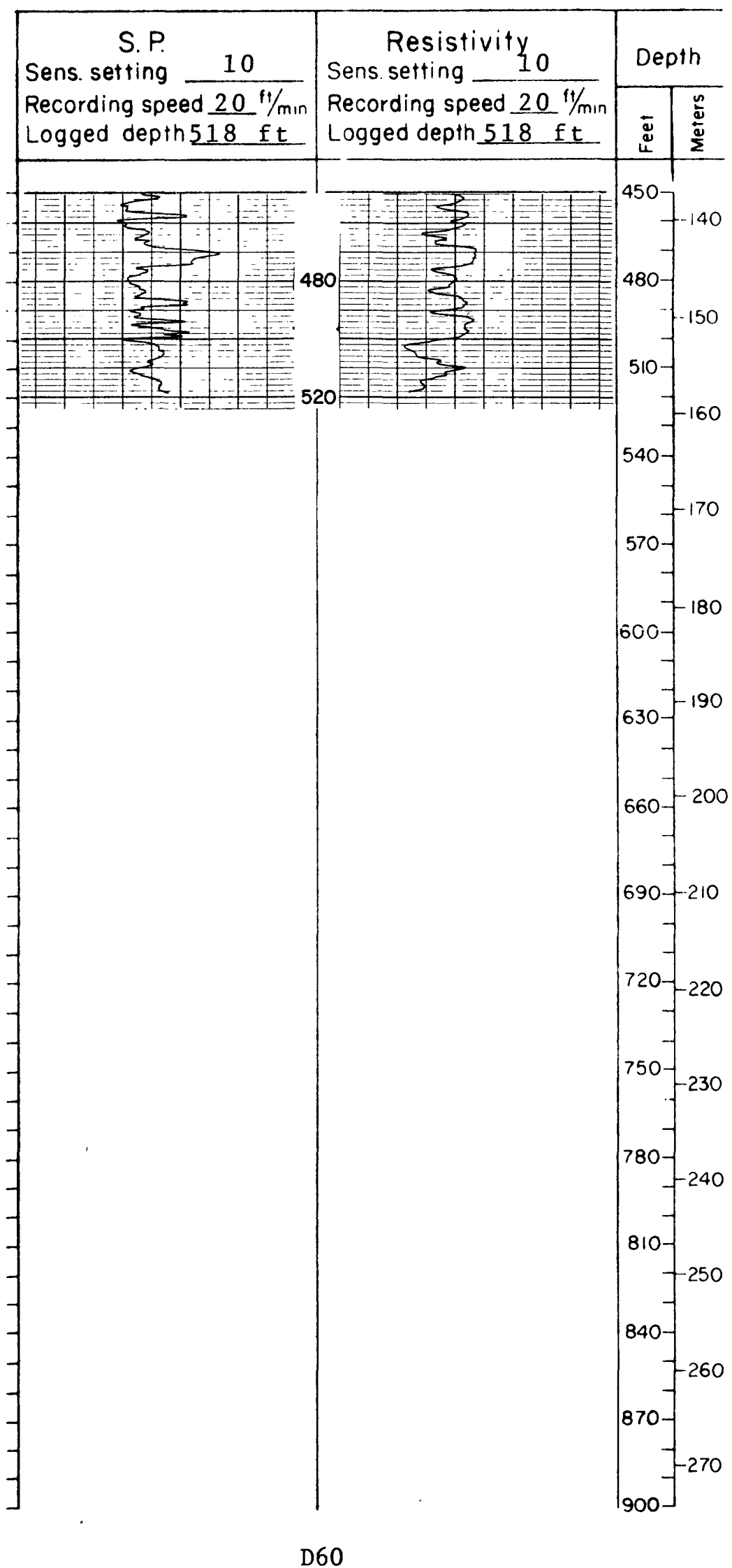


Hole no: US-77173 Map:BLM-NE 39 Dotelo/8/77 State Montana County: Dawson Elev. $2719 \mathrm{ft}$ Location:T. $17 \mathrm{~N}, \mathrm{R} .52 \mathrm{E}, \mathrm{Sec} .4$, Tract CCCC Drilled depth: $200 \mathrm{ft}$

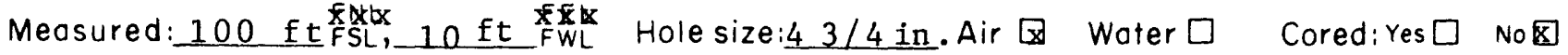

Remarks:

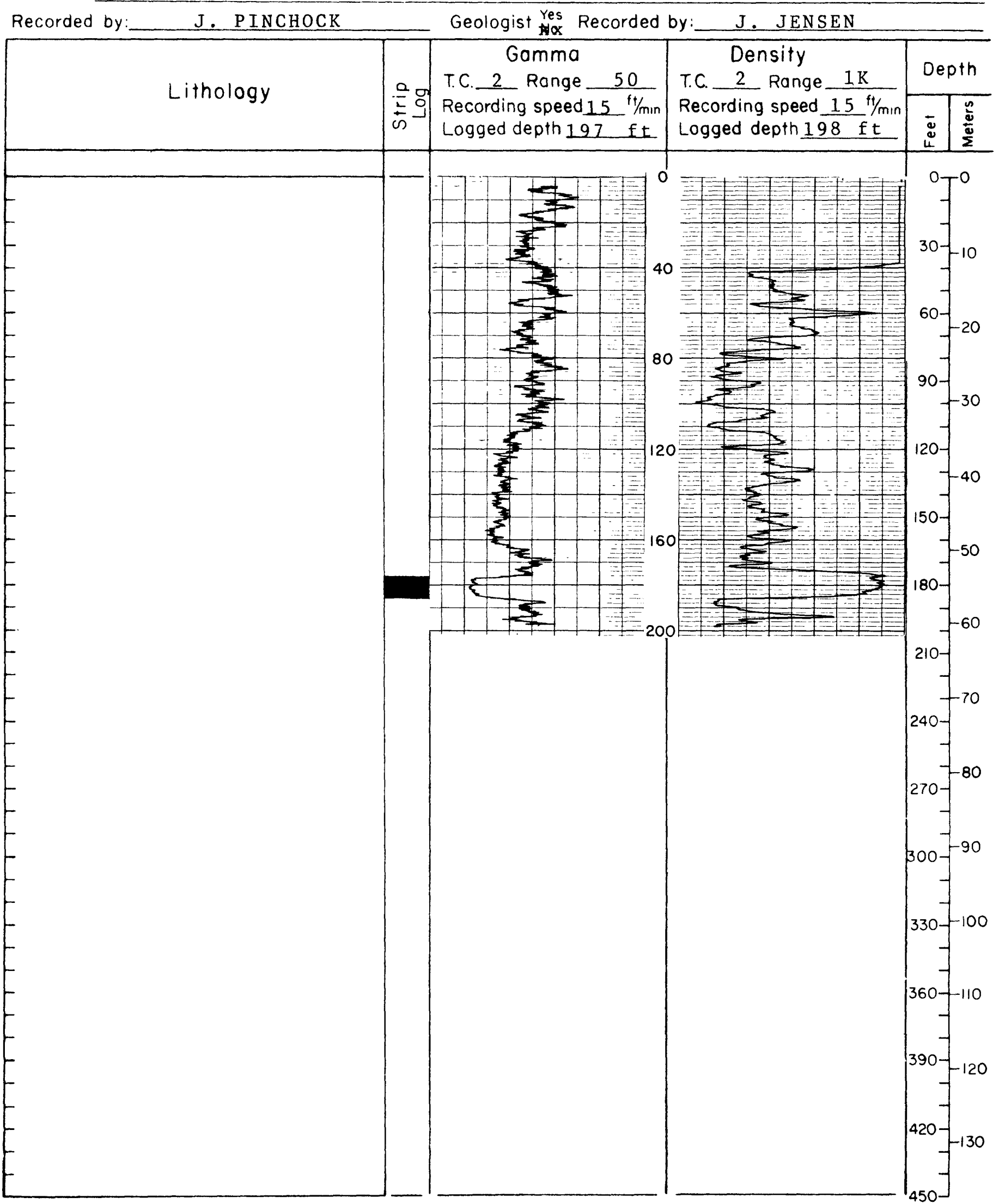


Hole number (continued)

$\underline{U S-77173}$

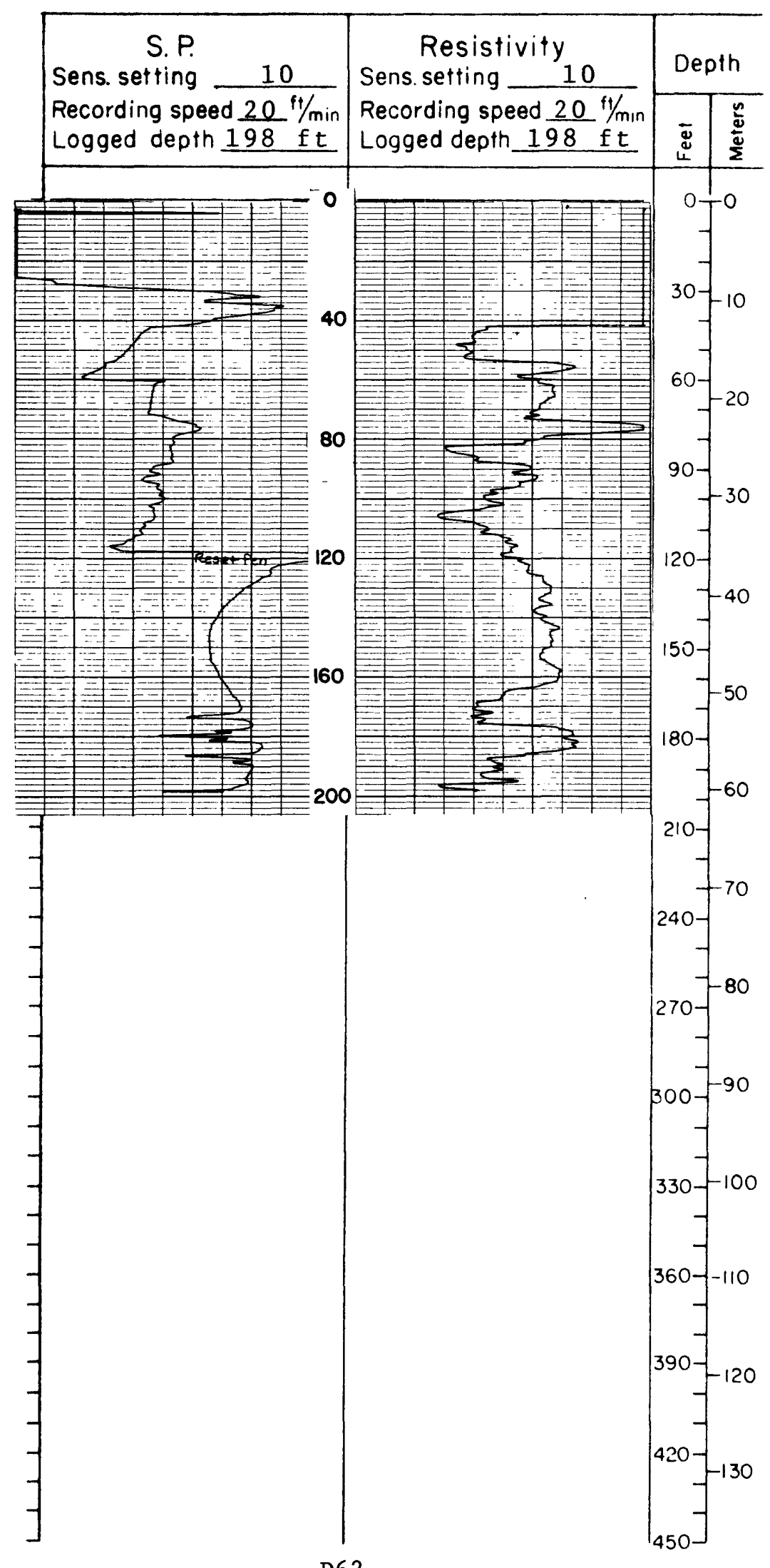




\section{Montana College of Mineral Science and Technology}

Hole no: US-77174 Map: BLM-NE30 Dote:10/9/77 StateMontana County:_McCone Elev. $2506 \mathrm{ft}$ Locotion:T. $22 \mathrm{~N}$. R. $49 \mathrm{E}$. Sec. 30 , Tract_BBBB Drilled depth: $140 \mathrm{ft}$ Measured: $35 \mathrm{ft}$ FN $100 \mathrm{ftFWL}$ Holesize $43 / 4$ in. Air $\otimes$ Water $\square$ CorediYes $\square$ No $\nabla$ Remarks:

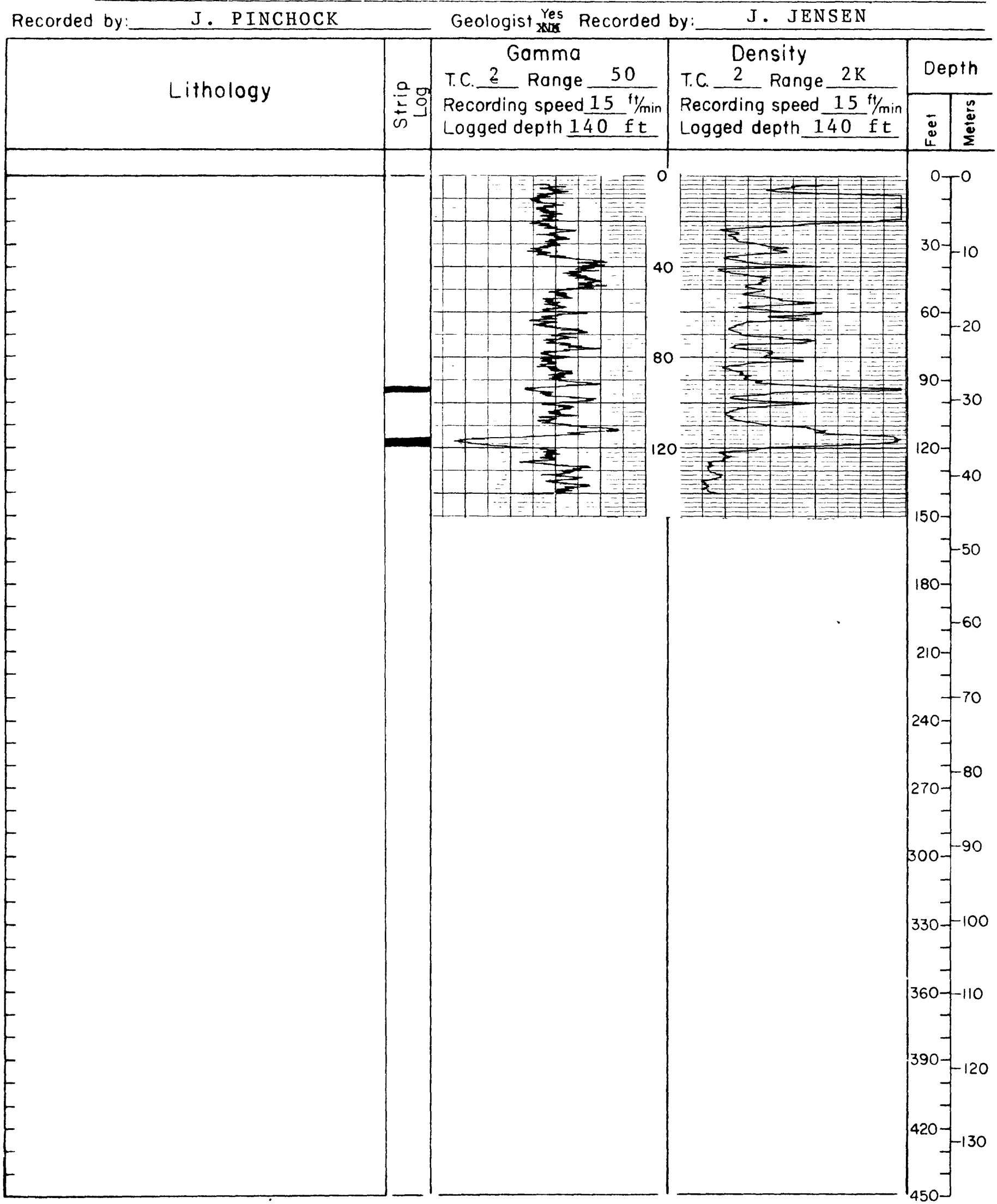


Hole noUS -77175 Map: BLM-NE 30 Dote $10 / 9 / 77$ Stotementana County: McCone Elev.:2498 ft Location: T. 22 N. R. 48 E. Sec. 20 , Troct BBBB Drilled depth: $160 \mathrm{ft}$

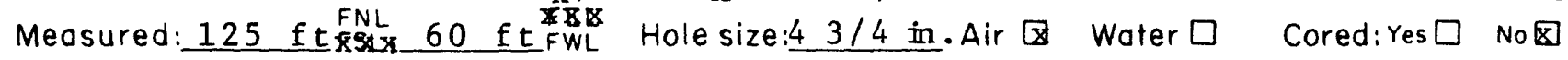
Remarks:

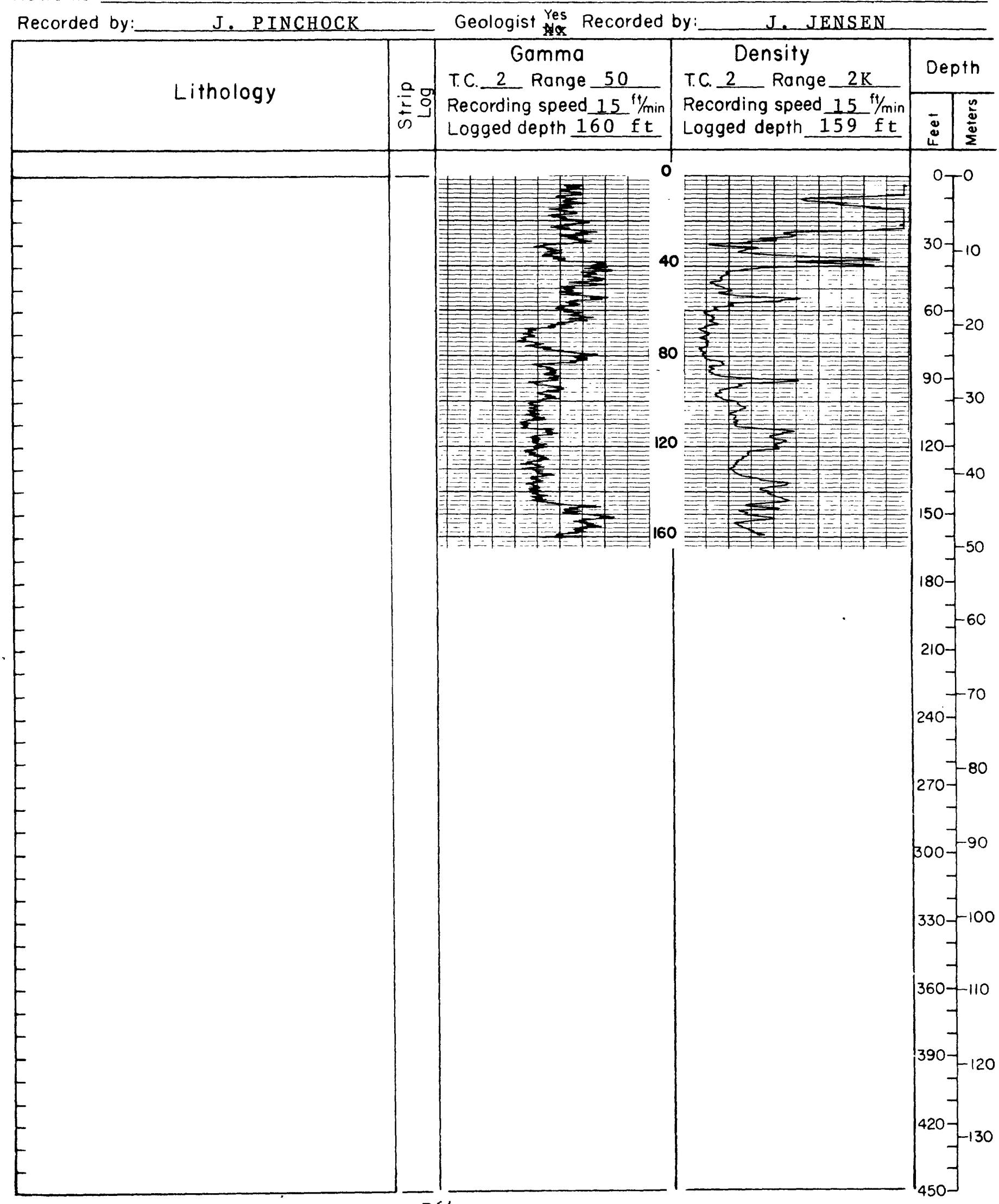


Montana College of Mineral Science and Technology

Hole no: US-77176 Map: BLM-NE30

Dote $\underline{10 / 9 / 77}$ State:Montana County: McCone Elev. $2467 \mathrm{ft}$ Location:T. $22 \mathrm{~N}, \mathrm{~S}, \mathrm{R} .4 \mathrm{E}$, Sec. 6 , Tract DAAD Drilled depth: $180 \mathrm{ft}$

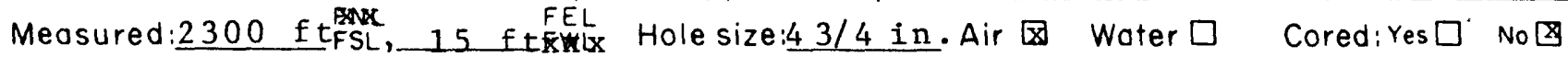
Remorks:

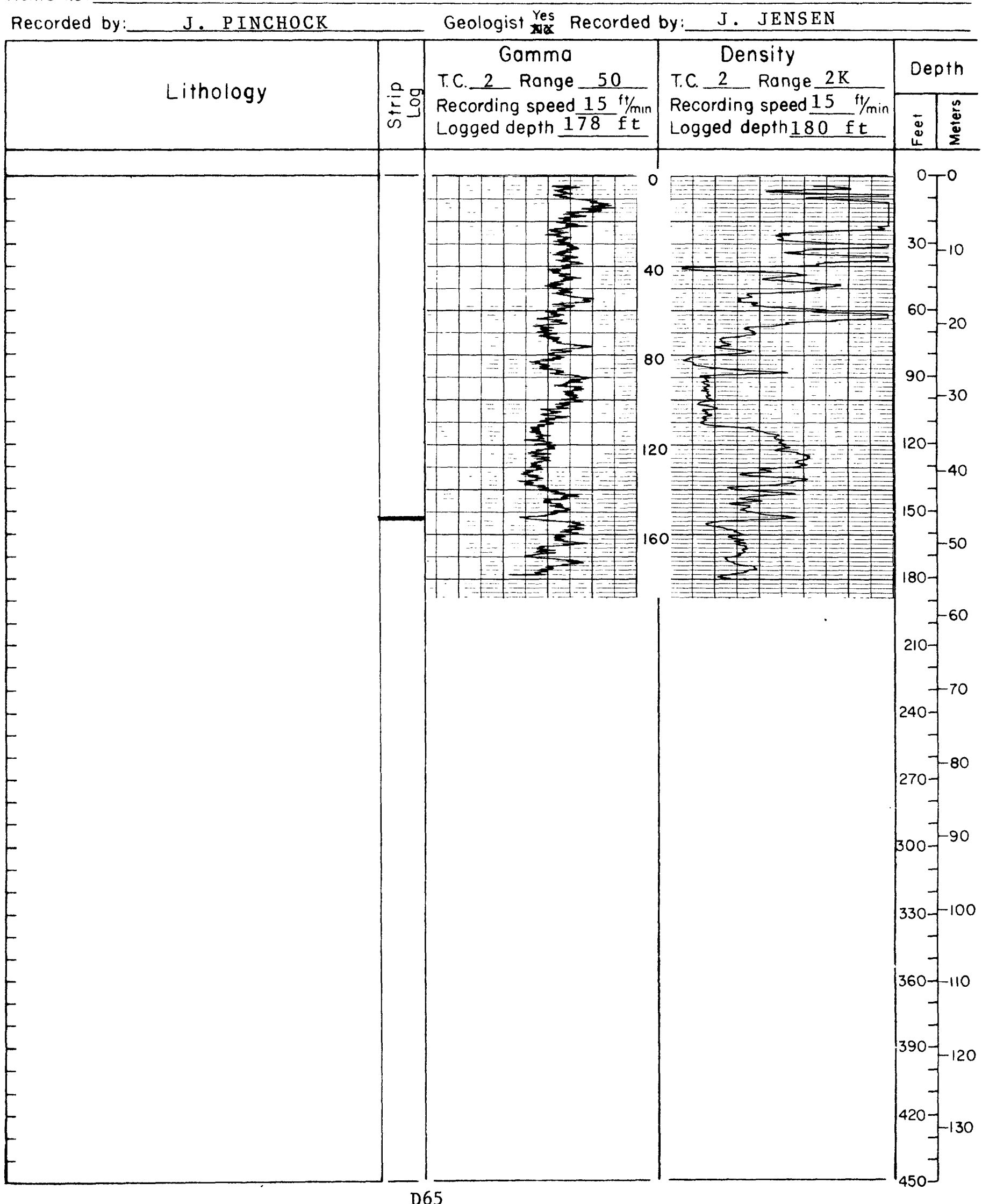


Hole no:US -77177 MOP: BLM-NE 30

Datelo/10/77 StateMont an a County:McCone

Elev. $2394 \mathrm{ft}$ Location: T. $22 \mathrm{~N} . \mathrm{K} .49 \mathrm{E}$. Sec. 4 , TractBBBB Drilled depth: $100 \mathrm{ft}$

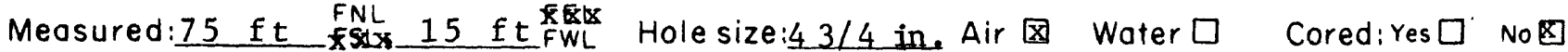
Remarks:

Recorded by:_ J. PINCHOCK Geologist Yes Recorded by: J. JENSEN

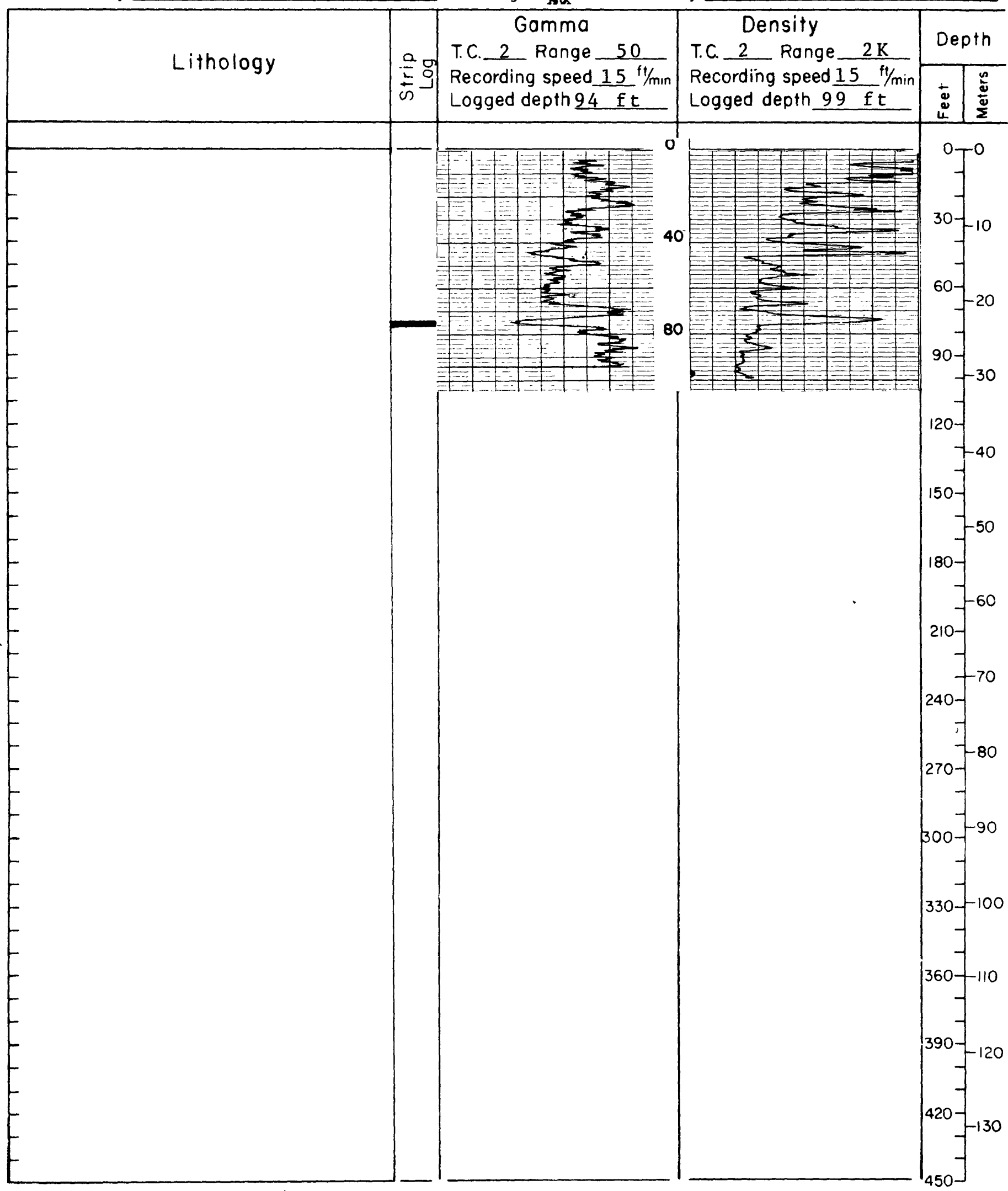


Montana College of Mineral Science and Technology

Hole no:US-Z7178 MOP:BLM-NE30

Date $10 / 10 / 77$ State:Montana County: McCone

Elev. $2311 \mathrm{ft}$ Locotion:T. $22_{\mathrm{N}}^{\mathrm{N}}, \mathrm{R} .49 \mathrm{E}$. Sec. 34 , Troct_CCCC Orilled depth: $200 \mathrm{ft}$

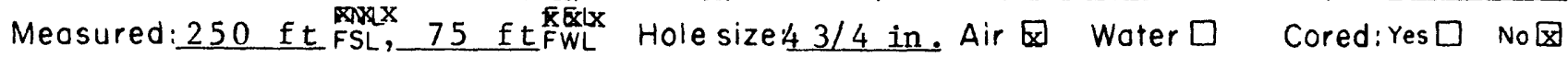
Remarks: Sand and gravel caved on pipes, no density $10 \mathrm{~g}$

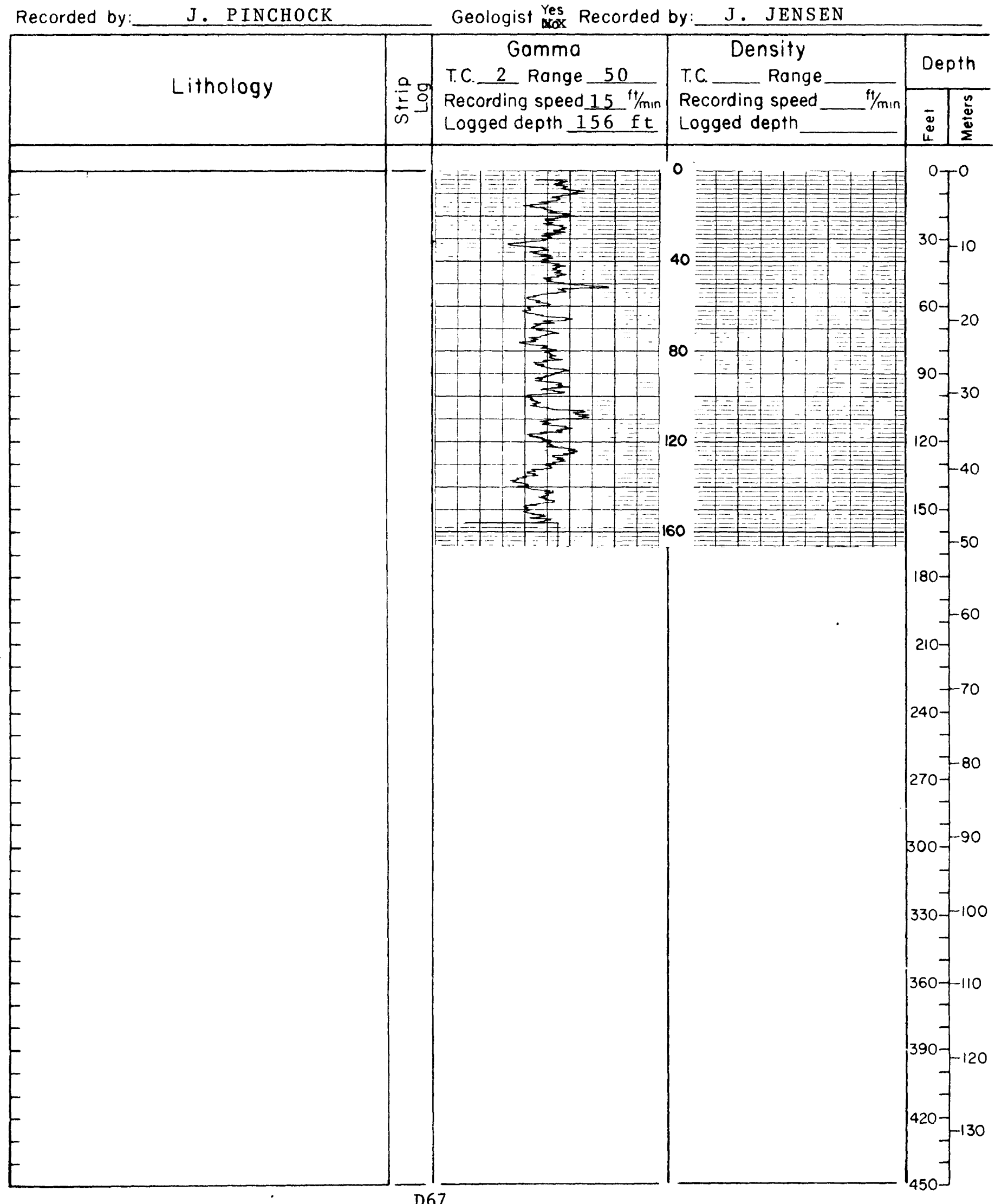


Hole no:US-77179 MOp:BLM-NE 31

Elev. $2491 \mathrm{ft}$ Location: T. 20 N. R. 50 E. Sec. 20 , Tract_B B B B

Dotelo $/ 17$ State:Montana County: Dawson

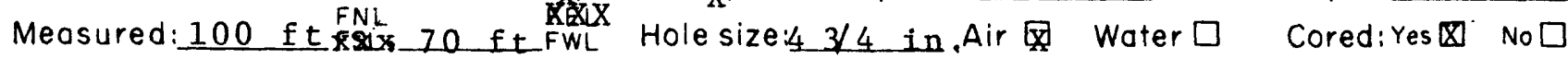
Remarks:

Recorded by: J. PINCHOCK

Lithology
Geologist Yes Recorded by

은멈

\section{Gamma}

T.C. 2 Range 50

Recording speed $15^{1 \%} / \mathrm{min}$ Logged depth 290 ft

\section{Density}

T.C. 2 Range $2 \mathrm{~K}$

Recording speed $15^{\mathrm{ft}} / \mathrm{min}$ Logged depth $294 \mathrm{ft}$

Depth

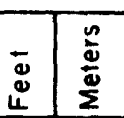

-
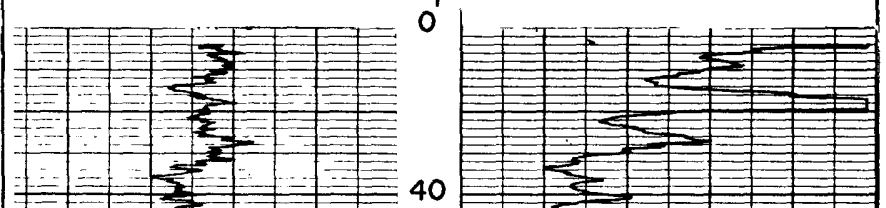

${ }^{0} T^{0}$
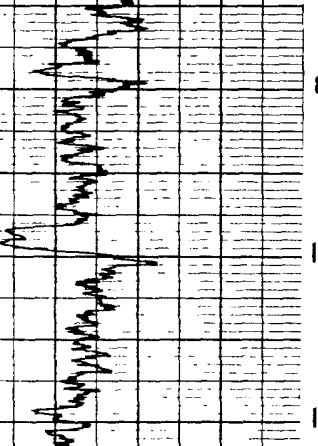

$\div-1$

$+$

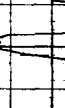

$\longrightarrow$
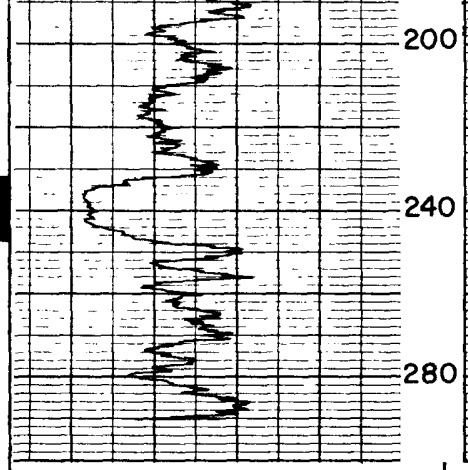

80
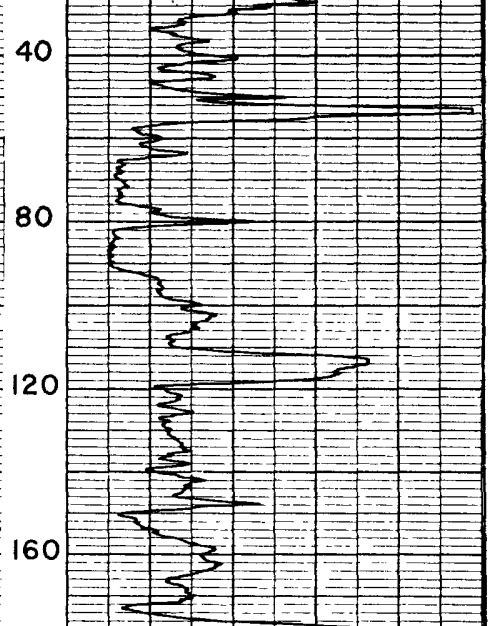

200
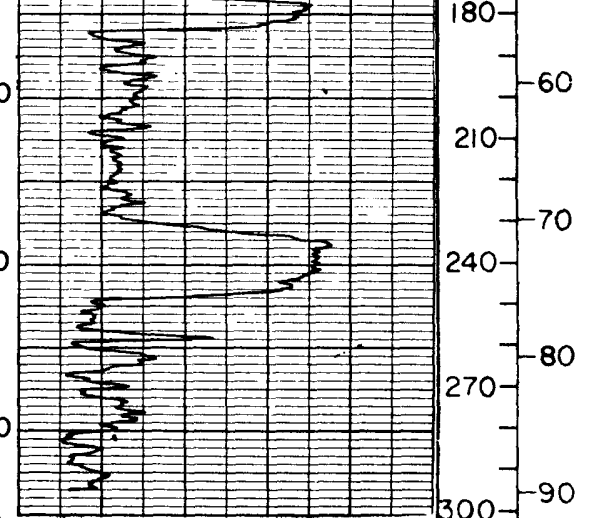
Hole number (continued)

$\underline{U S-77172}$

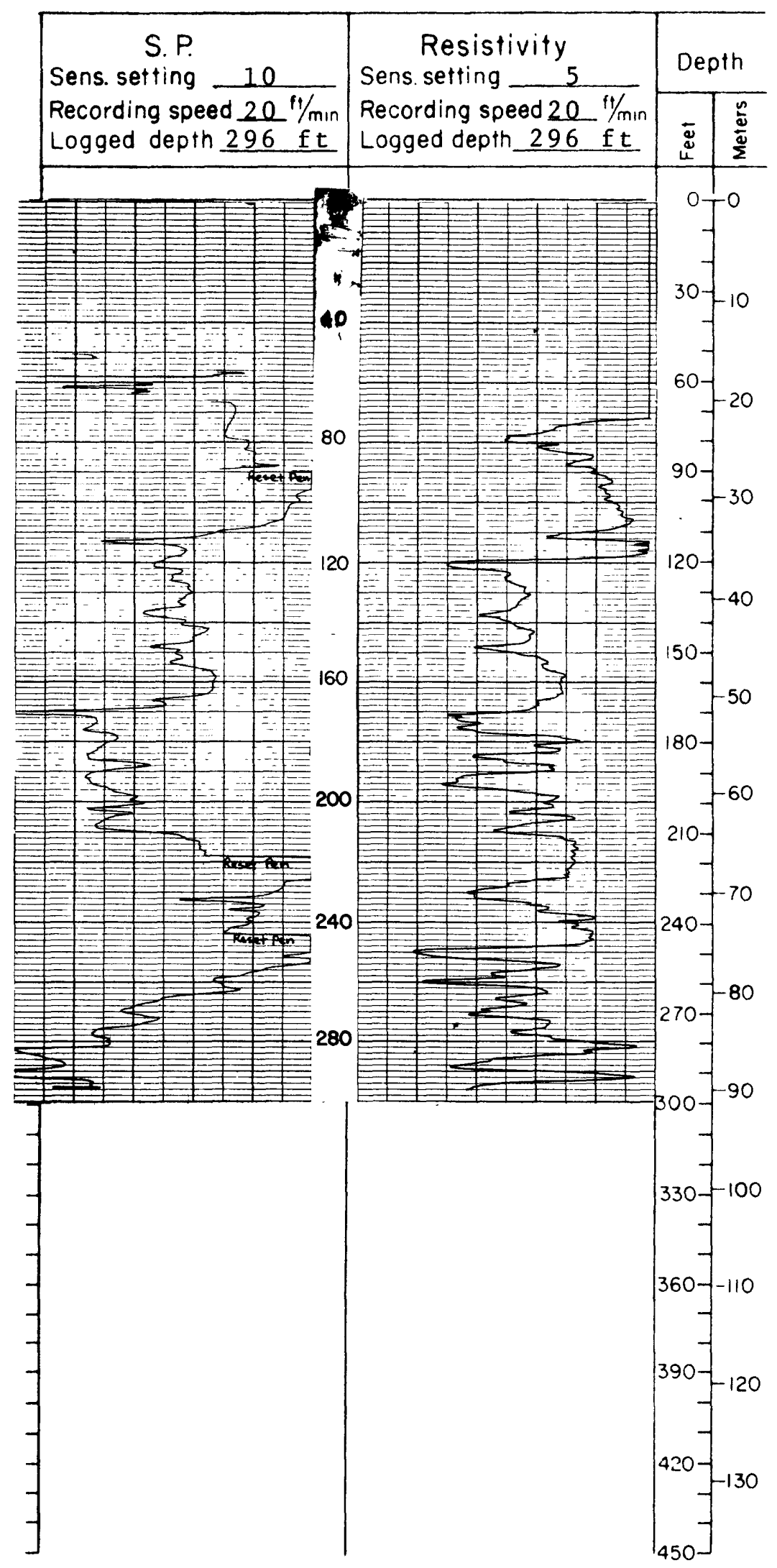




\section{Montana College of Mineral Science and Technology}

Hole no:US-77180 MOP: BLM-NE31

Datelo/117 StateMontana County: Dawson Elev. $2719 \mathrm{ft}$ Locotion: T.19 N. R. 50 虫 Sec. 10 , Tract DDDD Drilled depth:362 ft Meosured:50 ft FSL, $100 \mathrm{ftEWX}$ Holesize $43 / 4$ in. Air $₫$ Woter $\square$ Cored:Yes $\square$ No $\otimes$ Remarks:

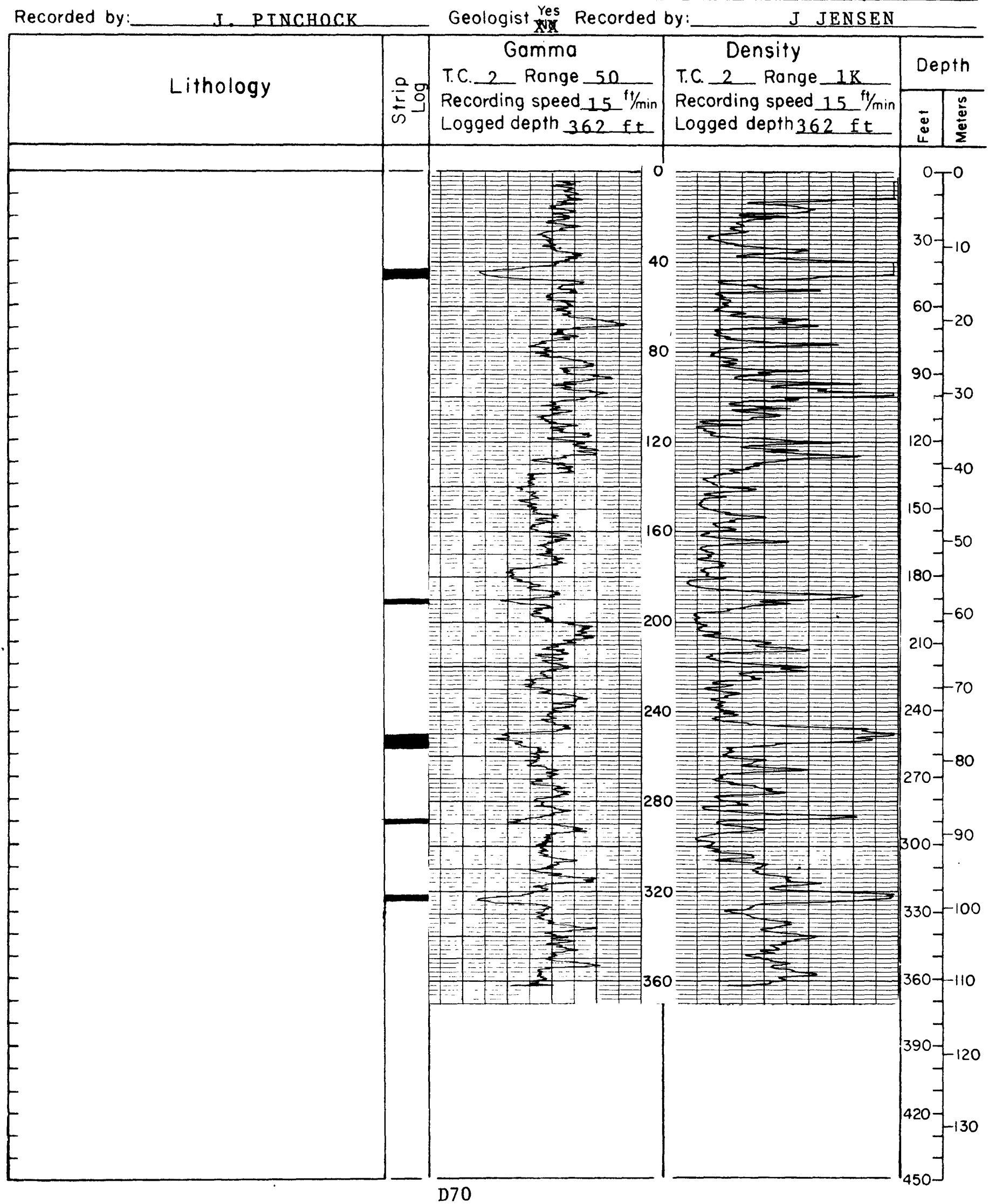


Montana College of Mineral Science and Technology

Hole noUS-77181 MOP:_BIM-NE31

DotelepllZ7StateMontana County: Dawson

Elev. $2649 \mathrm{ft}$ Location: T.21 N.R. 51 E. Sec. 30 , Tract BDAD Orilled depth:_ $360 \mathrm{ft}$

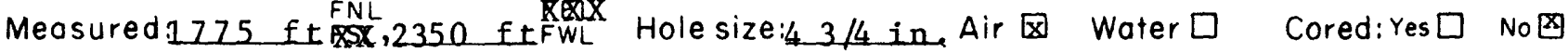

Remorks:

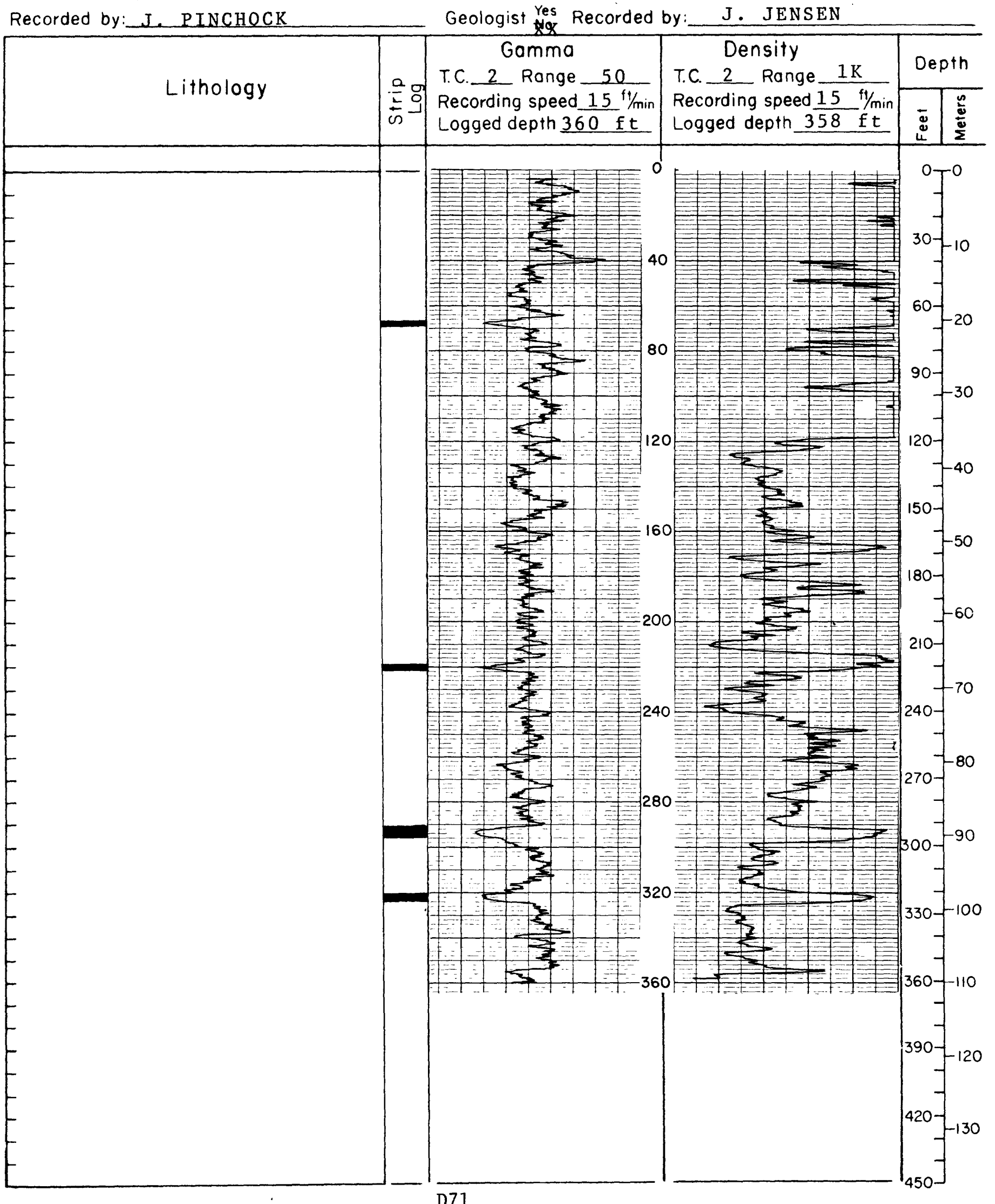


Hole no: IS -77182 Map:BLM-NE 31

Date10/2/77 State:Montana County: Dawson

Elev. $2491 \mathrm{ft}$ Locotion: T. $21 \mathrm{~N}, \mathrm{R} .51 \mathrm{E}, \mathrm{Sec} .18$, Tract CBCC Drilled depth: $280 \mathrm{ft}$ Measured: $1500 \mathrm{ft}$ FSL, $50 \mathrm{ft} F \mathrm{FL}$ Hole size:43/4 in. Air 0 Water $\square$ Cored:Yes $\square$ No Remarks:

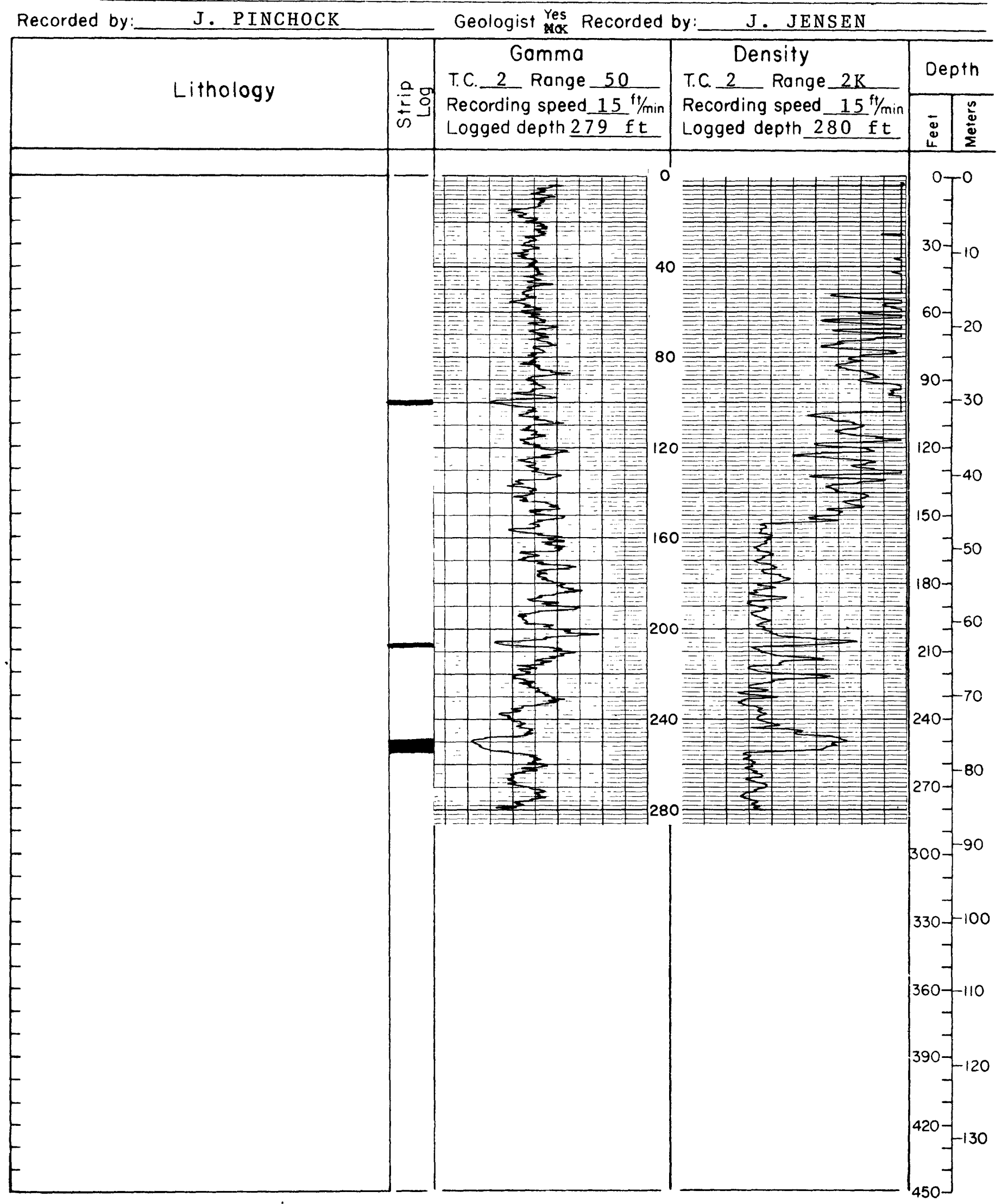


Hole number (continued)

$\underline{U S-77182}$

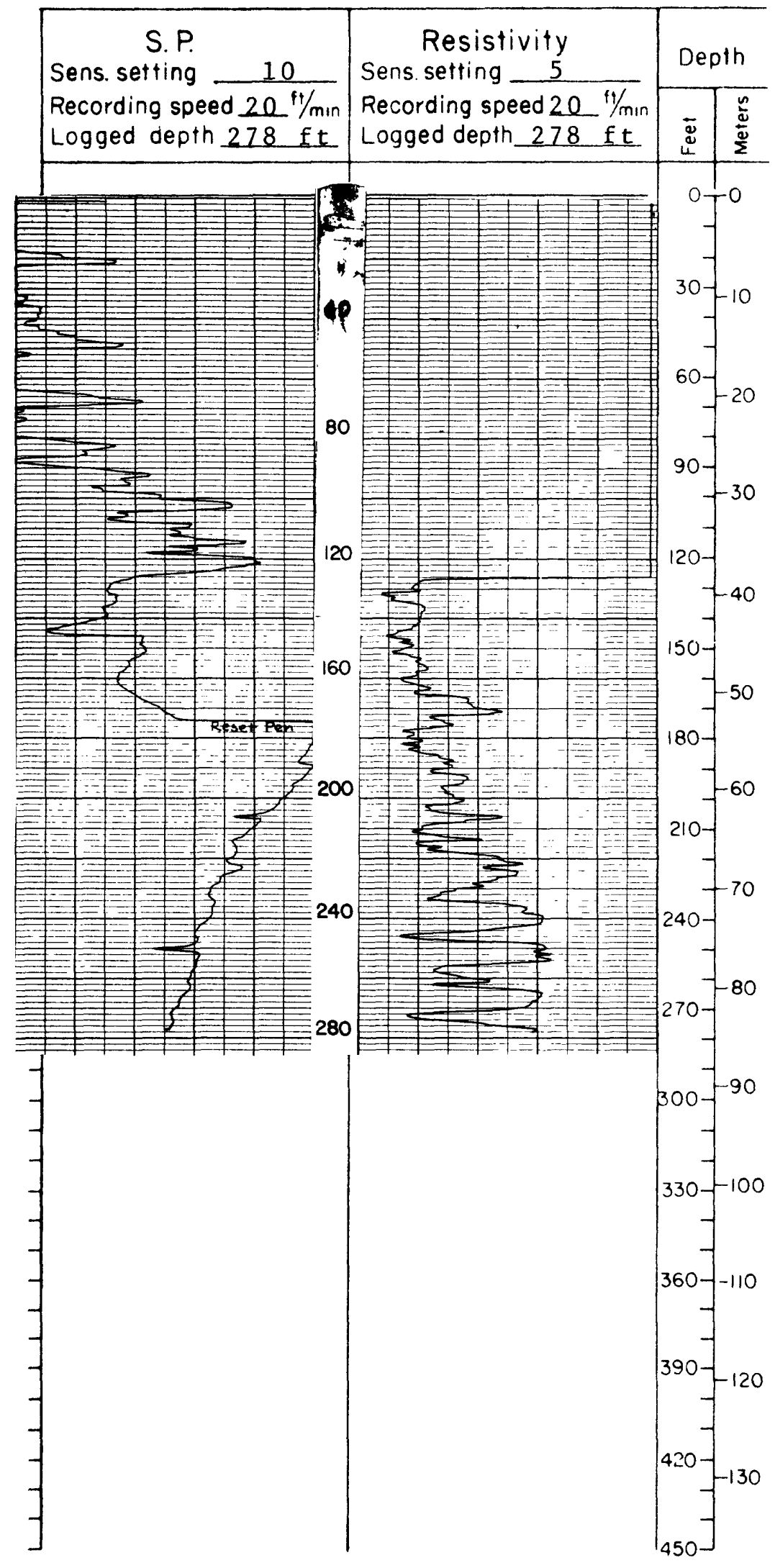


Hole no:US-77183 Mop: BLM-NE31

Dote10/11/775tate: Montana County: Dawson

Elev. $2541 \mathrm{ft}$ Location: T. 21 N. R. 50 E. Sec. 34 , Tract BBBB Drilled depth: $260 \mathrm{ft}$

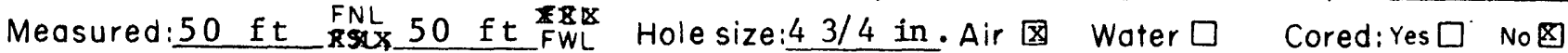

Remarks :

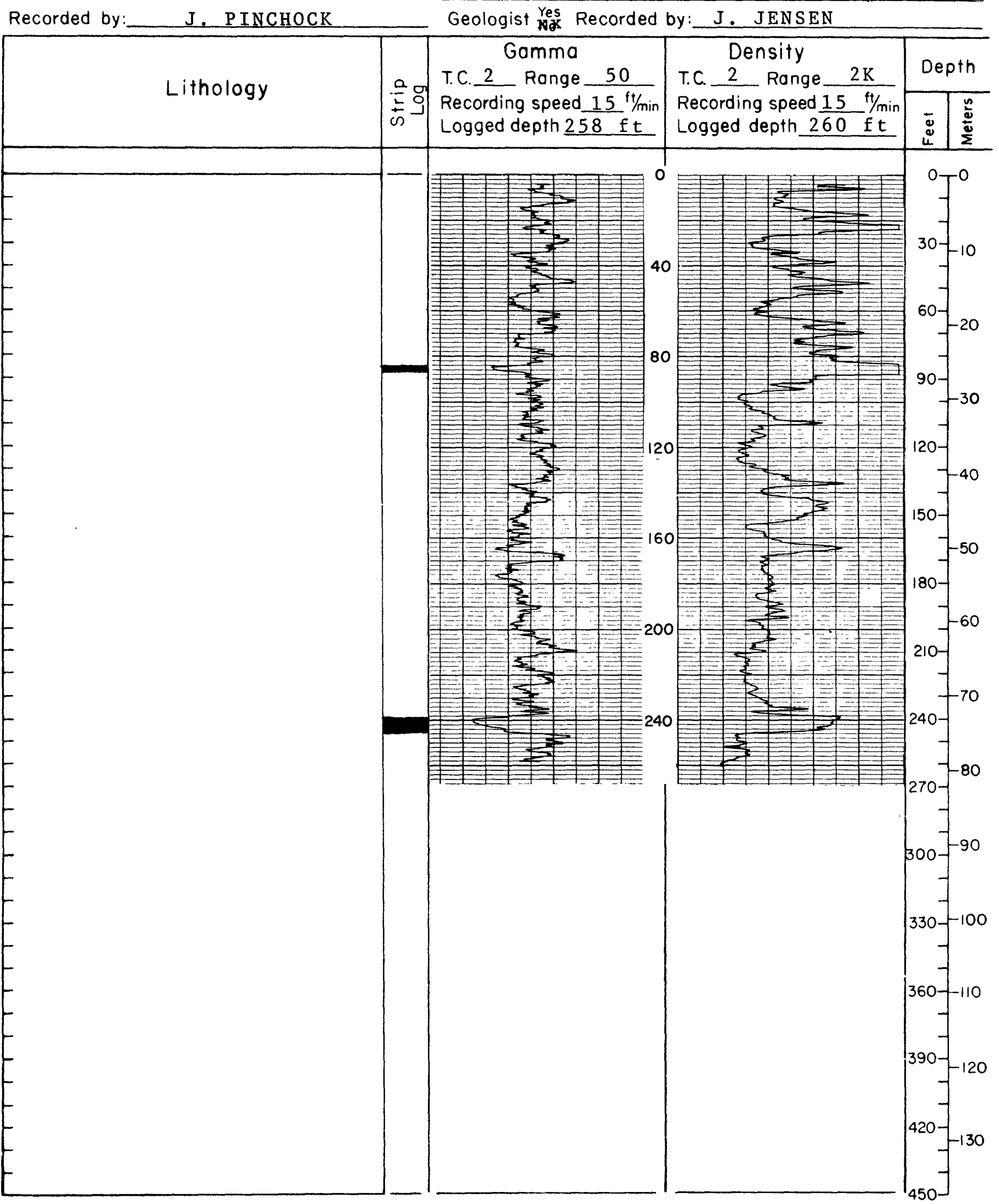


Hole no: IS -77184 MopLitt le Cha1k But teDote10/2477State:MontanaCounty: Garfie1d Elev. $2876 \mathrm{ft}$ Location: T.13 N. R. 44 E. Sec. 10 , Tract_CCCC Orilled depth: 300 ft

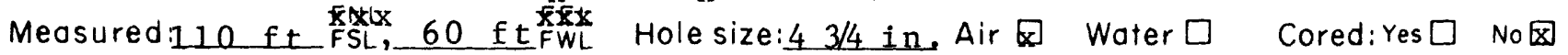
Remorks:

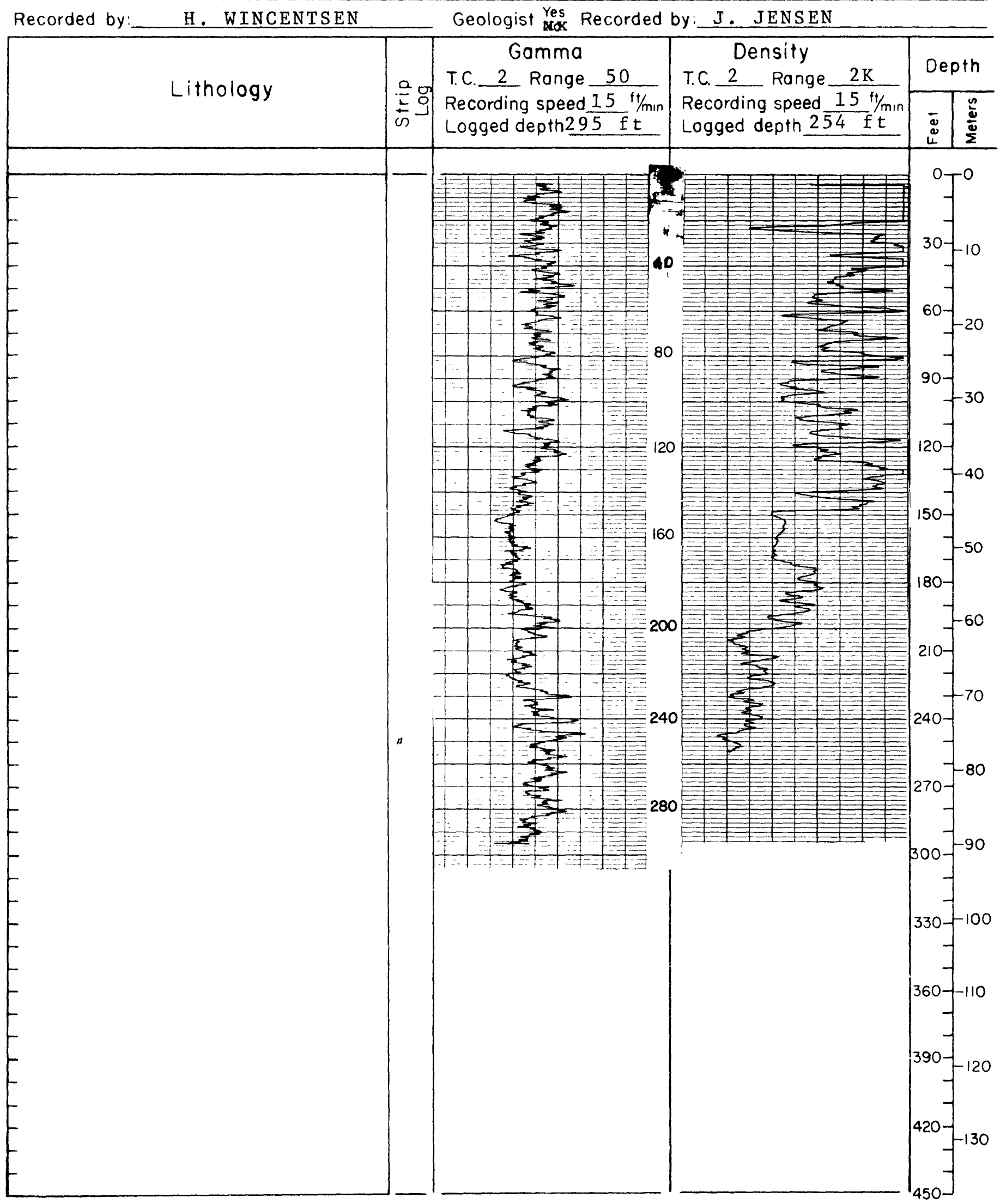




\section{Montana College of Mineral Science and Technology}

Hole no:US-77185 Map: Crow Rock

Dote10/19/7 7state:Montana County: Prairie

Elev. $2898 \mathrm{ft}$ Location: T. $13 \mathrm{~N}$, R. $45 \mathrm{E}, \mathrm{Wec} .15$, Tract_BCCB Drilled depth: $200 \mathrm{ft}$ Meosured:2200 ftF FL $96 \mathrm{ftFWL}$ Holesize:4 3/4 in. Air B Water $\square$ Cored:Yes $\square$ NoQ

Remorks:

Recorded by: HI. WINCENTSEN

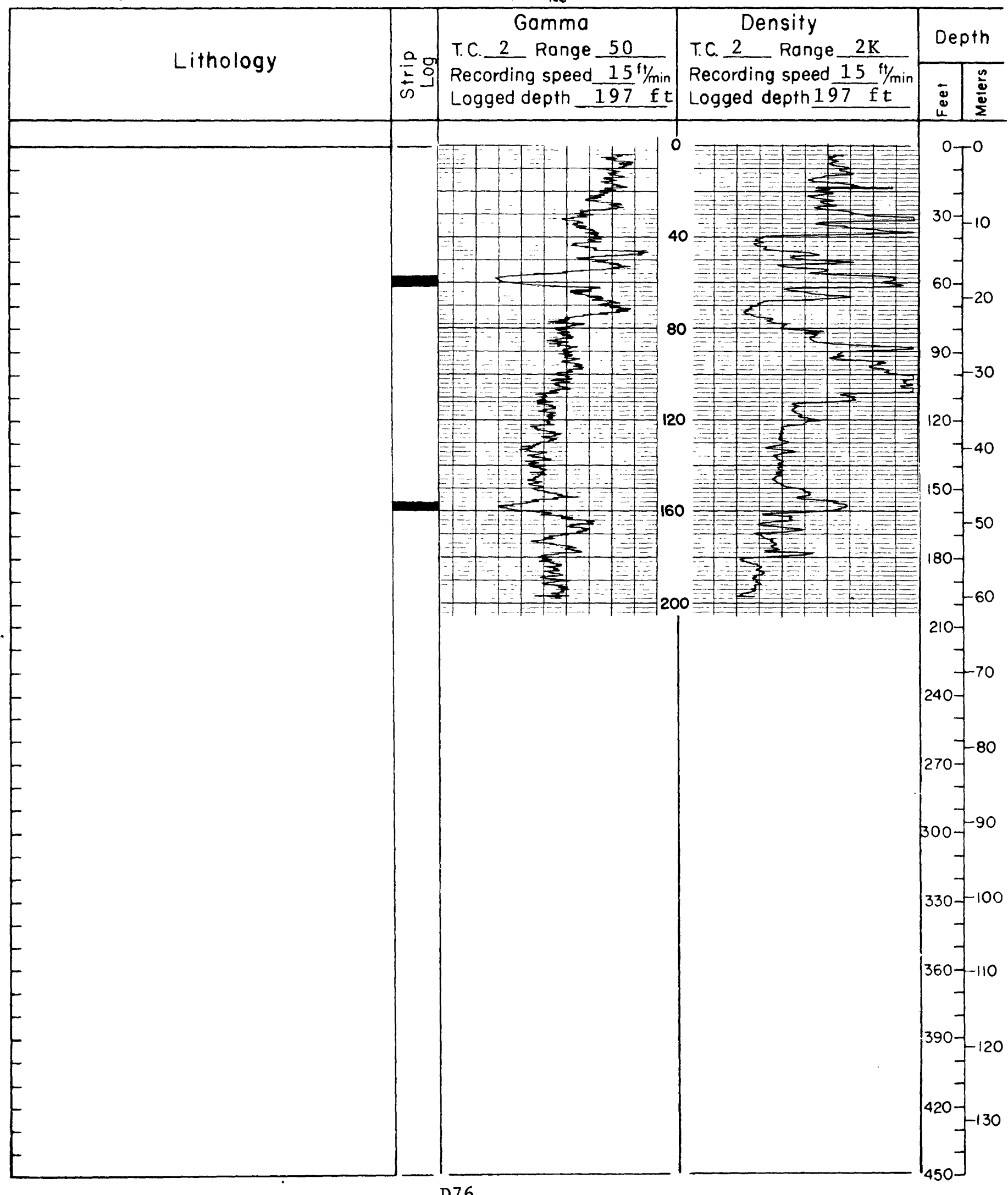


Montana College of Mineral Science and Technology

Hole no: US-77186 Mop: Crow Rock Date10/19/77StateMontana County: Prairie Elev. $2781 \mathrm{ft}$ Locotion:T. $14 \mathrm{~N}$. R. $45 \mathrm{~F}$. Sec. 34, Troct_CDCD Drilled depth: $300 \mathrm{ft}$ Meosured:200 ft FSL,1800 ft FWL Hole size: $43 / 4$ in . Air $\square$ Woter 8 Cored:Yes $\square$ No $\square$

Remarks:

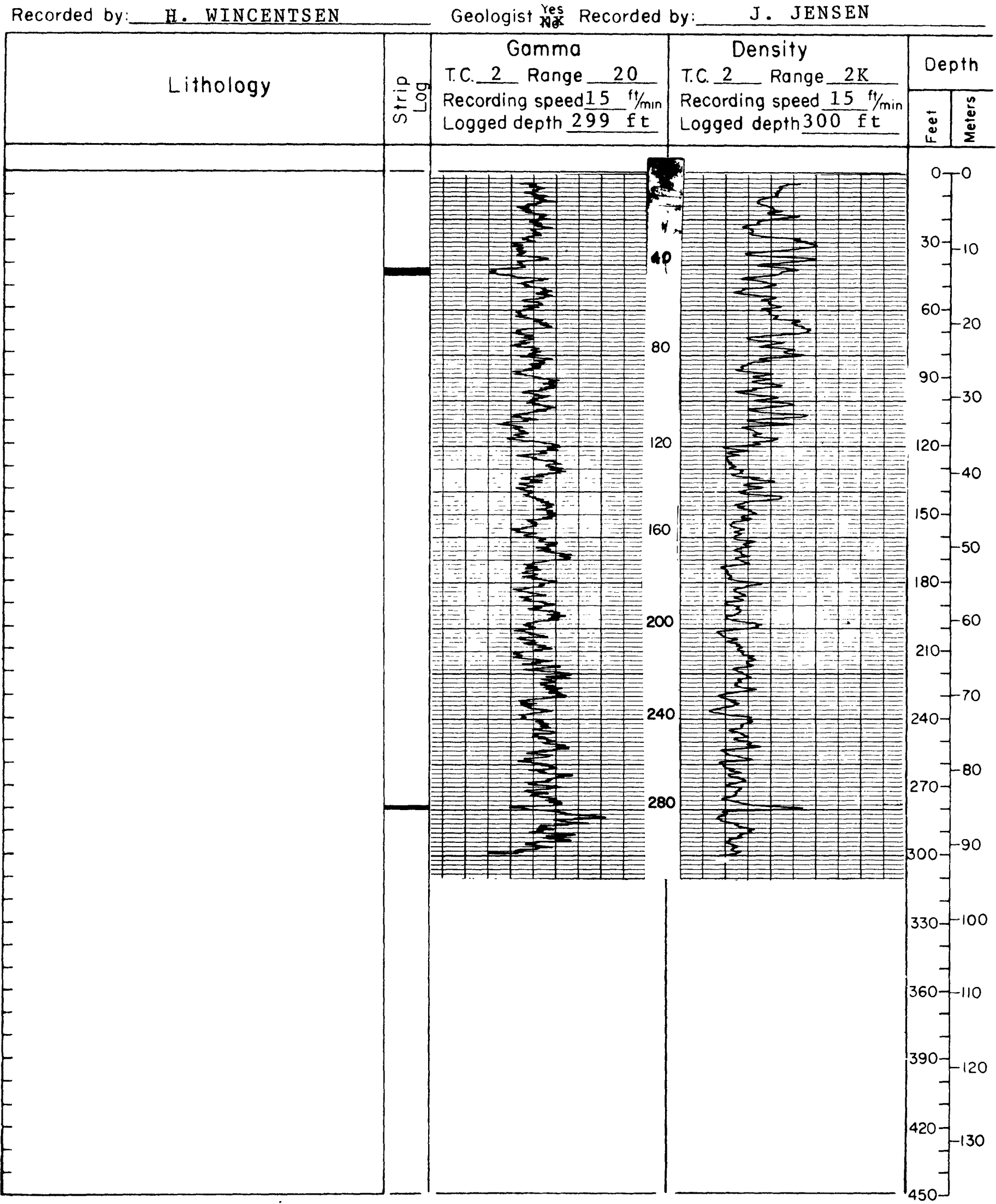


Hole number (continued)

US-77186

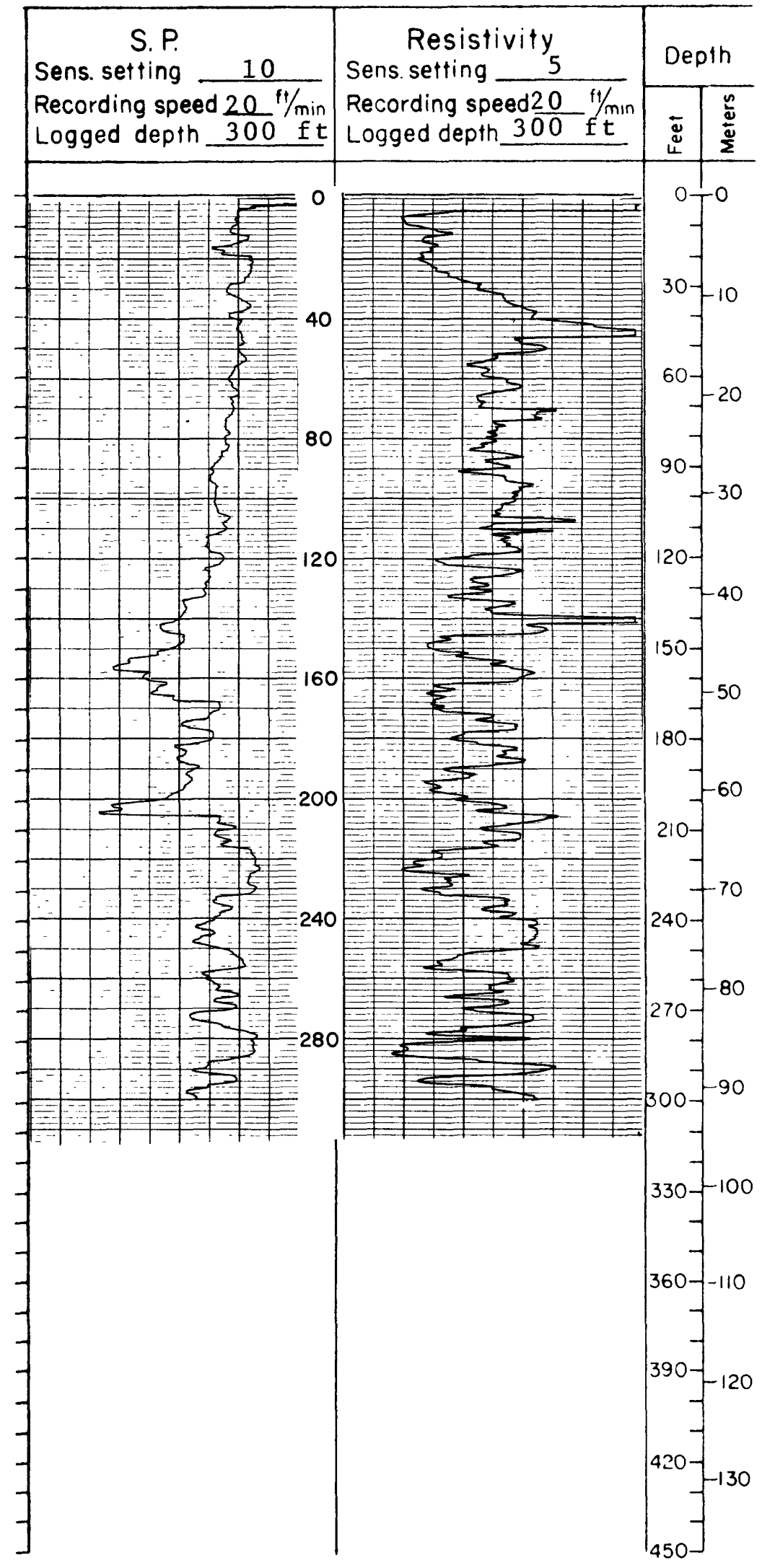




\section{Montana College of Mineral Science and Technology}

Hole no:US-77187 Map:Crow Rock

Date10/23/7 7State: MontanaCounty: Prairie

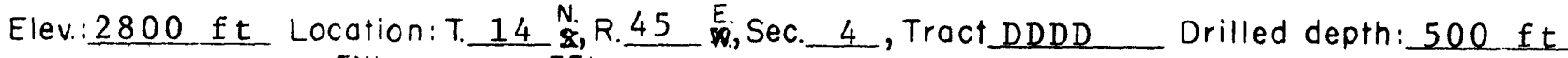

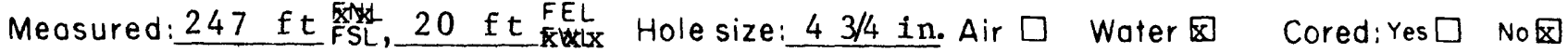
Remorks:

Recorded by: H. WINCENTSEN Geologist Yes Recorded by:___ J. JENSEN

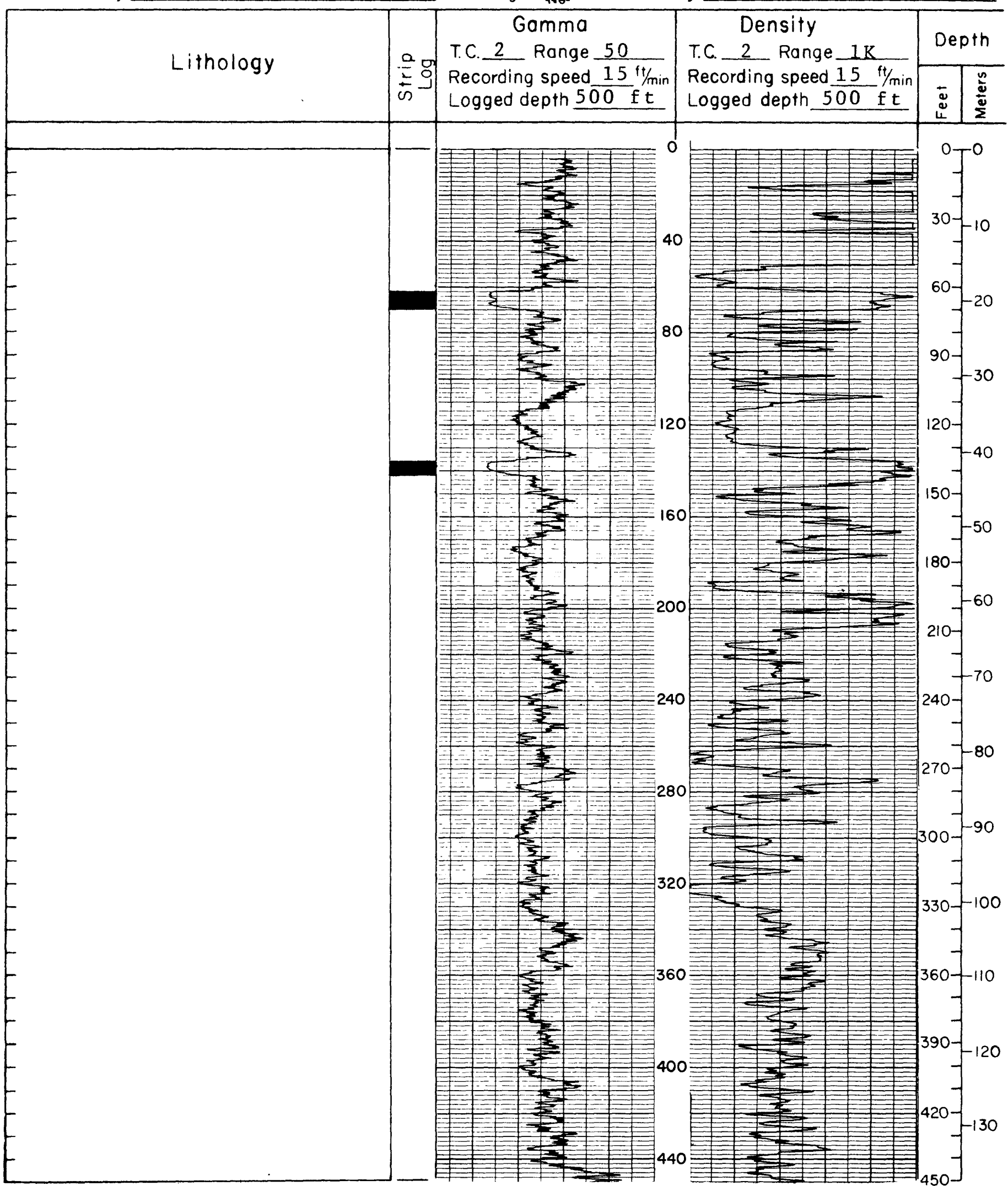


Hole number (continued)

US- 77187

Lithology

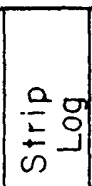

Gamma 2

\begin{tabular}{l|l} 
Recording speed $.15 \mathrm{ft} / \mathrm{min}$ & T.C. $\frac{2}{2}$ Range $\frac{1 \mathrm{~K}}{15 \mathrm{ft}}$ Depth
\end{tabular}

Logged depth $500 \mathrm{ft}$ Logged depth $500 \mathrm{ft}$

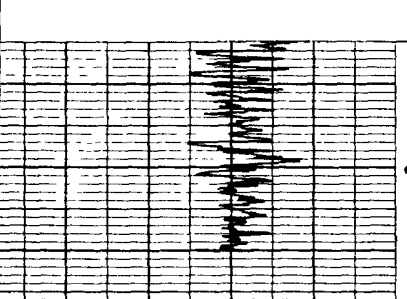

111

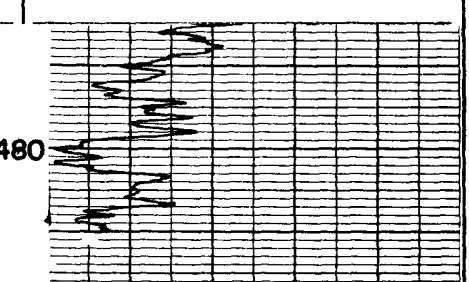

$+140$

480

$-150$

510

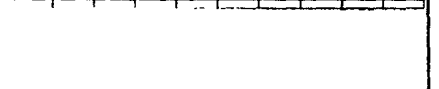

$510-$

$-160$

$540-$

$-170$

570

$600-180$

$630-190$

$660-200$

$690-210$

$720-220$

$750-230$

780

$-240$

810

${ }^{840-}-250$

870

$f_{900}-270$ 
Hole number (continued)

$\underline{U S-77187}$

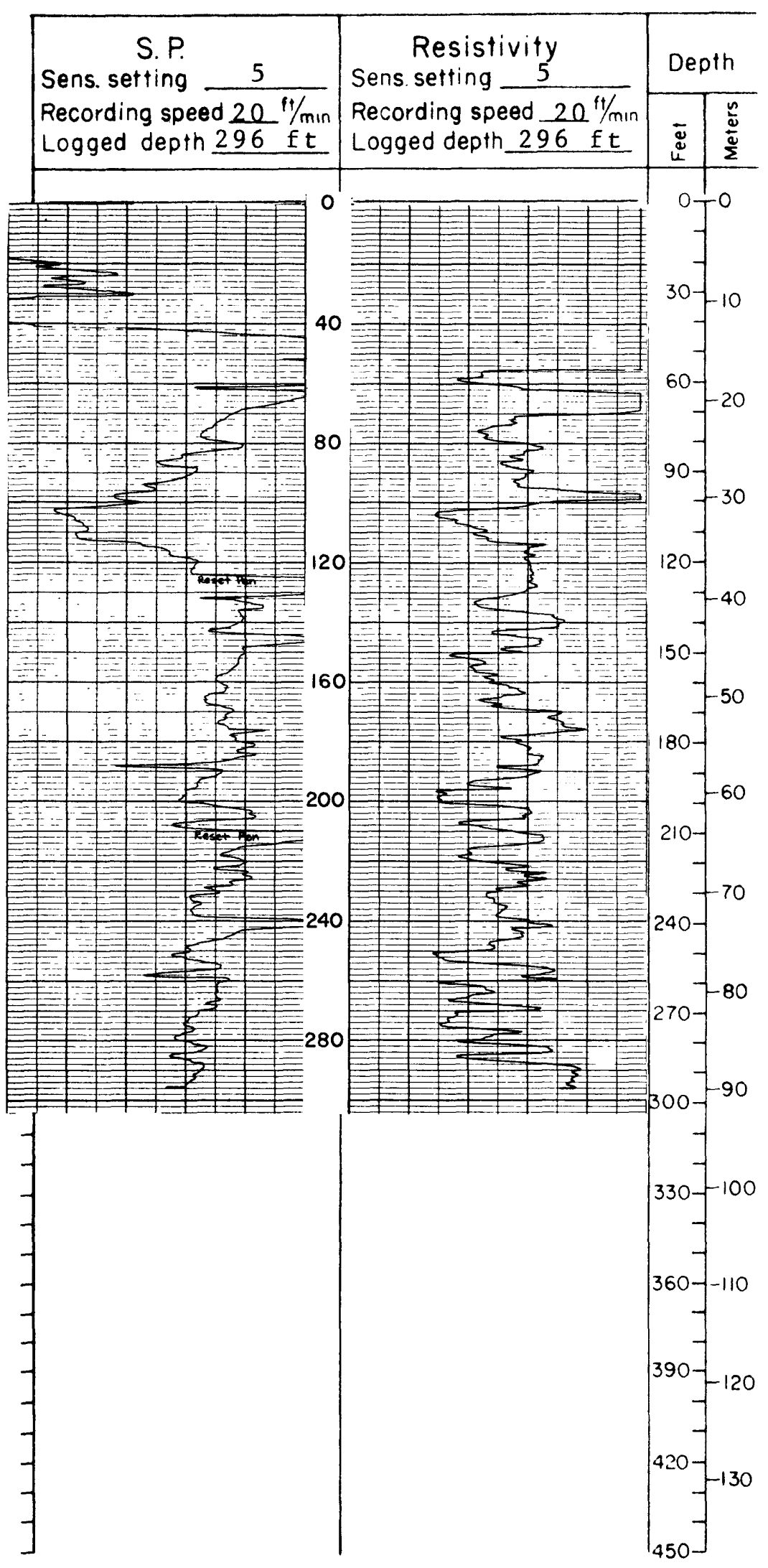




\section{Montana College of Mineral Science and Technology}

Hole no:US 77188 Map:Hedstrom Lake SE Dote 10/24/7 State:Montana County: Prairie

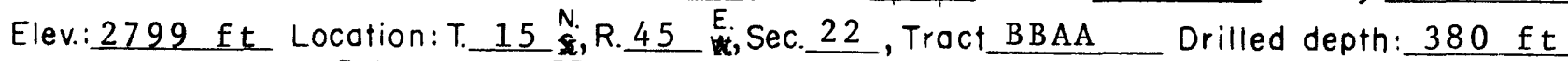

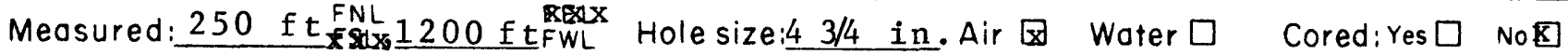

Remarks :

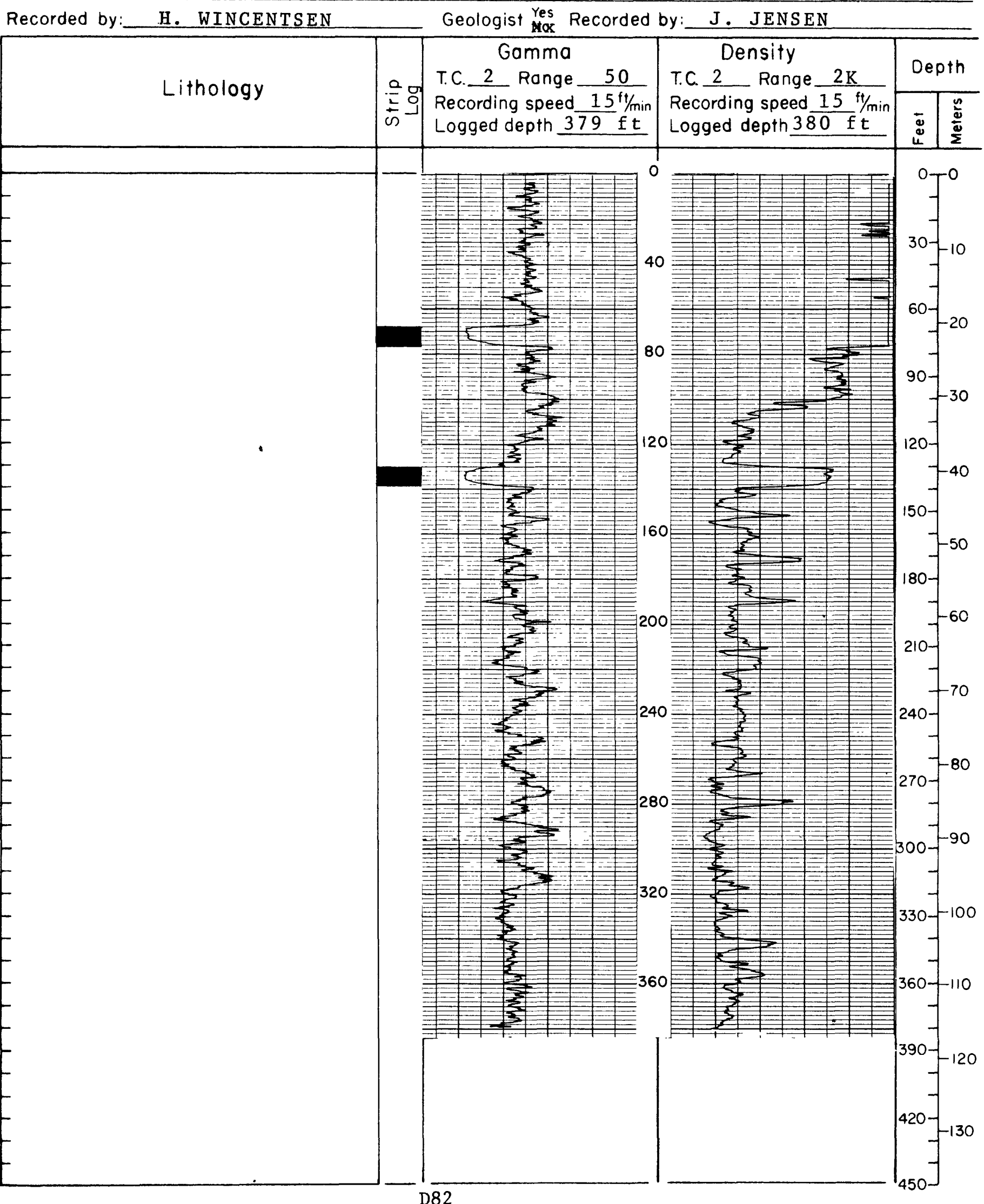


Hole no:US-77189 MapHedstrom Lake SE Dote:10/24/7 7tote:Montana County: Garfie1d Elev.: $2750 \mathrm{ft}$ Location: T. $15 \mathrm{~N}, \mathrm{~N} .44 \mathrm{E}, \mathrm{Hec} .26$, Tract_DADD Drilled depth: $300 \mathrm{ft}$

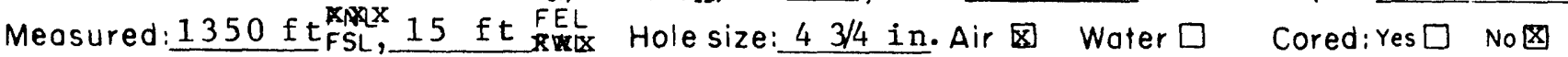
Remarks:

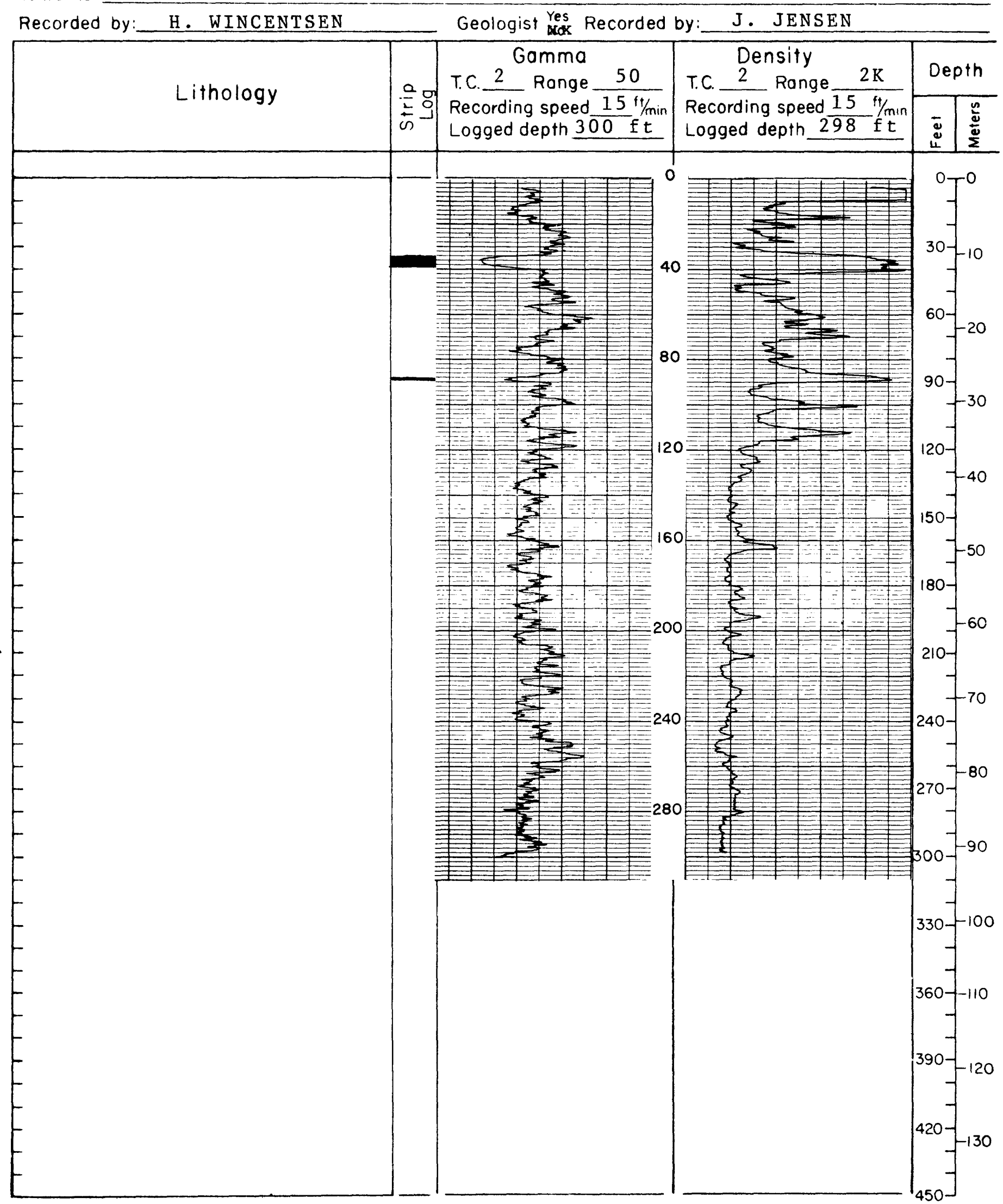




\section{Montana College of Mineral Science and Technology}

Hole no: US -77190 Mop: Crow Rock

Dote:10/2477State:Montana County: Garfie1d

Elev. $2720 \mathrm{ft}$ Locotion: T. $14 \mathrm{~N}, \mathrm{~S}, 44 \mathrm{E}, \mathrm{Kec} .24$, Tract_CCCC Drilled depth: $260 \mathrm{ft}$

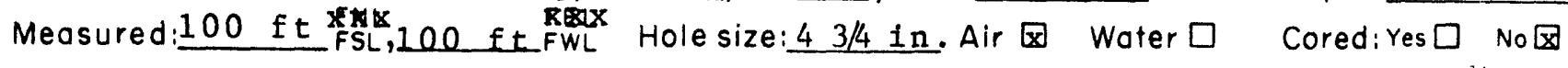
Remarks:

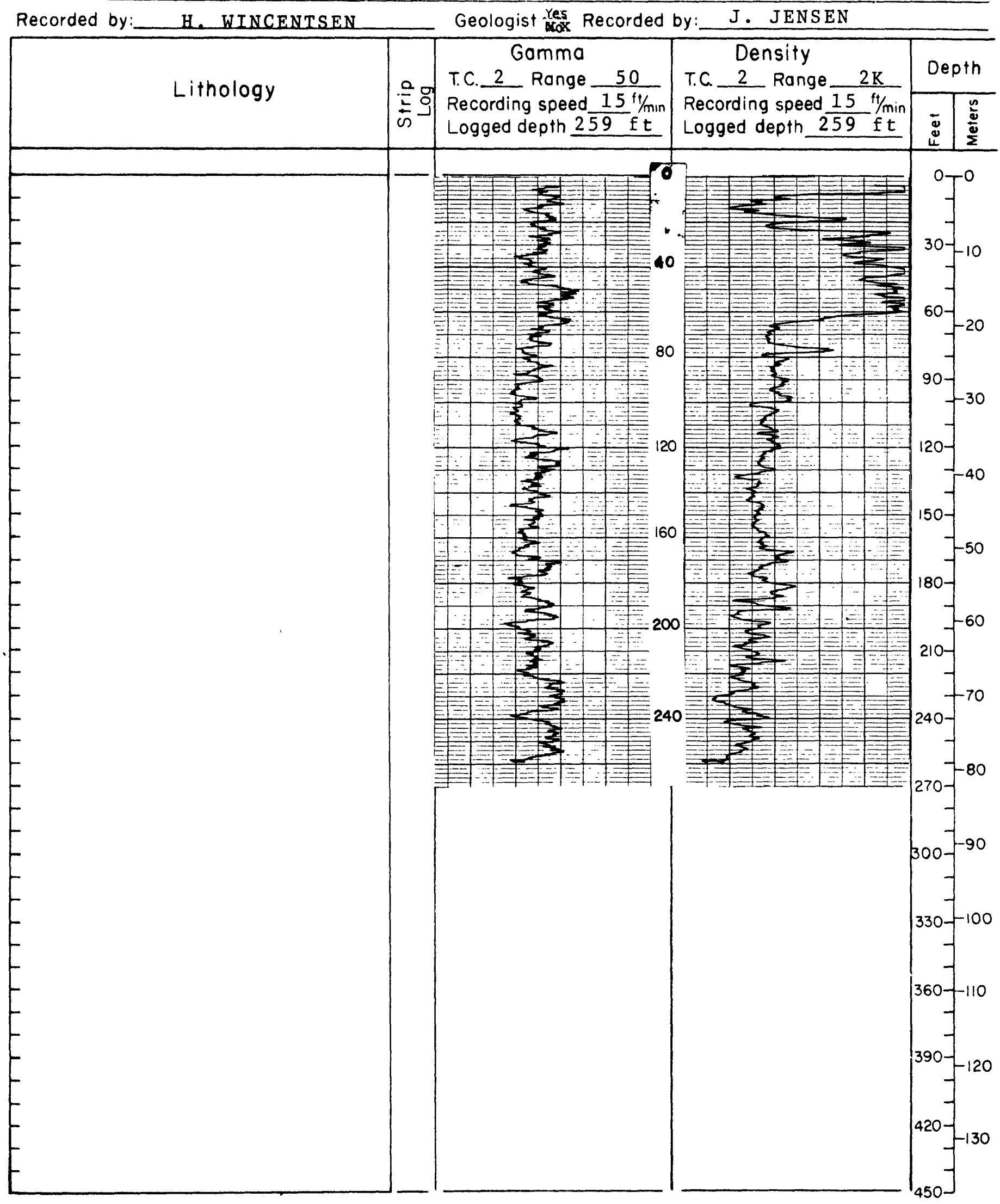


Montana College of Mineral Science and Technology

Hole no:US-77191 Map:Little Cha1k ButteDate1025/77 State:Montana County: Garfield Elev. $2815 \mathrm{ft}$ Location:T. 14 N. R. 44 E. Sec. 18 , Tract CADC Drilled depth: $370 \mathrm{ft}$

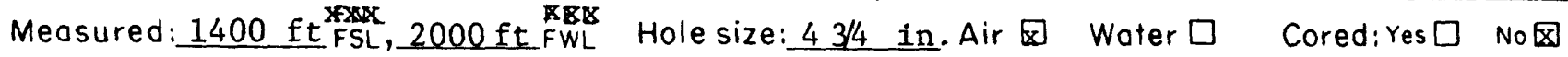
Remorks:

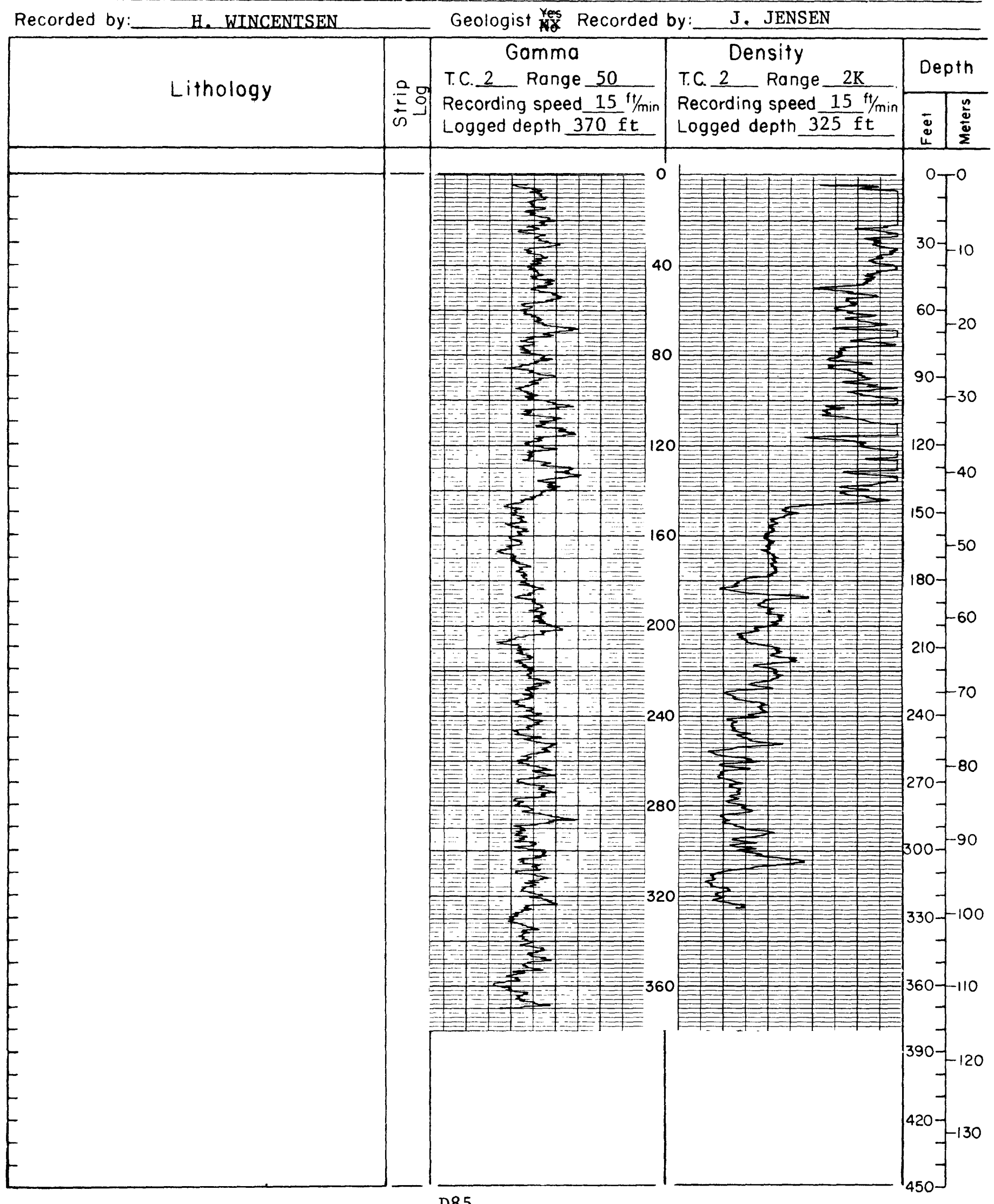


Montana College of Mineral Science and Technology

Hole no:US-77192 Map:Tree Coulee Schooloote1025/77 State Montana County: Garfield

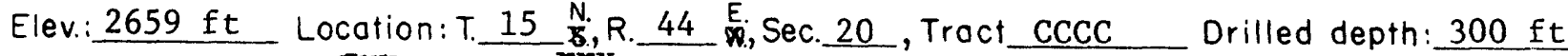

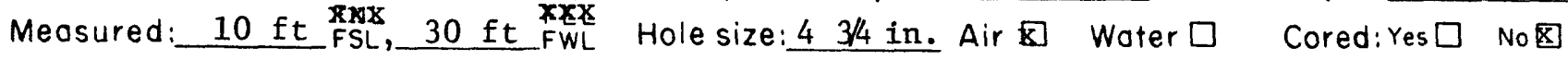
Remorks:

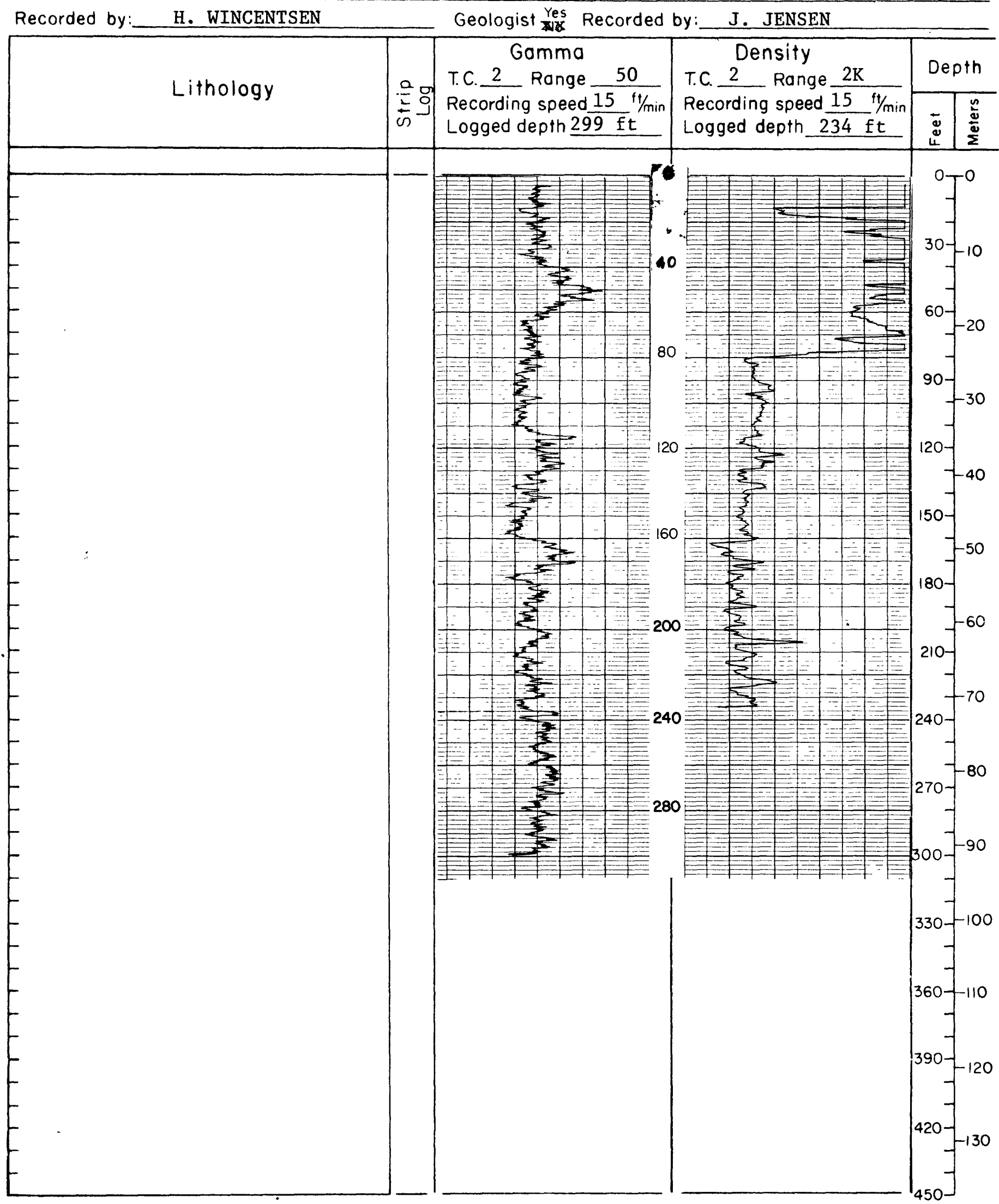


Montana College of Mineral Science and Technology

Hole no:US-77193 Map:Tree Coulee SchoolDote1026/77 State:Montana County: Garfield Elev: $2570 \mathrm{ft}$ Location:T. 16 N.R. 44 E. Sec. 30 , Troct_BABR Drilled depth: $300 \mathrm{ft}$

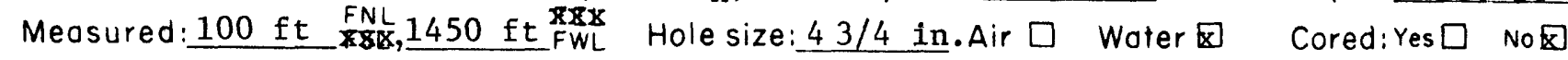

Remorks:

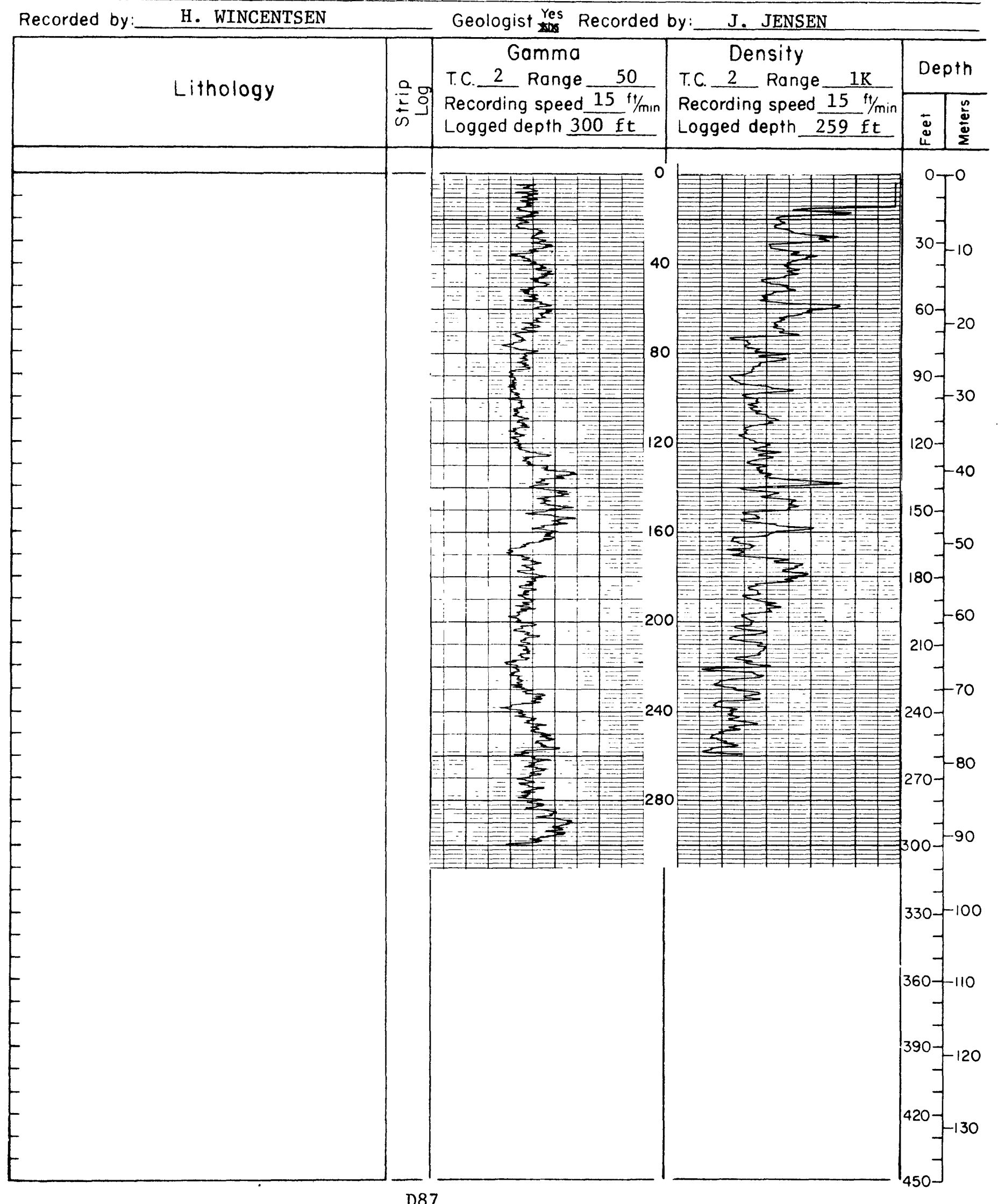


Montana College of Mineral Science and Technology

Hole no: US-77194 Mop:Hedstrom Lake SE Date:11/1/77 State:Montana County: Garfield Elev. $2768 \mathrm{fi}$ Location: T. $15 \mathrm{~N}, \mathrm{R} .44 \mathrm{E}$. Sec. 2 , Tract AAAA Drilled depth: $500 \mathrm{ft}$

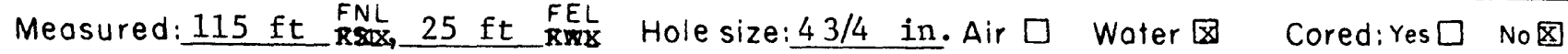

Remarks :

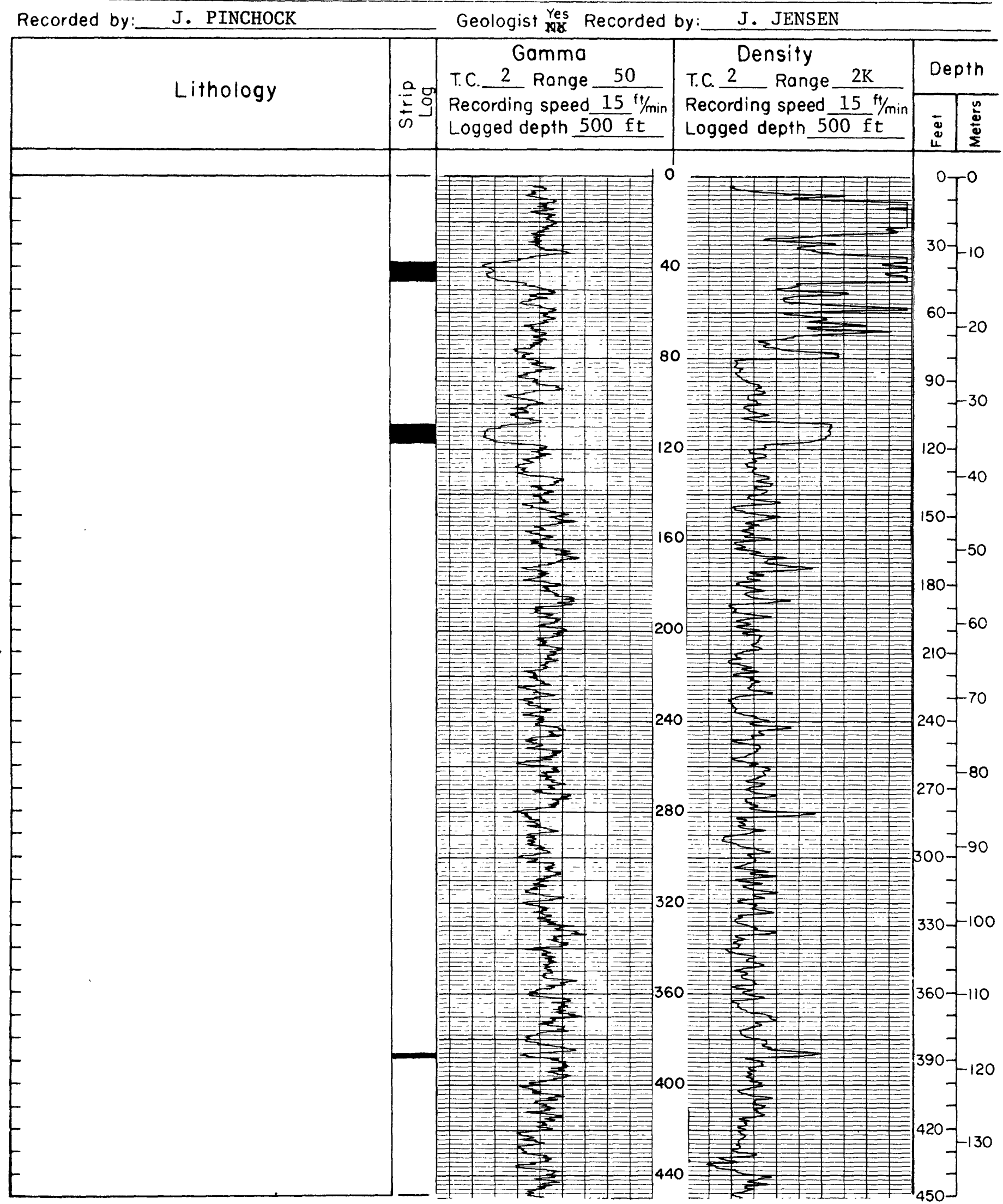


Hole number (continued)

$\underline{\text { US }-77194}$

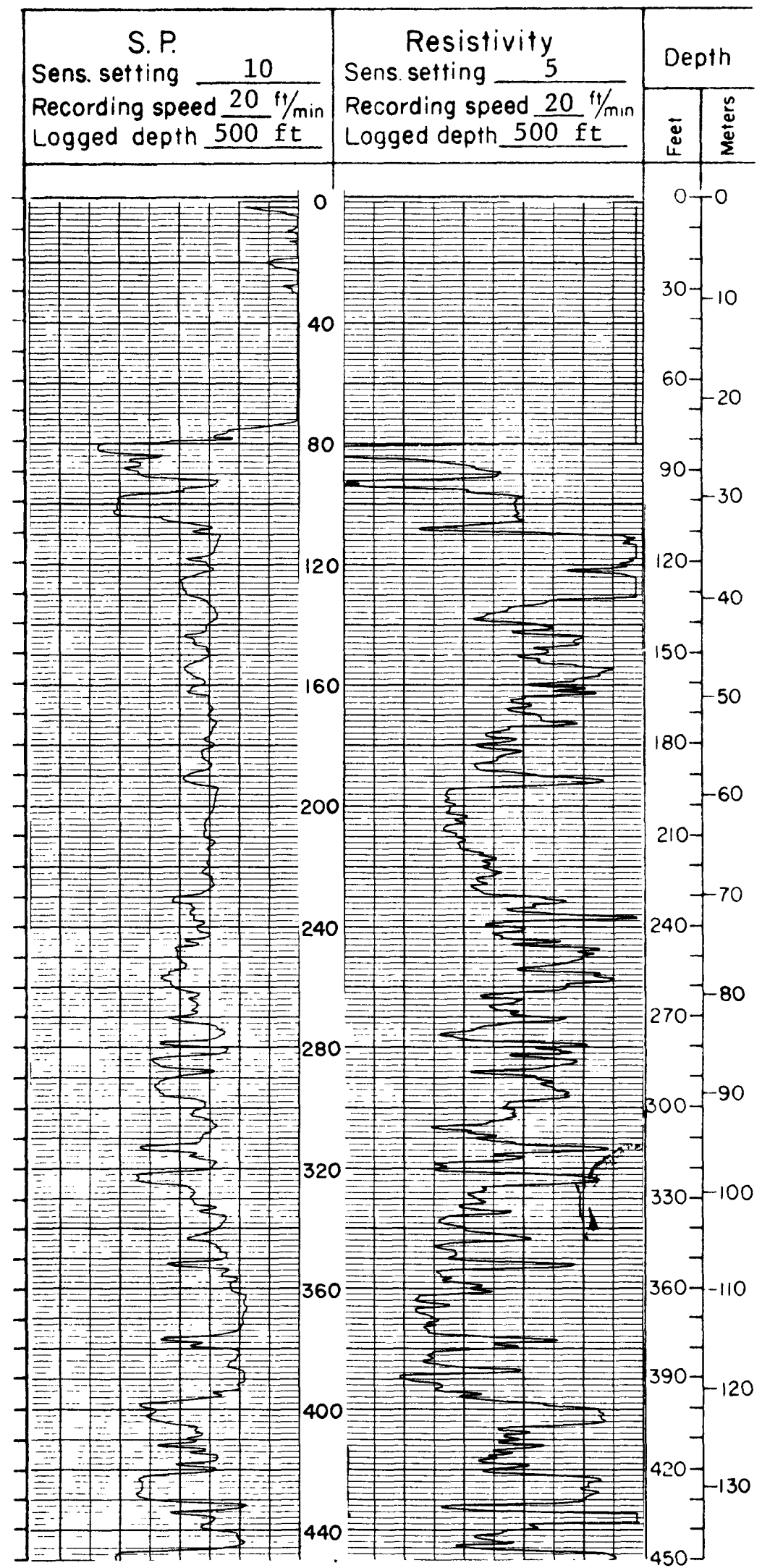


Hole number (continued)

US- 77194

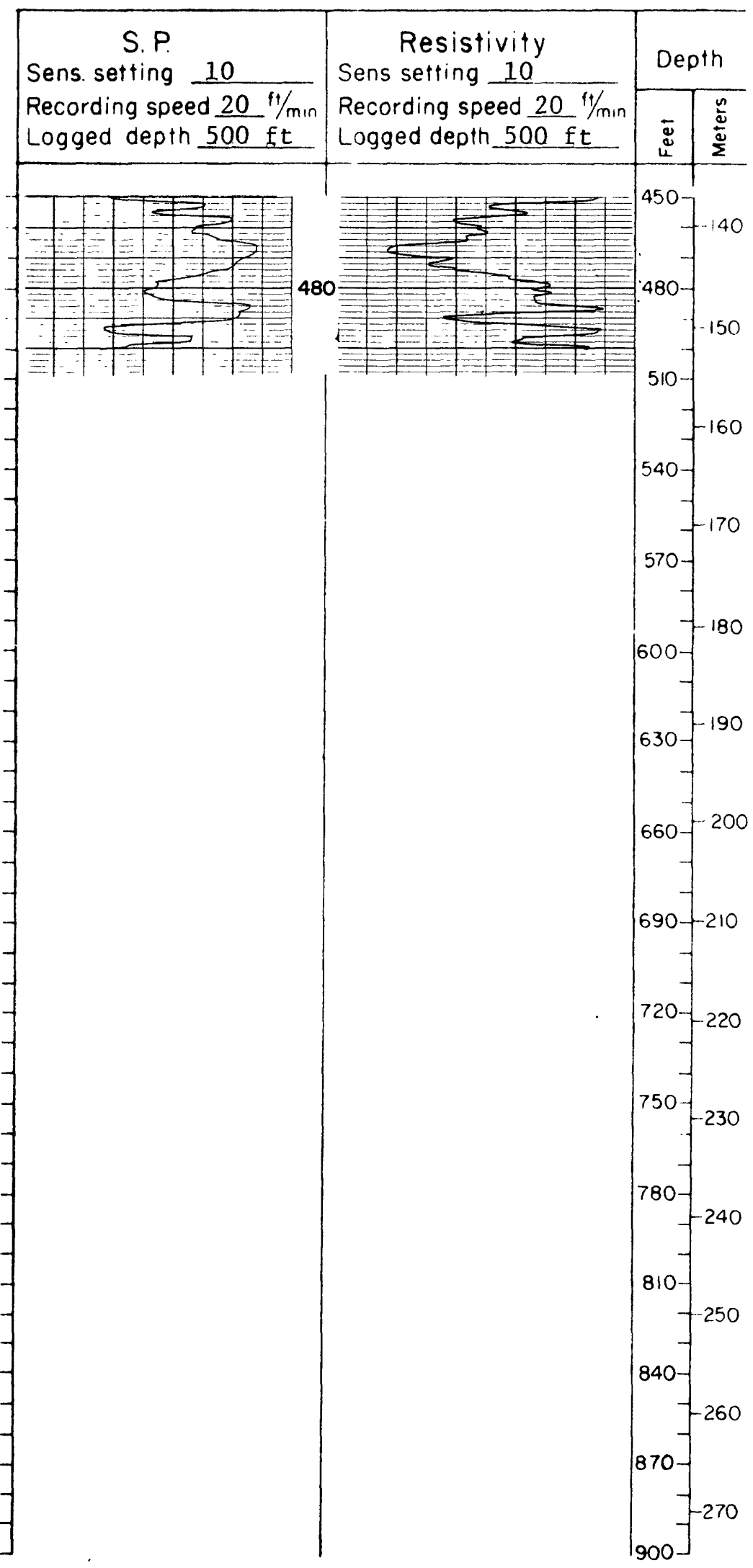

D91 
Hole no: US-77195 MopHedstrom Lake SE Date11/2/77 State:Montana County: Prairie Elev.:2783 ft Locotion:T. 16 N. R. 45 E. Sec. 34 , Tract AADD Drilled depth: $300 \mathrm{ft}$

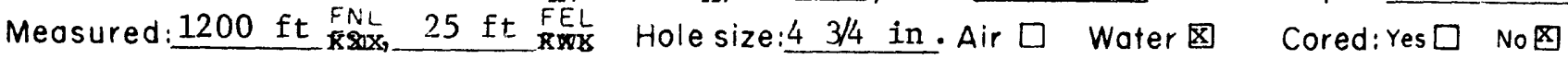
Remarks:

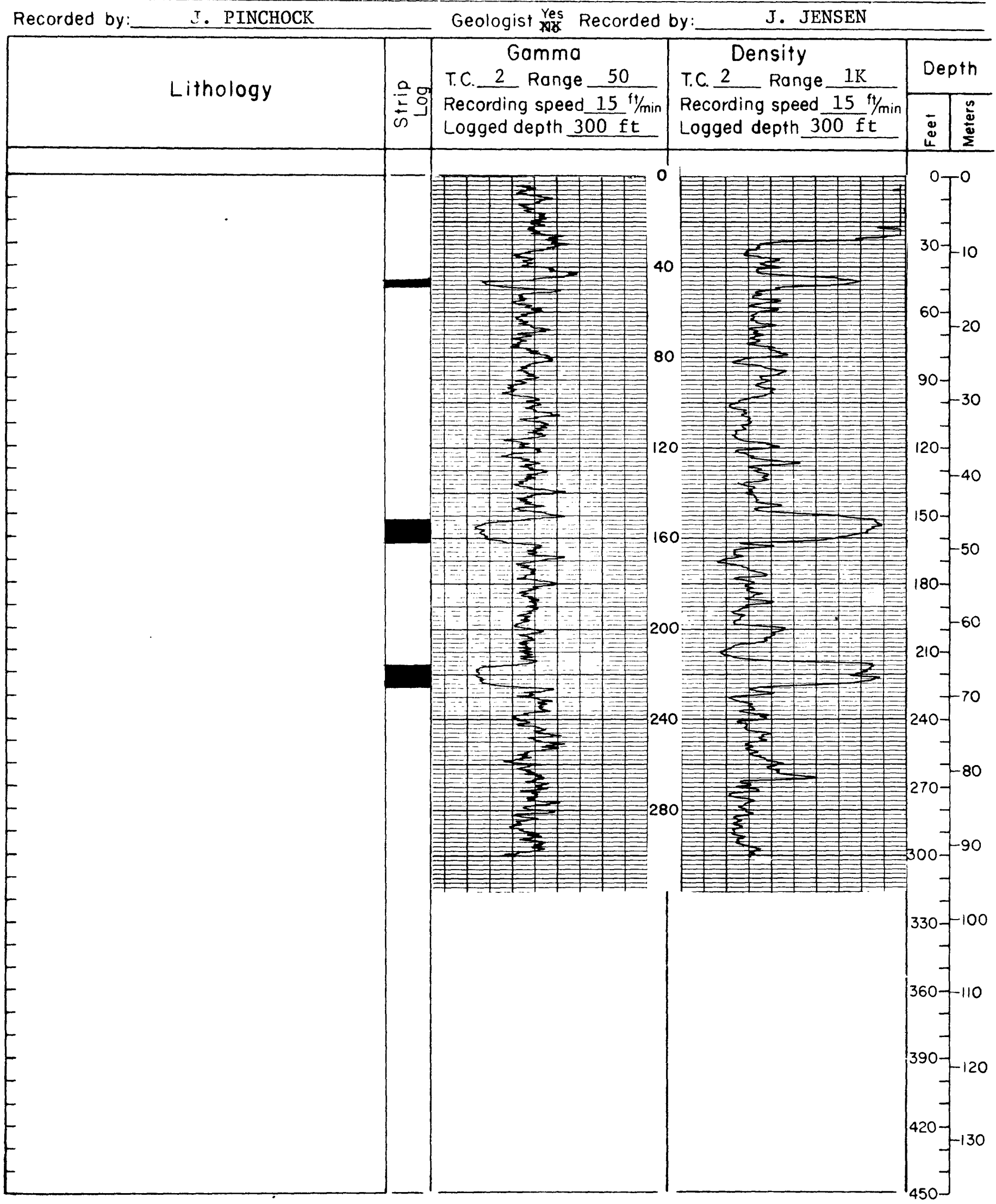


Hole number (continued)

$\underline{\text { US - } 77195}$

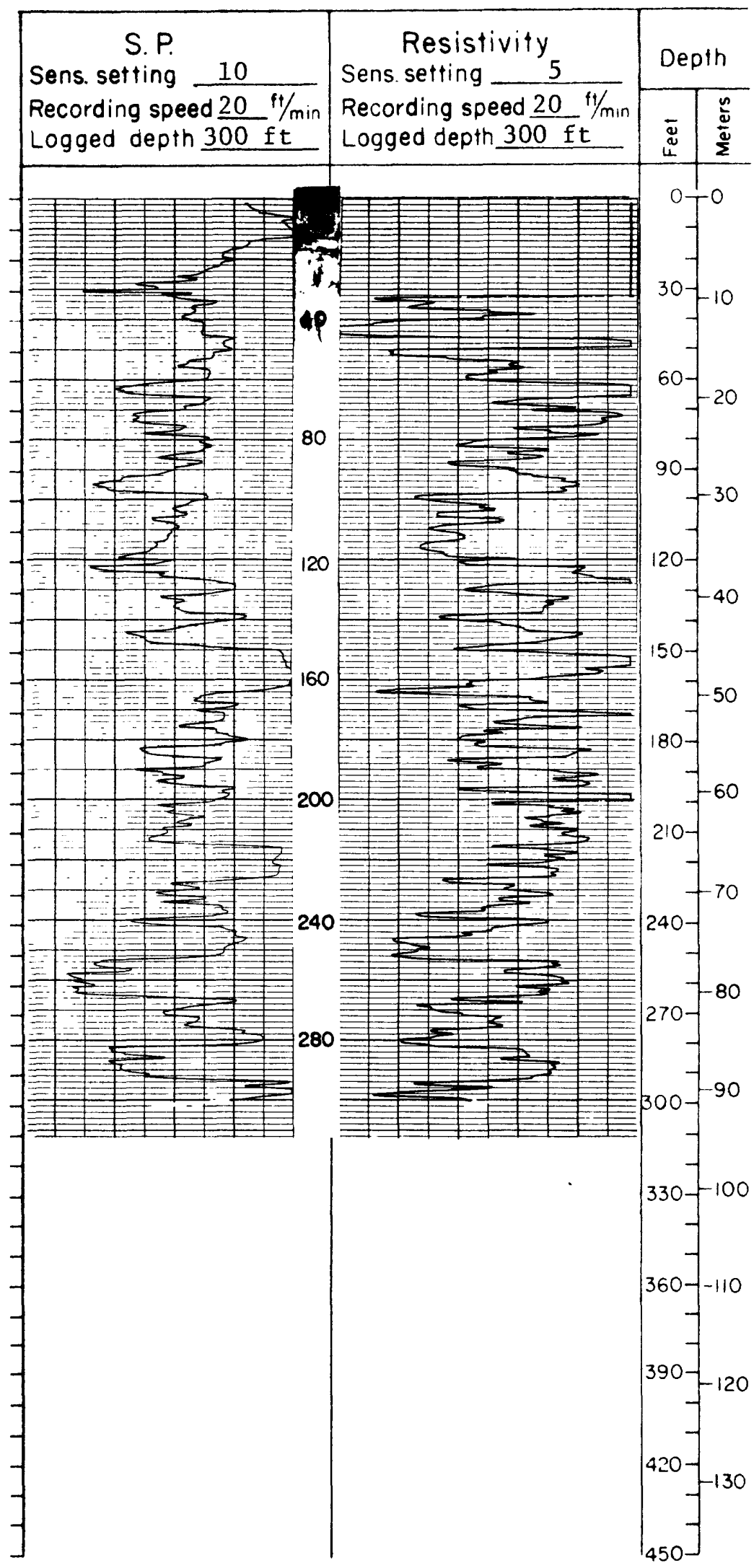


Hole no: US-77196 Map:Hedstrom Lake

Date:11/2/77 State: Montana County: Prairie Elev. $2782 \mathrm{ft}$ Locotion:T. 16 N. R. 45 E.,Sec. 8 , Tract_DCAD Drilled depth: $359 \mathrm{ft}$

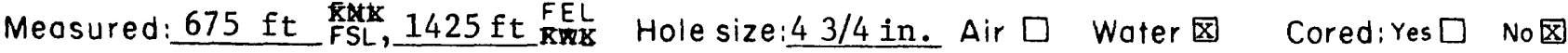

Remarks:

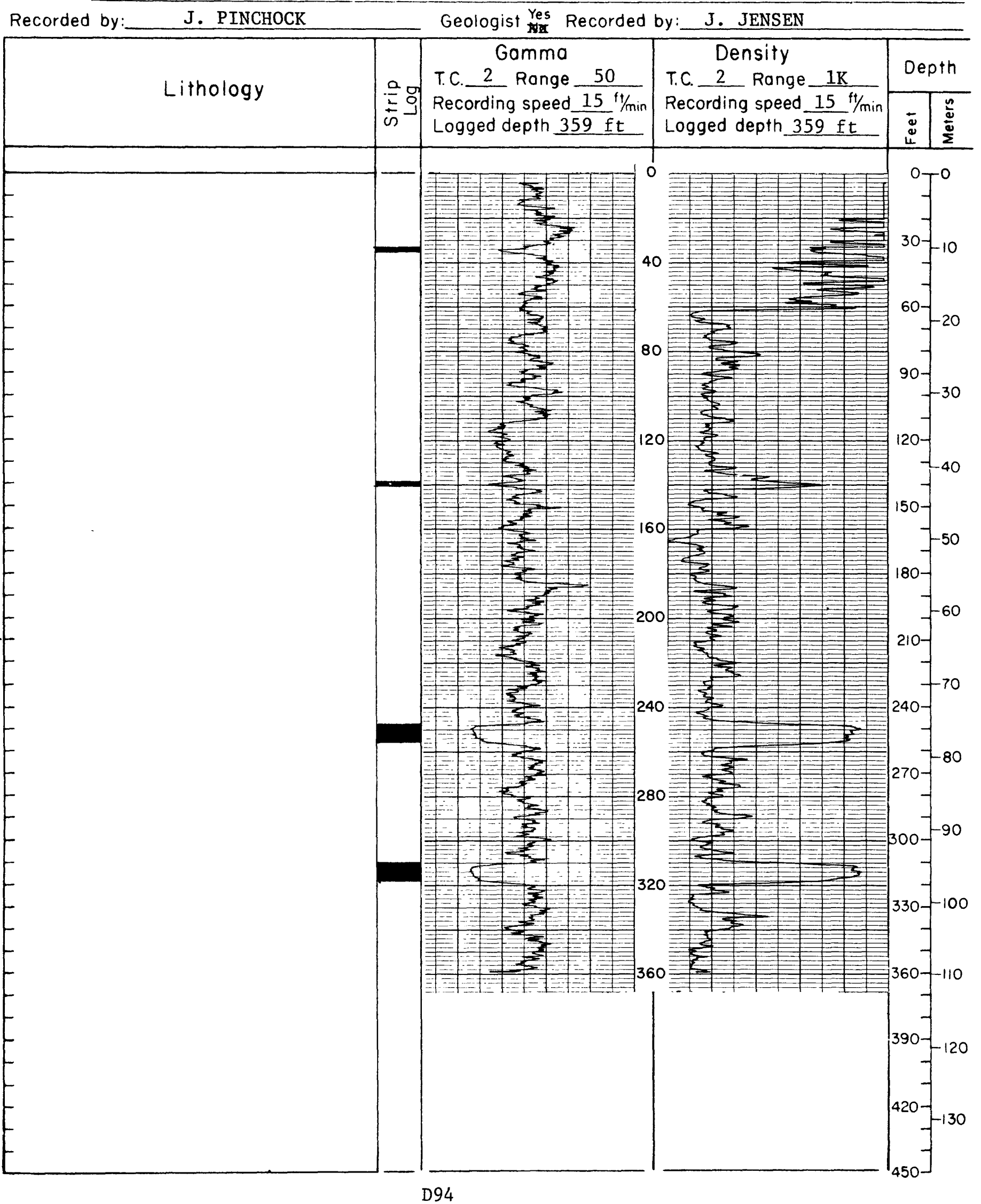


Hole number (continued)

$\underline{\text { US }-77196}$

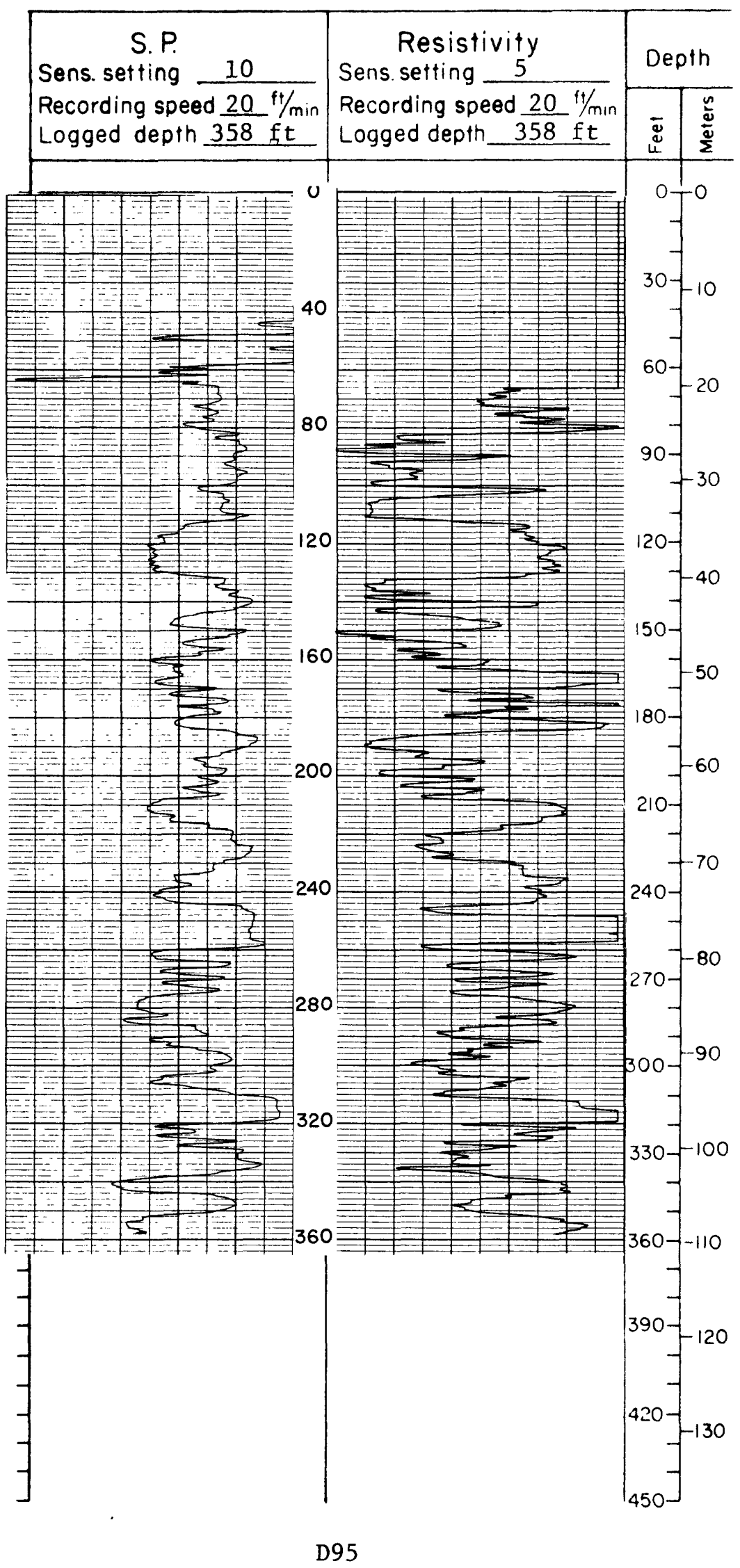


Montana College of Mineral Science and Technology

Hole no: US-77197 Map: Hedstrom Lake NW Dote:11/3/77 State: Montana County: Garfield Elev.: $2681 \mathrm{ft}$ Location: T. 16 N. R. $44 \mathrm{E}, \mathrm{Wec} .10$, Tract BBBB Drilled depth: $260 \mathrm{ft}$

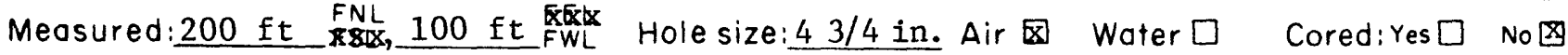
Remarks:

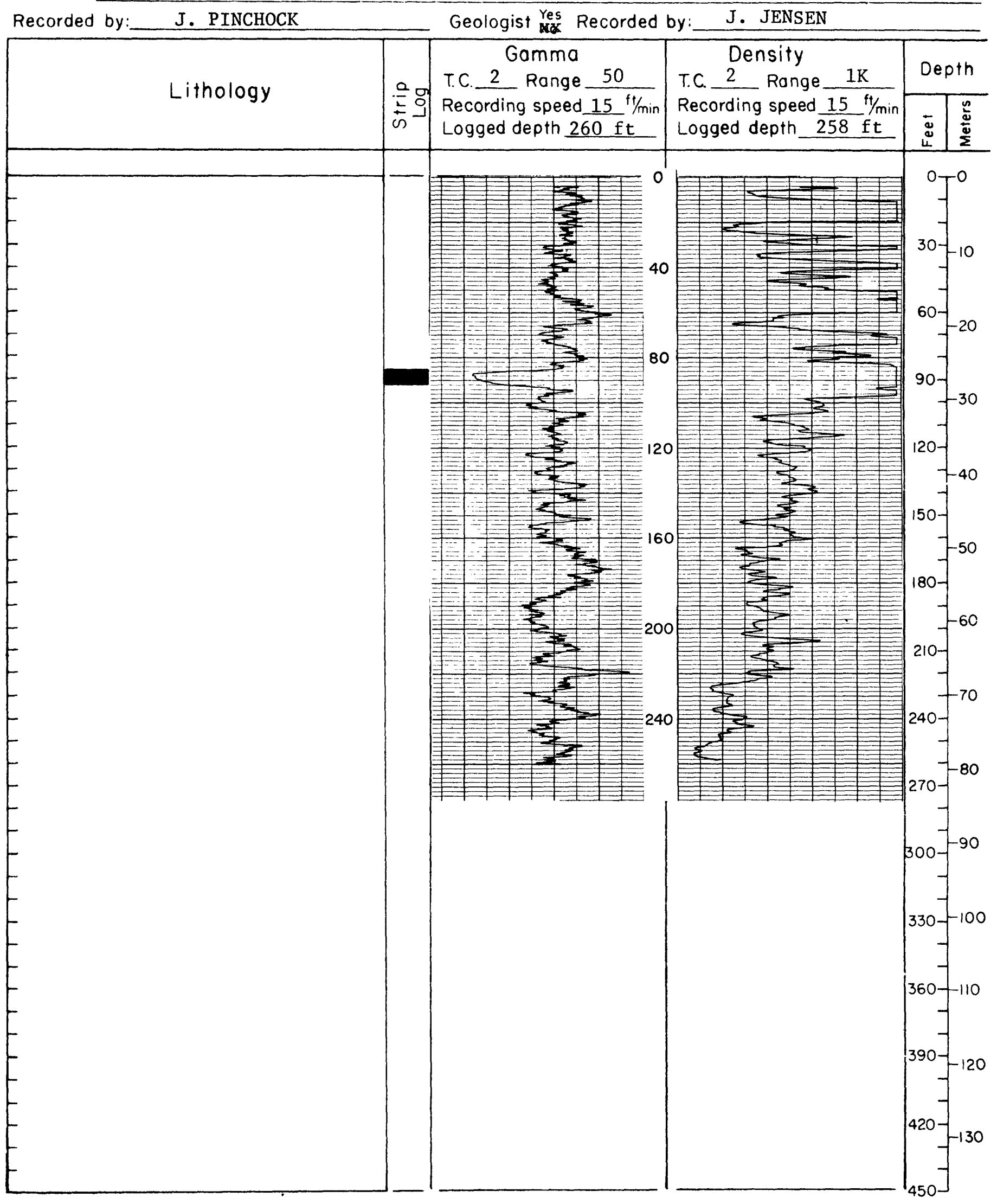


Montana College of Mineral Science and Technology

Hole no:US-77198 Map:Hedstrom Lake NW Dote:11/3/77 State: Montana County: McCone Elev.: $2561 \mathrm{ft}$ Location:T. $17 \mathrm{~N}, \mathrm{R} .44 \mathrm{E}$, Sec. 20 , Tract_BBBB Drilled depth: $200 \mathrm{ft}$ Measured: $80 \mathrm{ft}$ FNL, $5 \mathrm{ft}$ FWL Hole size:4 3/4 in. Air $\mathbf{F W}$ Water $\square$ Cored:Yes $\square$ No

Remorks:

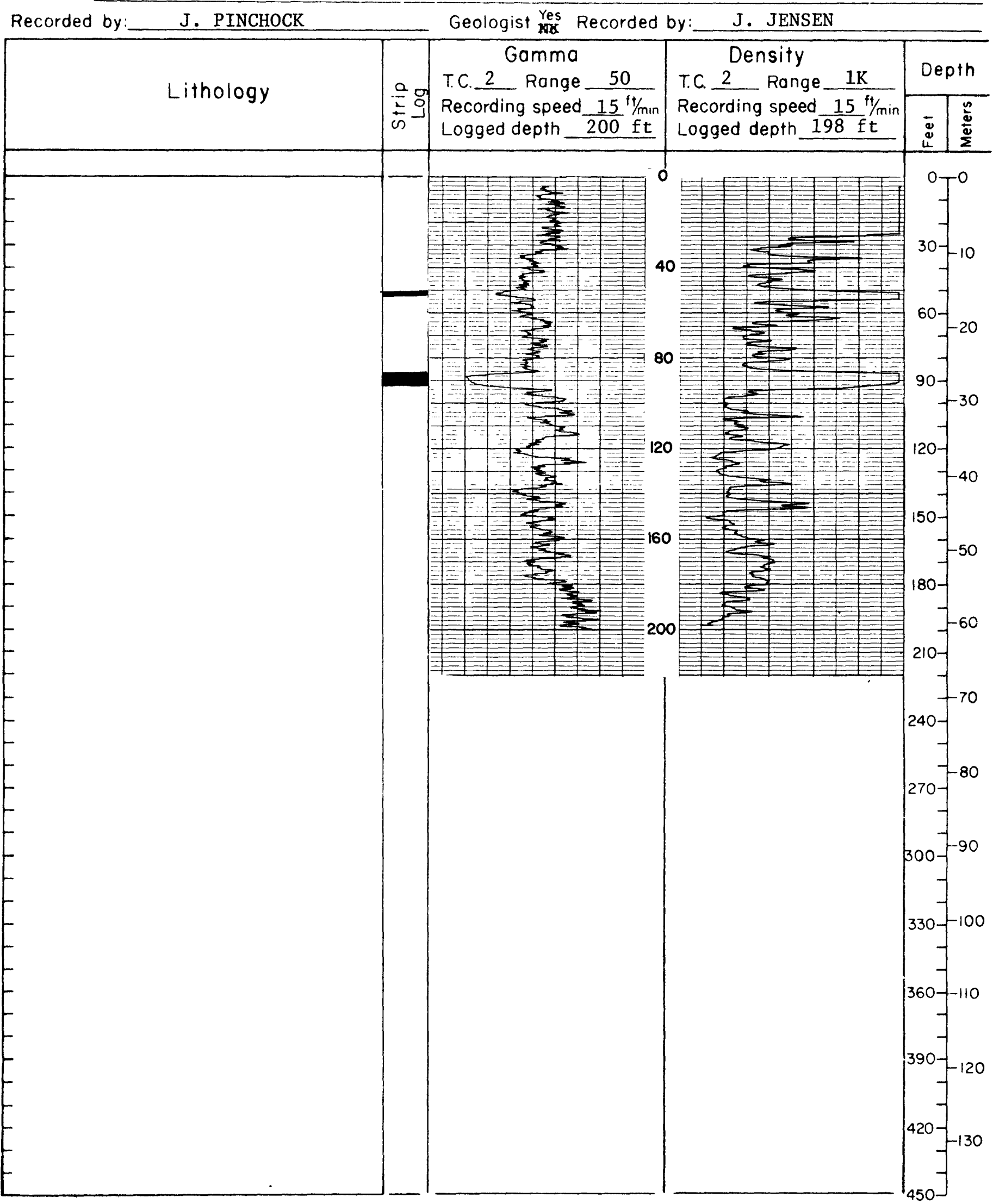


Montana College of Mineral Science and Technology

Hole no: IS -77199 Map: Berry School

Date:11/4/27 State:Montana County: McCone Elev. $2760 \mathrm{ft}$ Location: T._17 N., R. 45 W. Sec. 10 , Tract_ACCB Drilled depth: $480 \mathrm{ft}$

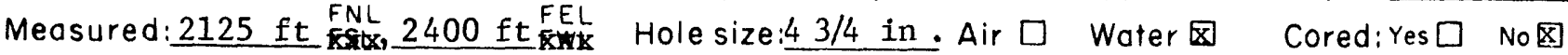

Remarks:

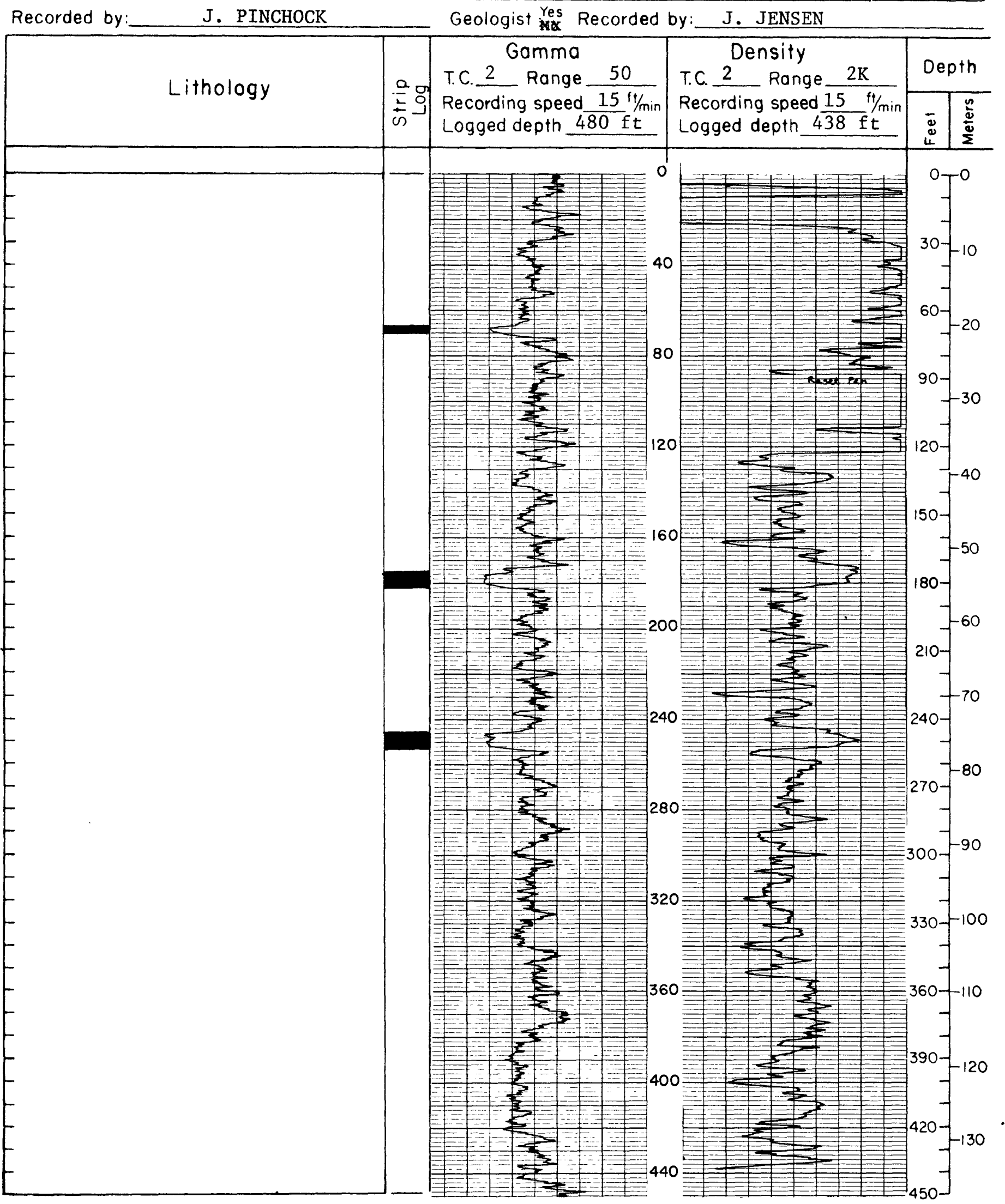


Hole number (continued)

US- 77199

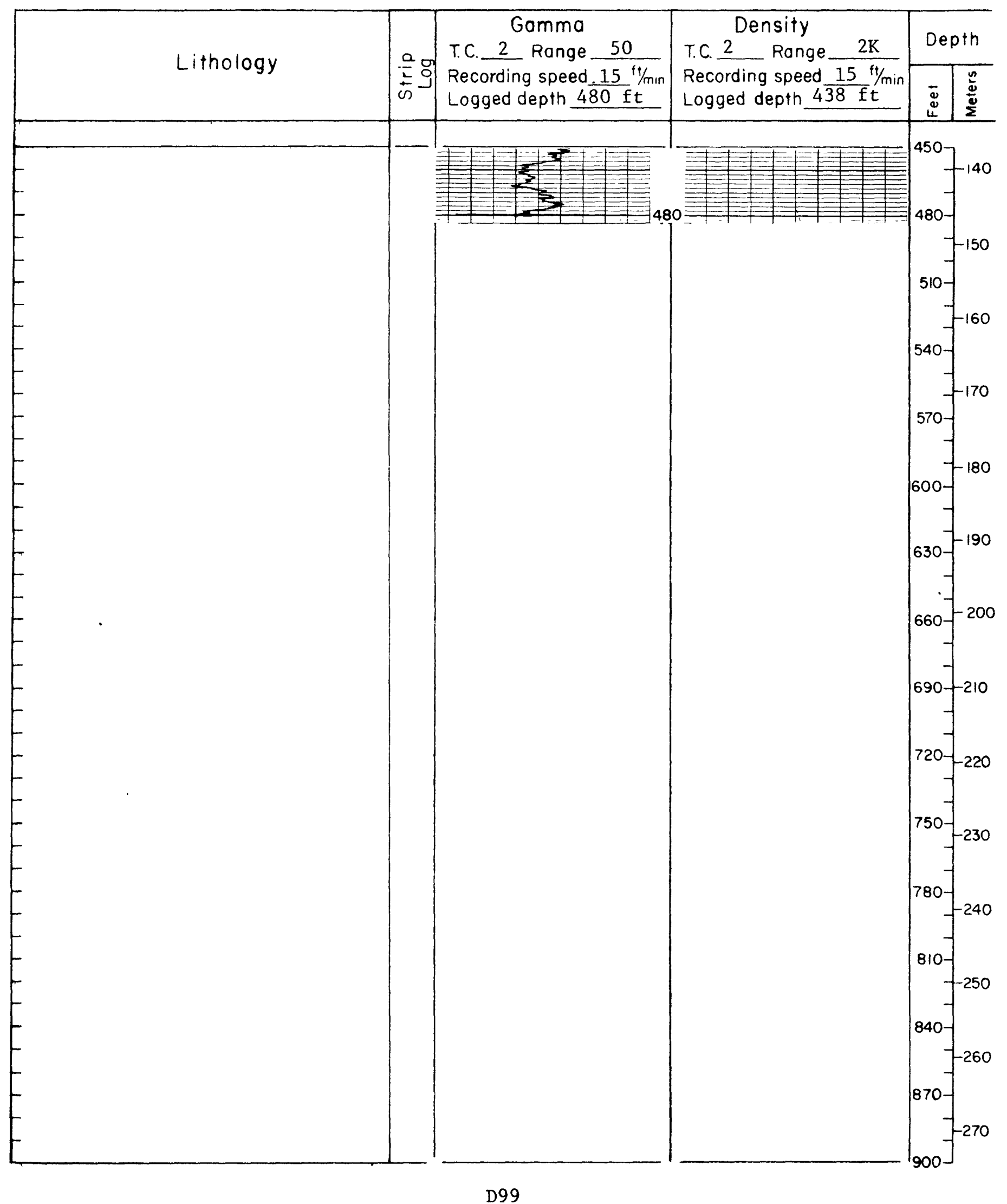




\section{References}

Kistner, F. B., 1977, Preliminary report of coal drill-hole data in the southern Powder River Basin, Converse County, Wyoming: U.S. Geol. Survey Open-File Report 77-774, 25 p., 1 fig.

U. S. Geological Survey and Montana Bureau of Mines and Geology, 1973, Preliminary report of coal drill-hole data and chemical analyses of coal beds in Sheridan and Campbell Counties, Wyoming; and Big Horn County, Montana: U.S. Geological Survey open-file report, $57 \mathrm{p}$.

1974, Preliminary report of coal dri11-hole data and chemical analyses of coal beds in Campbell County, Wyoming: U.S. Geol. Survey Open-File Report 74-97, 241 p., 1 fig., 3 tables. 1976a, Preliminary report of coal drillhole data and chemical analyses of coal beds in Campbell and Sheridan Counties, Wyoming; Custer, Prairie, and Garfield Counties, Montana; and Mercer County, North Dakota: U.S. Geol. Survey Open-File Report 76-319,. 377 p., 4 figs., 3 tables.

1976b, Preliminary report of coal drill-hole data and chemical analyses of coal beds in Campbe11, Converse, and Sheridan Counties, Wyoming; and Big Horn, Richland, and Dawson Counties, Montana: U.S. Geo1. Survey Open-File Report 76-450, 382 p., 4 figs. 1977a, Preliminary report of 1976 drilling of coals in Campbel1, and Sheridan Counties, Wyoming; and Big Horn, Dawson, McCone, Richland, Roosevelt, Rosebud, Sheridan and Wibaux Counties, Montana: U.S. Geological Survey Open-File Report 77-283, 403 P., 6 figs. 
U. S. Geological Survey and Montana Bureau of Mines and Geology, 1977b, Geophysical logs for Big Horn, Fallon, and Powder River Counties, Montana, chapter A of Preliminary report of 1977 coal drilling in eastern Montana and northeastern Wyoming: U.S. Geol. Survey Open-File Report 77-721-A, 77 p. 1977c, Geophysical logs for Big Horn County, Montana, chapter B of Preliminary report of 1977 coal drilling in eastern Montana and northeastern Wyoming: U.S. Geol. Survey Open-File Report 77-721-B, $126 \mathrm{p}$. 1978, Geophysical logs for Powder River and Dawson Counties, Montana, chapter C of Preliminary report of 1977 coal drilling in eastern Montana and northeastern Wyoming: U.S. Geol. Survey Open-File Report 77-721-C, $79 \mathrm{p}$.

U. S. Geological Survey and North Dakota Geological Survey, 1976, Preliminary report on 1975 drilling of lignites in western North $\mathrm{Da}-$ kota: Adams, Bowman, Dunn, Hettinger, McLean, Mercer, Oliver, Slope, and Williams Counties: U.S. Geol. Survey Open-File Report $76-869,144$ p., 5 figs. 1977, Preliminary report on 1976 lignite drilling in western North Dakota: Adams, Billings, Dunn, Hettinger, McKenzie, Mercer, Morton, 01iver, Slope, and Stark Counties: U.S. Geol. Survey Open-File Report 77-857, 336 p., 5 figs. 